REPRESENTATION THEORY

An Electronic Journal of the American Mathematical Society

Volume 3, Pages 58-89 (June 9, 1999)

S $1088-4165(99) 00071-0$

\title{
SQUARE INTEGRABLE REPRESENTATIONS OF CLASSICAL $p$-ADIC GROUPS CORRESPONDING TO SEGMENTS
}

\author{
MARKO TADIĆ
}

\begin{abstract}
Let $S_{n}$ be either the group $S p(n)$ or $S O(2 n+1)$ over a $p$-adic field $F$. Then Levi factors of maximal parabolic subgroups are (isomorphic to) direct products of $G L(k)$ and $S_{n-k}$, with $1 \leq k \leq n$. The square integrable representations which we define and study in this paper (and prove their square integrability), are subquotients of reducible representations $\operatorname{Ind}_{P}^{S_{n}}(\delta \otimes \sigma)$, where $\delta$ is an essentially square integrable representation of $G L(k)$, and $\sigma$ is a cuspidal representation of $S_{n-k}$. These square integrable representations play an important role in a construction of more general square integrable representations.
\end{abstract}

\section{INTRODUCTION}

The problem of classification of noncuspidal irreducible square integrable representations of a reductive group $G$ over a $p$-adic field $F$, is equivalent to the problem of classification of irreducible square integrable subquotients of the representations parabolically induced from irreducible cuspidal representations of proper Levi subgroups. We shall denote the classical group $S p(n, F)$ or $S O(2 n+1, F)$ by $S_{n}$, and we shall fix one of these series of groups (we shall assume $\operatorname{char}(F) \neq 2$ ). Since the Levi factors of the maximal parabolic subgroups of $S_{n}$ are naturally isomorphic to $G L(k, F) \times S_{n-k}$, we shall denote by $\pi \rtimes \sigma$ the representation of $S_{n}$ parabolically induced from $\pi \otimes \sigma\left(\pi\right.$ and $\sigma$ are admissible representations of $G L(k, F)$ and $S_{n-k}$ respectively; the precise definition of $\rtimes$ can be found in Section 1$)$. This definition is a natural generalization of the multiplication $\times$ between representations of general linear groups introduced by J. Bernstein and A.V. Zelevinsky. Using this notation, we can say that for the classification of noncuspidal irreducible square integrable representations of the groups $S_{n}$, one needs to classify such subquotients of

$$
\rho_{1} \times \rho_{2} \times \cdots \times \rho_{m} \rtimes \sigma,
$$

where $\rho_{i}$ are irreducible cuspidal representations of general linear groups, and $\sigma$ is a similar representation of some $S_{k}$.

For the first case of $m=1$, one only needs to know the reducibility of $\rho \rtimes \sigma$ (such reducibility will be called the cuspidal or generalized rank one reducibility). Let us recall that each irreducible essentially square integrable representation $\delta$ of a general linear group can be written as $|\operatorname{det}|_{F}^{e(\delta)} \delta^{u}$, where $e(\delta) \in \mathbb{R}$ and $\delta^{u}$ is a unitarizable representation $\left(||_{F}\right.$ is the modulus character of $\left.F\right)$. Now look at the simplest example in the case of $m=1$, when $\rho$ is a character and $\sigma$ is trivial. Then,

Received by the editors July 17, 1998 and, in revised form, December 6, 1998.

1991 Mathematics Subject Classification. Primary 22E50.

(C)1999 American Mathematical Society 
it is well known from the representation theory of $S L(2, F)$ and $S O(3, F)$, that in the case of reducibility we have

$$
e(\rho) \in\{0, \pm 1 / 2, \pm 1\}
$$

F. Shahidi has proved that this is the case in general, if $\sigma$ is generic ([Sh1]). In general, cuspidal reducibility in $\{0, \pm 1 / 2, \pm 1\}$ will be called generic or nonexceptional reducibility. Otherwise, we shall say that we have an exceptional or nongeneric reducibility. The existence of exceptional reducibilities was proved recently by M. Reeder and by C. Mœglin. One can expect that any cuspidal reducibility always lies in $(1 / 2) \mathbb{Z}$ (Conjecture 9.4 of [Sh1] implies this).

The square integrable representations which we define in this paper (and prove their square integrability) are subquotients of reducible representations $\delta \rtimes \sigma$, where $\delta$ is an essentially square integrable representation of a general linear group, and $\sigma$ is a cuspidal representation of some $S_{k}$. This construction is a part of a wider strategy of construction of noncuspidal irreducible square integrable representations of the groups $S_{n}$. Before we describe briefly this strategy, we shall introduce some notation. Denote $|\operatorname{det}|_{F}$ by $\nu$. A segment in irreducible cuspidal representations of general linear groups is a set $\Delta=\left\{\rho, \nu \rho, \ldots, \nu^{k} \rho\right\}$, where $\rho$ is an irreducible cuspidal representation attached to a general linear group. Let $\tilde{\Delta}=\{\tilde{\pi} ; \pi \in \Delta\}$, where $\tilde{\pi}$ denotes the contragredient representation of $\pi$. For such a segment $\Delta$, the representation $\nu^{k} \rho \times \nu^{k-1} \rho \times \cdots \times \nu \rho \times \rho$ has a unique irreducible essentially square integrable subquotient (it is a unique irreducible subrepresentation), which we denote by $\delta(\Delta)$. The first part of our strategy of construction of noncuspidal irreducible square integrable representations of the groups $S_{n}$ is to consider segments $\Delta$ of irreducible cuspidal representations of general linear groups satisfying that $\delta(\Delta) \rtimes \sigma$ reduces and, if $\Delta \cap \tilde{\Delta} \neq \emptyset$, then $\delta(\Delta \cap \tilde{\Delta}) \rtimes \sigma$ also reduces. Then to each such segment, one attaches irreducible square integrable representations (closely related to it). In the case when $\Delta \cap \tilde{\Delta}=\emptyset$, we have in [T4] such a segment attached to an irreducible square integrable representation (the simplest example of such representations are the Steinberg representations).

In this paper we attach two irreducible square integrable representations to each segment as above, for which $\Delta \cap \tilde{\Delta} \neq \emptyset$. The construction in this case is significantly more complicated than in the case $\Delta \cap \tilde{\Delta}=\emptyset$, since in this case we need to work with highly nonregular representations.

The second step in our strategy would be attaching to a sequences of segments as above (satisfying certain additional conditions among them) families of irreducible square integrable representations, using the above square integrable representations attached to single segments. Such a type of construction can be found in [T6]. G. Muić has shown in [Mi2] that the family constructed in [T6] contains all the generic irreducible square integrable representations of the groups $S_{n}$ (they make a relatively small part of the whole family).

In other words, we expect that the representations that we construct in this paper are part of basic building blocks of general square integrable representations of the groups $S_{n}$.

Now we shall describe the main result of the paper.

Theorem. Let $\rho$ and $\sigma$ be irreducible unitarizable cuspidal representations of $G L(p, F)$ and $S_{q}$ respectively, such that there exists $\alpha \in(1 / 2) \mathbb{Z}, \alpha \geq 0$ satisfying that $\nu^{\alpha} \rho \rtimes \sigma$ reduces and $\nu^{\beta} \rho \rtimes \sigma$ is irreducible for $\beta \in(\alpha+\mathbb{Z}) \backslash\{ \pm \alpha\}$. Let $\Delta$ be 
a segment in cuspidal representations of general linear groups such that $e(\delta(\Delta))>0$ and $\nu^{\alpha} \rho \in \Delta \cap \tilde{\Delta}$. Then:

(i) The representation $\delta(\Delta) \rtimes \sigma$ has exactly two irreducible subrepresentations. They are square integrable and each of them has multiplicity one in $\delta(\Delta) \rtimes \sigma$.

(ii) The representation $\delta(\Delta \cap \tilde{\Delta}) \rtimes \sigma$ reduces into a sum of two inequivalent irreducible tempered subrepresentations $\tau_{1}$ and $\tau_{2}$. The representation $\delta(\Delta \backslash \tilde{\Delta}) \rtimes \tau_{i}$ has a unique irreducible subrepresentation, which we denote by $\delta(\Delta, \sigma)_{\tau_{i}}$. The representations $\delta(\Delta, \sigma)_{\tau_{i}}, i=1,2$ are irreducible subrepresentations of $\delta(\Delta) \rtimes \sigma$.

Suppose $\operatorname{char}(F)=0$ and that Conjecture 9.4 of [Sh1] holds. Then the assumption of the theorem on $\Delta$ and $\sigma$ has the very simple form: $e(\delta(\Delta))>0$ and $\delta(\Delta \cap \tilde{\Delta}) \rtimes \sigma$ reduces (which implies that $\delta(\Delta) \rtimes \sigma$ reduces).

Our methods in this paper are based on techniques of Jacquet modules, the structure related to them ([T3]) and systematic use of the Bernstein-Zelevinsky theory $([\mathrm{Z}])$. In this paper, besides the proof of the square integrability of the representations $\delta(\Delta, \sigma)_{\tau}$, we get also, rather explicit information about some of their Jacquet models (see Theorems 4.5, 4.7 and 5.5; for tempered representations see Theorems 2.3 and 2.5). The paper would be considerably shorter, if we restricted ourselves to the proof of the square integrability only. The heart of the paper is the second, third and fourth sections. Section 5 is included, because in the case of unitary reducibility, some technical modifications are necessary (although this section follows the ideas of Section 4).

Now we shall describe the content of the sections. In Section 1 we introduce the notation and recall of some results that we shall need in the remaining sections. Section 2 studies the tempered representations which are crucial for our approach to the square integrable representations. In Section 3 we define the square integrable representations $\delta(\Delta, \sigma)_{\tau}$ and observe some of their basic properties. In Sections 4 and 5 we prove their square integrability. Section 6 describes an example which shows that, besides the reducibility of $\delta(\Delta) \rtimes \sigma$, the reducibility of $\delta(\Delta \cap \tilde{\Delta}) \rtimes \sigma$ is also important for appearance of square integrable representations (this assumption also seems to be natural from the point of the conjectural local Langlands' correspondence). In Section 7, we write a proof of a simple fact from the representation theory of general linear groups, for which we did not know a reference.

Let us note that several years ago we had a proof of the above theorem in the case of generic reducibility at $0,1 / 2$ and 1 (at that time, we did not have any evidence that nongeneric reducibilities can actually show up). Technical details of the proofs in that approach were very much dependent on the specific value of reducibility (although the ideas of the proofs were the same). Our present approach, which covers reducibilities $\alpha \in(1 / 2) \mathbb{Z}, \alpha \geq 0$, is almost independent of the value of the reducibility (only the reducibility at 0 requires some separate attention).

We want to thank the referee for a number of useful suggestions and corrections.

\section{Notation}

In this paper, we fix a $p$-adic field $F, \operatorname{char}(F) \neq 2$. For a reductive group $G$ over $F$, there is a natural map from the smooth representations of finite length into the Grothendieck group $\mathfrak{R}(G)$ of the category of all smooth representations of $G$ of finite length. This map will be denoted by s.s. and called semisimplification. Further, there is a natural partial order $\leq$ on $\mathfrak{R}(G)$ (the cone $\{\varphi \in \mathfrak{R}(G) ; \varphi \geq 0\}$ consists of 
the semisimplifications of all smooth representations of $G$ of finite length). Denote by $\tilde{G}$ the set of equivalence classes of all irreducible smooth representations of $G$. The group $\mathfrak{R}(G)$ can be identified with the free $\mathbb{Z}$-module which has $\tilde{G}$ for a basis (in this way, we shall consider $\tilde{G} \subseteq \mathfrak{R}(G)$ ). Then for $\varphi=\sum_{\pi \in \tilde{G}} n_{\pi} \pi \in \mathfrak{R}(G), \varphi \geq 0$ if and only if $n_{\pi} \geq 0$ for all $\pi \in \tilde{G}$. For two finite length representations $\pi_{1}$ and $\pi_{2}$ of $G, \pi_{1} \leq \pi_{2}$ will denote s.s. $\left(\pi_{1}\right) \leq$ s.s. $\left(\pi_{2}\right)$ (i.e., the inequality between their semi simplifications). The inequality $\pi_{1} \leq \pi_{2}$ is equivalent to the fact that for each irreducible smooth representation $\tau$ of $G$ the multiplicity of $\tau$ in $\pi_{1}$ is less than or equal to the multiplicity of $\tau$ in $\pi_{2}$. Note that the functors of parabolic induction and the Jacquet functors respect these orders.

For a family of reductive groups $G_{i}$, the natural orders on $\mathfrak{R}\left(G_{i}\right)$ induce a natural order on the sum $\bigoplus_{i} \mathfrak{R}\left(G_{i}\right)$, which we shall denote by $\leq$ again. Let $\varphi \in \bigoplus_{i} \mathfrak{R}\left(G_{i}\right)$, let $\tau$ be an irreducible smooth representation of some $G_{i}$ and let $k \in \mathbb{Z}$. Suppose $\varphi \geq 0$. We shall say that the multiplicity of $\tau$ in $\varphi$ is $k$ if $k \tau \leq \varphi$ and $(k+1) \tau \not \leq \varphi$ (then $k \geq 0$ ).

In this paper, for a reductive group $G$ we shall always fix the minimal parabolic subgroup $P_{\emptyset}=M_{\emptyset} N_{\emptyset}$ consisting of all upper triangular matrices in $G$ (these subgroups will be minimal parabolic subgroups for the groups considered in this paper). The standard parabolic subgroups will be those which contain $P_{\emptyset}$.

Now we shall recall the notation for general linear groups. For a partition $\alpha=\left(n_{1}, \ldots, n_{k}\right)$ of $n$, we denote by $M_{\alpha}^{G L}=\left\{\mathrm{q}-\operatorname{diag}\left(g_{1}, \ldots, g_{k}\right) ; g_{i} \in G L\left(n_{i}, F\right)\right\}$, and $P_{\alpha}^{G L}=M_{\alpha}^{G L} N_{\emptyset}^{G L}$, where $N_{\emptyset}^{G L}$ denotes the subgroup of all upper triangular unipotent representations in $G L(n, F)$ and q-diag $\left(g_{1}, \ldots, g_{k}\right)$ denotes a quasidiagonal matrix having matrices $g_{1}, \ldots, g_{k}$ on the quasidiagonal.

For admissible representations $\pi_{1}$ and $\pi_{2}$ of $G L\left(n_{1}, F\right)$ and $G L\left(n_{2}, F\right)$ respectively, one can consider $\pi_{1} \otimes \pi_{2}$ as a representation of $G L\left(n_{1}, F\right) \times G L\left(n_{2}, F\right) \cong$

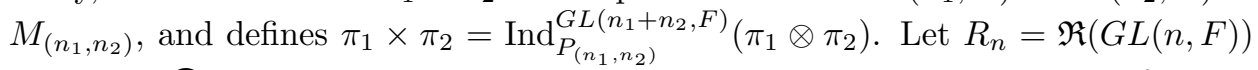
and $R=\bigoplus_{n \geq 0} R_{n}$. The above operation $\times$ between representations, lifts in a natural way to a mapping $\times: R_{n_{1}} \times R_{n_{2}} \rightarrow R_{n_{1}+n_{2}}$, and further to $\times: R \times R \rightarrow R$ (for details see [Z]). In this way, $R$ becomes a graded ring. Let $m, s: R \times R \rightarrow R$ be maps defined by $m\left(\sum_{i} x_{i} \otimes y_{i}\right)=\sum_{i} x_{i} \times y_{i}$ and $s\left(\sum_{i} x_{i} \otimes y_{i}\right)=\sum_{i} y_{i} \otimes x_{i}$. The additive map of $R$ which sends irreducible representations to its contragredients will be denoted by $\sim: R \rightarrow R$.

For an irreducible admissible representation $\pi$ of $G L(n, F)$, denote by $m^{*}(\pi)$ the sum of semisimplifications of normalized Jacquet modules of $\pi$ with respect to parabolic subgroups $P_{(k, n-k)}^{G L}, k \in\{0,1, \ldots, n\}$. Then one can consider $m^{*}(\pi)$ as an element of $R \otimes R$. Lifting $m^{*}$ to an additive map $m^{*}: R \rightarrow R \otimes R$, one gets a structure of a graded Hopf algebra on $R$.

Let $\nu=|\operatorname{det}|_{F}: G L(n, F) \rightarrow \mathbb{R}^{\times}$, where ||$_{F}$ denotes the modulus character of $F$. Each irreducible essentially square integrable representation $\delta$ of a general linear group can be written $\delta=\nu^{e(\delta)} \delta^{u}$, where $e(\delta) \in \mathbb{R}$ and $\delta^{u}$ is unitarizable. For an irreducible cuspidal representation of a general linear group, the set $\left[\rho, \nu^{m} \rho\right]=\left\{\rho, \nu \rho, \ldots, \nu^{m} \rho\right\}$ is called a segment in irreducible cuspidal representations of general linear groups. The representation $\nu^{m} \rho \times \nu^{m-1} \rho \times \cdots \times \nu \rho \times \rho$ contains a unique irreducible subrepresentation which we denote by $\delta\left(\left[\rho, \nu^{m} \rho\right]\right)$. 
This subrepresentation is essentially square integrable and

$$
m^{*}\left(\delta\left(\left[\rho, \nu^{m} \rho\right]\right)\right)=\sum_{k=-1}^{m} \delta\left(\left[\nu^{k+1} \rho, \nu^{m} \rho\right]\right) \otimes \delta\left(\left[\rho, \nu^{k} \rho\right]\right) .
$$

Here (and also in the sequel), we shall take $\left[\nu^{k} \rho, \nu^{k^{\prime}} \rho\right]=\emptyset$ if $k>k^{\prime}$, while $\delta(\emptyset)$ will denote the identity of $R$.

For a sequence $\delta_{1}, \ldots, \delta_{k}$ of irreducible essentially square integrable representations of general linear groups, take a permutation $p$ of $\{1, \ldots, k\}$ such that $e\left(\delta_{p(1)}\right) \geq\left(\delta_{p(2)}\right) \geq \cdots \geq e\left(\delta_{p(k)}\right)$. Then the representation $\delta_{p(1)} \times \delta_{p(2)} \times \cdots \times \delta_{p(k)}$ has a unique irreducible quotient, which we shall denote by $L\left(\delta_{1}, \ldots, \delta_{k}\right)$. We shall consider a partial order $\leq$ defined in 7.1 of $[Z]$ on the set of all finite multisets of segments in irreducible cuspidal representations of general linear groups. This partial order satisfies that $L\left(\delta\left(\Delta_{1}^{\prime}\right), \ldots, \delta\left(\Delta_{k^{\prime}}^{\prime}\right)\right)$ is a subquotient of $\delta\left(\Delta_{1}\right) \times \cdots \times \delta\left(\Delta_{k}\right)$ if and only if we have for the multisets $\left(\Delta_{1}^{\prime}, \ldots, \Delta_{k^{\prime}}^{\prime}\right) \leq\left(\Delta_{1}, \ldots, \Delta_{k}\right)$ (see [T1] for example). If $\left(\Delta_{1}^{\prime}, \ldots, \Delta_{k^{\prime}}^{\prime}\right)$ is minimal or maximal among all multisets which satisfy $\left(\Delta_{1}^{\prime}, \ldots, \Delta_{k^{\prime}}^{\prime}\right) \leq\left(\Delta_{1}, \ldots, \Delta_{k}\right)$, then the multiplicity of $L\left(\delta\left(\Delta_{1}^{\prime}\right), \ldots, \delta\left(\Delta_{k^{\prime}}^{\prime}\right)\right)$ in $\delta\left(\Delta_{1}\right) \times \cdots \times \delta\left(\Delta_{k}\right)$ is one (Proposition 3.5 of [T1] implies the multiplicity one if $\left(\Delta_{1}^{\prime}, \ldots, \Delta_{k^{\prime}}^{\prime}\right)$ is minimal; if it is maximal, then this is a well known property of the Langlands' classification).

Let $J_{n}=\left(\delta_{i, n+1-j}\right)_{1 \leq i, j \leq n}$ be an $n \times n$ matrix, where $\delta_{i, n+1-j}$ denotes the Kronecker symbol. For a square matrix g, we shall denote by ${ }^{t} g$ the transposed matrix of $g$, and by ${ }^{\tau} g$ the transposed matrix with respect to the second diagonal. We fix one of the series of classical groups

$$
\begin{aligned}
S p(n, F) & =\left\{g \in G L(2 n, F) ;\left[\begin{array}{cc}
0 & -J_{n} \\
J_{n} & 0
\end{array}\right]{ }^{t} S\left[\begin{array}{cc}
0 & J_{n} \\
-J_{n} & 0
\end{array}\right]=S^{-1}\right\}, \\
S O(2 n+1, F) & =\left\{g \in G L(2 n+1, F) ;{ }^{\tau} S=S^{-1}\right\},
\end{aligned}
$$

and denote by $S_{n}$ the above group belonging to the series that we have fixed. For a partition $\alpha=\left(n_{1}, \ldots, n_{k}\right)$ of $m \leq n$, let

$$
\alpha^{\prime}=\left(n_{1}, n_{2}, \ldots, n_{k}, n^{\prime}-2 m, n_{k}, n_{k-1}, \ldots, n_{1}\right),
$$

where $n^{\prime}=2 n($ resp. $2 n+1)$ if $S_{n}=S p(n, F)$ (resp. $\left.S O(2 n+1, F)\right)$. Denote $P_{\alpha}=S_{n} \cap P_{\alpha^{\prime}}^{G L}, M_{\alpha}=S_{n} \cap M_{\alpha^{\prime}}^{G L}$. Using the isomorphism $\left(g_{1}, \ldots, g_{k}, h\right) \mapsto$ q-diag $\left(g_{1}, \ldots, g_{k}, h,{ }^{\tau} g_{k}^{-1}, \ldots,{ }^{\tau} g_{1}^{-1}\right)$, we shall identify $G L\left(n_{1}, F\right) \times \cdots \times G L\left(n_{k}, F\right) \times$ $S_{n-m}$ and $M_{\alpha}$. Therefore, each irreducible representation $\tau$ of $M_{\alpha}$ can be viewed as a tensor product $\pi_{1} \otimes \cdots \otimes \pi_{k} \otimes \sigma$, where all $\pi_{i}$ are irreducible representations of $G L\left(n_{i}, F\right)$ and $\sigma$ is an irreducible representation of $S_{n-m}$. Conversely, for irreducible representations $\pi_{i}$ of $G L\left(n_{i}, F\right)$ and an irreducible representation $\sigma$ of $S_{n-m}, \pi_{1} \otimes \cdots \otimes \pi_{k} \otimes \sigma$ can be considered as an irreducible representation of $M_{\alpha}$.

For admissible representations $\pi$ and $\sigma$ of $G L(k, F)$ and $S_{n-k}$ respectively, we consider $\pi \otimes \sigma$ as a representation of $M_{(k)}$, and define

$$
\pi \rtimes \sigma=\operatorname{Ind}_{P_{(k)}}^{S_{n}}(\pi \otimes \sigma) .
$$

If $\pi^{\prime}$ is additionally an admissible representation of a general linear group, then

$$
\pi^{\prime} \rtimes(\pi \rtimes \sigma) \cong\left(\pi^{\prime} \times \pi\right) \rtimes \sigma .
$$

Let $R_{n}(S)=\mathfrak{R}\left(S_{n}\right)$ and $R(S)=\bigoplus_{n \geq 0} R_{n}(S)$. Then $\rtimes$ lifts in a natural way to $\rtimes: R \times R(S) \rightarrow R(S)$, and $R(S)$ becomes graded $R$-module (note that $R \otimes R(S)$ 
is in a natural way an $R \otimes R$-module; we denote this action by $\rtimes$ again). In the $R$-module $R(S)$ the equality

$$
\pi \rtimes \sigma=\tilde{\pi} \rtimes \sigma \quad(\pi \in R, \sigma \in R(S))
$$

holds.

For an admissible representation $\pi$ of $S_{n}$ of finite length and a partition $\alpha$ of $m \leq n$, we denote by $s_{\alpha}(\pi)$ the normalized Jacquet module of $\pi$ with respect to $P_{\alpha}$. Let

$$
\mu^{*}(\pi)=\sum_{i=0}^{n} \text { s.s. }\left(s_{(i)}(\pi)\right) \text {. }
$$

We can (and we shall) consider $\mu^{*}(\pi) \in R \otimes R(S)$. Extend $\mu^{*}$ additively to $\mu^{*}$ : $R(S) \rightarrow R \otimes R(S)$. Denote

$$
M^{*}=(m \otimes 1) \circ\left(\sim \otimes m^{*}\right) \circ s \circ m^{*} .
$$

Then,

$$
\mu^{*}(\pi \rtimes \sigma)=M^{*}(\pi) \rtimes \mu^{*}(\sigma) .
$$

Let $\rho_{i}, i=1, \ldots, m$, be irreducible cuspidal representations of general linear groups, and let $\sigma$ be a similar representation of $S_{q}$. Suppose that $\rho_{1} \times \cdots \times \rho_{m}$ is a representation of $G L(a, F)$. If $\pi$ is a subquotient of $\rho_{1} \times \cdots \times \rho_{m} \rtimes \sigma$, then we shall denote $s_{(a)}(\pi)$ also by

$$
s_{G L}(\pi) .
$$

Let $\tau \otimes \sigma$ be a subquotient of $s_{G L}(\pi)$. If there exist irreducible cuspidal representations $\rho_{1}^{\prime}, \ldots, \rho_{m^{\prime}}^{\prime}$ of general linear groups such that $\tau \leq \rho_{1}^{\prime} \times \cdots \times \rho_{m^{\prime}}^{\prime}$, then we shall say that the multiset $\left(\rho_{1}^{\prime}, \ldots, \rho_{m^{\prime}}^{\prime}\right)$ is the $G L$-support of $\tau \otimes \sigma$ (in other words, the $G L$-support of $\tau \otimes \sigma$ is just the support of $\tau$ in the sense of [Z]).

We shall need the following fact proved in [T4].

1.1. Proposition. Let $\rho$ and $\sigma$ be irreducible unitarizable cuspidal representations of $G L(p, F)$ and $S_{q}$, respectively. Suppose that $\nu^{\alpha} \rho \rtimes \sigma$ reduces for some $\alpha>0$ and let $l$ be a nonnegative integer. Then the representation $\delta\left(\left[\nu^{\alpha} \rho, \nu^{\alpha+l} \rho\right]\right) \rtimes \sigma$ contains a unique irreducible subrepresentation, which we denote by $\delta\left(\left[\nu^{\alpha} \rho, \nu^{\alpha+l} \rho\right], \sigma\right)$. This subrepresentation is square integrable and we have $s_{G L}\left(\delta\left(\left[\nu^{\alpha} \rho, \nu^{\alpha+l} \rho\right], \sigma\right)\right)=$ $\delta\left(\left[\nu^{\alpha} \rho, \nu^{\alpha+l} \rho\right]\right) \otimes \sigma$. Moreover, $\mu^{*}\left(\delta\left(\left[\nu^{\alpha} \rho, \nu^{\alpha+l} \rho\right], \sigma\right)\right)=\sum_{k=-1}^{l} \delta\left(\left[\nu^{\alpha+k+1} \rho, \nu^{\alpha+l} \rho\right]\right)$ $\otimes \delta\left(\left[\nu^{\alpha} \rho, \nu^{\alpha+k} \rho\right], \sigma\right)$ (in this formula $\delta(\emptyset, \sigma)$ denotes $\left.\sigma\right)$.

Now, we shall recall of the Langlands' classification for the groups $S_{n}$. Let $\delta_{1}, \ldots, \delta_{k}$ be irreducible essentially square integrable representations of general linear groups which satisfy $e\left(\delta_{i}\right)>0, i=1, \ldots, k$, and let $\tau$ be an irreducible tempered representation of $S_{q}$. Choose a permutation $p$ of $\{1, \ldots, k\}$ such that $e\left(\delta_{p(1)}\right) \geq$ $\left(\delta_{p(2)}\right) \geq \cdots \geq e\left(\delta_{p(k)}\right)$. Then the representation $\delta_{p(1)} \times \delta_{p(2)} \times \cdots \times \delta_{p(k)} \rtimes \tau$ has a unique irreducible quotient, which will be denoted by $L\left(\delta_{1}, \ldots, \delta_{k}, \tau\right)$. Then $L\left(\delta_{1}, \ldots, \delta_{k}, \tau\right)^{\sim} \cong L\left(\delta_{1}, \ldots, \delta_{k}, \tilde{\tau}\right)$.

For positive integers $p$ and $k$ we shall denote the following partition of $p k$ by

$$
(p)^{k}=(\underbrace{p, p, \ldots, p}_{k \text { times }}) .
$$

Regarding sums and products, we shall have the following convention in this paper. Let $a, b \in(1 / 2) \mathbb{Z}$ such that $b-a \in \mathbb{Z}$. Suppose $a \leq b$. Then $\sum_{i=a}^{b} x_{i}$ (resp. 
$\left.\prod_{i=a}^{b} y_{i}\right)$ will denote $x_{a}+x_{a+1}+x_{a+2}+\cdots+x_{b}$ (resp. $\left.y_{a} y_{a+1} y_{a+2} \ldots y_{b}\right)$. If $a>b$, then we shall take $\sum_{i=a}^{b} x_{i}=0$.

\section{TEMPERED REPRESENTATIONS CORRESPONDING TO SEGMENTS}

In this section we shall fix an irreducible unitarizable cuspidal representation $\rho$ of $G L(p, F)$ and an irreducible cuspidal representation $\sigma$ of $S_{q}$, such that there exists $\alpha \geq 0$ in $(1 / 2) \mathbb{Z}$ so that $\nu^{\alpha} \rho \rtimes \sigma$ reduces (which implies $\rho \cong \tilde{\rho}$ ), and that $\nu^{\beta} \rho \rtimes \sigma$ is irreducible for all $\beta \in(\alpha+\mathbb{Z}) \backslash\{ \pm \alpha\}$. We shall also fix $n \in \mathbb{Z}, n \geq 0$ in this section. Denote

$$
\epsilon(\alpha)=\min \{\alpha-i ; i \in \mathbb{Z} \text { and } \alpha-i \geq 0\} .
$$

Clearly, $\epsilon(\alpha) \in\{0,1 / 2\}$ and $\epsilon(\alpha) \leq \alpha$.

2.1. Lemma. Let $\tau$ be an irreducible subquotient of $\delta\left(\left[\nu^{-\alpha-n} \rho, \nu^{\alpha+n} \rho\right]\right) \rtimes \sigma$. Then

$$
s_{G L}(\tau) \geq \sum_{i=\alpha+1}^{\alpha+n+1} \delta\left(\left[\nu^{i} \rho, \nu^{\alpha+n} \rho\right]\right) \times \delta\left(\left[\nu^{-i+1} \rho, \nu^{\alpha+n} \rho\right]\right) \otimes \sigma .
$$

If $\delta\left(\left[\nu^{-\alpha-n} \rho, \nu^{\alpha+n} \rho\right]\right) \rtimes \sigma$ reduces, then

$$
s_{G L}(\tau) \leq \sum_{i=-\alpha+1}^{\alpha+n+1} \delta\left(\left[\nu^{i} \rho, \nu^{\alpha+n} \rho\right]\right) \times \delta\left(\left[\nu^{-i+1} \rho, \nu^{\alpha+n} \rho\right]\right) \otimes \sigma .
$$

Proof. First note that the representation $\delta\left(\left[\nu^{-\alpha-n} \rho, \nu^{\alpha+n} \rho\right]\right) \rtimes \sigma$ is unitarizable. Clearly $s_{G L}(\tau) \leq s_{G L}\left(\delta\left(\left[\nu^{-\alpha-n} \rho, \nu^{\alpha+n} \rho\right]\right) \rtimes \sigma\right)$. We obtain from (1-1) and (1-4)

$$
\begin{aligned}
& \text { s.s. }\left(s_{G L}\left(\delta\left(\left[\nu^{-\alpha-n} \rho, \nu^{\alpha+n} \rho\right]\right) \rtimes \sigma\right)\right) \\
& \quad=\sum_{i=-\alpha-n}^{\alpha+n+1} \delta\left(\left[\nu^{i} \rho, \nu^{\alpha+n} \rho\right]\right) \times \delta\left(\left[\nu^{-i+1} \rho, \nu^{\alpha+n} \rho\right]\right) \otimes \sigma .
\end{aligned}
$$

Note that all the terms on the right-hand side of (2-3) are irreducible, and the terms corresponding to indexes $i$ and $-i+1$ are isomorphic. The multiplicity of $\delta\left(\left[\nu^{-\alpha-n} \rho, \nu^{\alpha+n} \rho\right]\right) \otimes \sigma$ in (2-3) is two. Frobenius reciprocity implies that if $\delta\left(\left[\nu^{-\alpha-n} \rho, \nu^{\alpha+n} \rho\right]\right) \rtimes \sigma$ reduces, then it reduces into two non-equivalent irreducible representations.

The unitarizability of $\delta\left(\left[\nu^{-\alpha-n} \rho, \nu^{\alpha+n} \rho\right]\right) \rtimes \sigma$ implies that $\tau$ is a subrepresentation of $\delta\left(\left[\nu^{-\alpha-n} \rho, \nu^{\alpha+n} \rho\right]\right) \rtimes \sigma$. Thus

$$
\begin{aligned}
& \tau \hookrightarrow \delta\left(\left[\nu^{-\alpha-n} \rho, \nu^{\alpha+n} \rho\right]\right) \rtimes \sigma \\
& \hookrightarrow \nu^{\alpha+n} \rho \times \nu^{\alpha+n-1} \rho \times \cdots \times \nu^{-\alpha-n+1} \rho \times \nu^{-\alpha-n} \rho \rtimes \sigma .
\end{aligned}
$$

Frobenius reciprocity implies $s_{G L}(\tau) \geq \delta\left(\left[\nu^{-\alpha-n} \rho, \nu^{\alpha+n} \rho\right]\right) \otimes \sigma$. If $n=0$, then this implies that (2-1) holds. Suppose $n \geq 1$. Now we shall prove that

$$
\begin{aligned}
\tau \hookrightarrow\left(\nu^{\alpha+n} \rho \times \nu^{\alpha+n-1} \rho \times \cdots\right. & \left.\times \nu^{\epsilon(\alpha)} \rho\right) \times\left(\nu^{\alpha+n} \rho \times \nu^{\alpha+n-1} \rho \times \cdots \times \nu^{i} \rho\right) \\
& \times\left(\nu^{\epsilon(\alpha)-1} \rho \times \nu^{\epsilon(\alpha)-2} \rho \times \cdots \times \nu^{-i+1} \rho\right) \rtimes \sigma
\end{aligned}
$$

for $i=\alpha+1, \alpha+2, \ldots, \alpha+n+1$.

If $i=\alpha+n+1$, then (2-5) holds because (2-4) holds. Suppose $\alpha+1<i \leq \alpha+n+1$, and suppose that (2-5) holds for this $i$. First denote by

$$
\pi=\left(\nu^{\alpha+n} \rho \times \nu^{\alpha+n-1} \rho \times \cdots \times \nu^{\epsilon(\alpha)} \rho\right) \times\left(\nu^{\alpha+n} \rho \times \nu^{\alpha+n-1} \rho \times \cdots \times \nu^{i} \rho\right) .
$$


Note that $-i<-\alpha-1$, and further $-i+1<-\alpha$. Therefore, $\nu^{-i+1} \rho \rtimes \sigma$ is irreducible. This implies $\nu^{-i+1} \rho \rtimes \sigma \cong \nu^{i-1} \rho \rtimes \sigma$. Using this and the fact that $\epsilon(\alpha) \leq \alpha<i-1$ (which implies $\nu^{j} \rho \times \nu^{i-1} \rho \cong \nu^{i-1} \rho \times \nu^{j} \rho$ for $-i+2 \leq j \leq \epsilon(\alpha)-1$, because of the irreducibility), we get from (2-5) (which we have supposed to hold for $i$ )

$$
\begin{aligned}
& \tau \hookrightarrow \pi \times\left(\nu^{\epsilon(\alpha)-1} \rho \times \nu^{\epsilon(\alpha)-2} \rho \times \cdots \times \nu^{-i+1} \rho\right) \rtimes \sigma \\
& \cong \pi \times\left(\nu^{\epsilon(\alpha)-1} \rho \times \nu^{\epsilon(\alpha)-2} \rho \times \cdots \times \nu^{-i+2} \rho\right) \rtimes\left(\nu^{-i+1} \rho \rtimes \sigma\right) \\
& \cong \pi \times\left(\nu^{\epsilon(\alpha)-1} \rho \times \nu^{\epsilon(\alpha)-2} \rho \times \cdots \times \nu^{-i+2} \rho\right) \rtimes\left(\nu^{i-1} \rho \rtimes \sigma\right) \\
& \cong \pi \times\left(\nu^{\epsilon(\alpha)-1} \rho \times \nu^{\epsilon(\alpha)-2} \rho \times \cdots \times \nu^{-i+3} \rho\right) \times\left(\nu^{-i+2} \rho \times \nu^{i-1} \rho\right) \rtimes \sigma \\
& \cong \pi \times\left(\nu^{\epsilon(\alpha)-1} \rho \times \nu^{\epsilon(\alpha)-2} \rho \times \cdots \times \nu^{-i+3} \rho\right) \times\left(\nu^{i-1} \rho \times \nu^{-i+2} \rho\right) \rtimes \sigma \\
& \cong \pi \times\left(\nu^{\epsilon(\alpha)-1} \rho \times \nu^{\epsilon(\alpha)-2} \rho \times \cdots \times \nu^{-i+4} \rho\right) \times\left(\nu^{-i+3} \rho \times \nu^{i-1} \rho\right) \times \nu^{-i+2} \rho \rtimes \sigma \\
& \cong \pi \times\left(\nu^{\epsilon(\alpha)-1} \rho \times \nu^{\epsilon(\alpha)-2} \rho \times \cdots \times \nu^{-i+4} \rho\right) \times\left(\nu^{i-1} \rho \times \nu^{-i+3} \rho\right) \times \nu^{-i+2} \rho \rtimes \sigma
\end{aligned}
$$

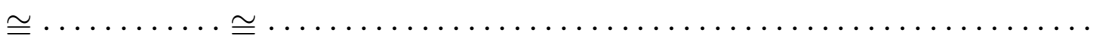

$$
\begin{aligned}
& \cong \pi \times \nu^{i-1} \rho \times\left(\nu^{\epsilon(\alpha)-1} \rho \times \nu^{\epsilon(\alpha)-2} \rho \times \cdots \times \nu^{-i+3} \rho \times \nu^{-i+2} \rho\right) \rtimes \sigma \\
& \cong\left(\nu^{\alpha+n} \rho \times \nu^{\alpha+n-1} \rho \times \cdots \times \nu^{\epsilon(\alpha)} \rho\right) \times\left(\nu^{\alpha+n} \rho \times \nu^{\alpha+n-1} \rho \times \cdots \times \nu^{i} \rho \times \nu^{i-1} \rho\right) \\
& \times\left(\nu^{\epsilon(\alpha)-1} \rho \times \nu^{\epsilon(\alpha)-2} \rho \times \cdots \times \nu^{-i+3} \rho \times \nu^{-i+2} \rho\right) \rtimes \sigma .
\end{aligned}
$$

Note that (2-6) shows that (2-5) holds for $i-1$. Therefore, we have proved by induction that (2-5) holds.

Frobenius reciprocity implies from (2-5) that

$$
\begin{array}{r}
\left(\nu^{\alpha+n} \rho \otimes \nu^{\alpha+n-1} \rho \otimes \cdots \otimes \nu^{\epsilon(\alpha)} \rho\right) \otimes\left(\nu^{\alpha+n} \rho \otimes \nu^{\alpha+n-1} \rho \otimes \cdots \otimes \nu^{i} \rho\right) \\
\otimes\left(\nu^{\epsilon(\alpha)-1} \rho \otimes \nu^{\epsilon(\alpha)-2} \rho \otimes \cdots \otimes \nu^{-i+1} \rho\right) \otimes \sigma
\end{array}
$$

is a subquotient of $s_{(p)^{2(\alpha+n)+1}}(\tau)$, for $i=\alpha+1, \alpha+2, \ldots, \alpha+n+1$ (for the definition of $(p)^{2(\alpha+n)+1}$ see (1-5)). Considering $G L$-supports of the representations on the right-hand side of (2-3), the transitivity of Jacquet modules and (2-3) imply that we must have

$$
s_{G L}(\tau) \geq \delta\left(\left[\nu^{-i+1} \rho, \nu^{\alpha+n} \rho\right]\right) \times \delta\left(\left[\nu^{i} \rho, \nu^{\alpha+n} \rho\right]\right) \otimes \sigma
$$

for $\alpha+1 \leq i \leq \alpha+n+1$ (since the representation on the right-hand side is the only irreducible representation appearing in the sum (2-3) which can have (2-7) as a subquotient of a Jacquet module). Since the (irreducible) representations on the right-hand side of (2-8), for $i=\alpha+1, \ldots, \alpha+n+1$, are not equivalent (they have different $G L$-supports), (2-8) implies (2-1).

Suppose that $\delta\left(\left[\nu^{-\alpha-n} \rho, \nu^{\alpha+n} \rho\right]\right) \rtimes \sigma$ reduces. Then this representation is a direct sum of $\tau$ and an irreducible tempered representation $\tau^{\prime}$. Since $s_{G L}\left(\tau^{\prime}\right)$ satisfy the estimate (2-1), we get (2-2) from (2-3) and (2-1).

2.2. Lemma. The multiplicity of

$$
\delta\left(\left[\nu^{\epsilon(\alpha)} \rho, \nu^{\alpha+n} \rho\right]\right) \times \delta\left(\left[\nu^{1-\epsilon(\alpha)} \rho, \nu^{\alpha+n} \rho\right]\right) \otimes \sigma
$$

in $s_{G L}\left(\delta\left(\left[\nu^{-\alpha-n} \rho, \nu^{\alpha+n} \rho\right]\right) \rtimes \sigma\right)$ and $s_{G L}\left(\left(\prod_{i=-\alpha-n}^{\alpha+n} \nu^{i} \rho\right) \rtimes \sigma\right)$ is $2(1-\epsilon(\alpha))$ (i.e., it is two if $\alpha \in \mathbb{Z}$ and one otherwise). 
Proof. Write

$$
\begin{aligned}
\text { s.s. }\left(s_{G L}\left(\left(\prod_{i=-\alpha-n}^{\alpha+n} \nu^{i} \rho\right) \rtimes \sigma\right)\right)= & \left(\prod_{i=-\alpha-n}^{\alpha+n}\left(\nu^{i} \rho+\nu^{-i} \rho\right)\right) \otimes \sigma \\
= & \sum_{\epsilon_{-\alpha-n}, \epsilon_{-\alpha-n+1}, \ldots, \epsilon_{\alpha+n} \in\{ \pm 1\}}\left(\prod_{i=-\alpha-n}^{\alpha+n} \nu^{\epsilon_{i} i} \rho\right) \otimes \sigma .
\end{aligned}
$$

Suppose $\alpha \in \mathbb{Z}$. From (2-3) we get $\delta\left(\left[\rho, \nu^{\alpha+n} \rho\right]\right) \times \delta\left(\left[\nu \rho, \nu^{\alpha+n} \rho\right]\right) \otimes \sigma$ as a subquotient of $s_{G L}\left(\delta\left(\left[\nu^{-\alpha-n} \rho, \nu^{\alpha+n} \rho\right]\right) \rtimes \sigma\right.$ for terms corresponding to $i=0$ and 1 , and only then. Thus, the multiplicity is two. To get $\delta\left(\left[\rho, \nu^{\alpha+n} \rho\right]\right) \times \delta\left(\left[\nu \rho, \nu^{\alpha+n} \rho\right]\right) \otimes \sigma$ as a subquotient of (2-10), we must have $\epsilon_{i} i \geq 0$ for all $i$ in the sum (2-10). This implies $\epsilon_{i}=\operatorname{sgn}(i)$ for $i \neq 0$. Therefore, there are only two terms in the sum (2-10) where $\delta\left(\left[\rho, \nu^{\alpha+n} \rho\right]\right) \times \delta\left(\left[\nu \rho, \nu^{\alpha+n} \rho\right]\right) \otimes \sigma$ can be a subquotient (they correspond to $\epsilon_{i}=\operatorname{sgn}(i)$ for $i \neq 0$ and $\left.\epsilon_{0}= \pm 1\right)$. In each of these two terms the multiplicity is one. This ends the proof of the lemma in the case $\alpha \in \mathbb{Z}$. The proof for $\alpha \in(1 / 2+\mathbb{Z})$ is analogous (here all $\epsilon_{i} i$ must be positive, which implies $\epsilon_{i}=\operatorname{sgn}(i)$ for all $i)$.

2.3. Theorem. Let $\rho$ be an irreducible unitarizable cuspidal representation of $G L(p, F)$, let $\sigma$ be an irreducible cuspidal representation of $S_{q}$ and let $n \in \mathbb{Z}, n \geq 0$. Suppose that $\rho \rtimes \sigma$ reduces and that $\nu^{i} \rho \rtimes \sigma$ is irreducible for all $i \in \mathbb{Z} \backslash\{0\}$. Then $\rho \rtimes \sigma=\tau_{1} \oplus \tau_{2}$, where $\tau_{1}$ and $\tau_{2}$ are inequivalent irreducible representations. The representation $\delta\left(\left[\nu^{-n} \rho, \nu^{n} \rho\right]\right) \rtimes \sigma$ splits into a sum of two inequivalent irreducible representations. Each of them can be characterized as a unique common irreducible subquotient of $\delta\left(\left[\nu^{-n} \rho, \nu^{n} \rho\right]\right) \rtimes \sigma$ and $\delta\left(\left[\nu \rho, \nu^{n} \rho\right]\right)^{2} \rtimes \tau_{i}$. This subquotient will be denoted by

$$
\delta\left(\left[\nu^{-n} \rho, \nu^{n} \rho\right]_{\tau_{i}}, \sigma\right)
$$

We have

$$
\begin{gathered}
s_{G L}\left(\delta\left(\left[\nu^{-n} \rho, \nu^{n} \rho\right]_{\tau_{i}}, \sigma\right)\right)=\sum_{j=1}^{n+1} \delta\left(\left[\nu^{j} \rho, \nu^{n} \rho\right]\right) \times \delta\left(\left[\nu^{-j+1} \rho, \nu^{n} \rho\right]\right) \otimes \sigma, \\
\delta\left(\left[\nu^{-n} \rho, \nu^{n} \rho\right]_{\tau_{i}}, \sigma\right)^{\sim} \cong \delta\left(\left[\nu^{-n} \rho, \nu^{n} \rho\right] \tilde{\tau}_{i}, \tilde{\sigma}\right) .
\end{gathered}
$$

If $\pi$ is a subquotient of $\left(\prod_{j=-\alpha-n}^{\alpha+n} \nu^{j} \rho\right) \rtimes \sigma$ which has $\delta\left(\left[\rho, \nu^{n} \rho\right]\right) \times \delta\left(\left[\nu \rho, \nu^{n} \rho\right]\right) \otimes \sigma$ as a subquotient of $s_{G L}(\pi)$, then $\pi \cong \delta\left(\left[\nu^{-n} \rho, \nu^{n} \rho\right]_{\tau_{i}}, \sigma\right)$ for some $i \in\{1,2\}$.

Proof. The reducibility of $\delta\left(\left[\nu^{-n} \rho, \nu^{n} \rho\right]\right) \rtimes \sigma$ follows from Proposition 4.8 of [T5] (we shall see this below again). The proof of Lemma 2.1 implies that $\delta\left(\left[\nu^{-n} \rho, \nu^{n} \rho\right]\right) \rtimes \sigma$ (and also $\rho \rtimes \sigma$ ) is a direct sum of two inequivalent irreducible representations. Further, the multiplicity of $\delta\left(\left[\rho, \nu^{n} \rho\right]\right) \times \delta\left(\left[\nu \rho, \nu^{n} \rho\right]\right) \otimes \sigma$ in

$$
\text { S.s. }\left(s_{G L}\left(\delta\left(\left[\nu \rho, \nu^{n} \rho\right]\right)^{2} \rtimes \tau_{i}\right)\right)=\left(\sum_{j=1}^{n+1} \delta\left(\left[\nu^{j} \rho, \nu^{n} \rho\right]\right) \times \delta\left(\left[\nu^{-j+1} \rho, \nu^{-1} \rho\right]\right)\right)^{2} \times \rho \otimes \sigma
$$

is one. Lemma 2.2 tells us that the multiplicity of $\delta\left(\left[\rho, \nu^{n} \rho\right]\right) \times \delta\left(\left[\nu \rho, \nu^{n} \rho\right]\right) \otimes \sigma$ in representations $s_{G L}\left(\delta\left(\left[\nu^{-n} \rho, \nu^{n} \rho\right]\right) \rtimes \sigma\right)$ and $s_{G L}\left(\left(\prod_{j=-n}^{n} \nu^{j} \rho\right) \rtimes \sigma\right)$ is two. This implies the reducibility of $\delta\left(\left[\nu^{-n} \rho, \nu^{n} \rho\right]\right) \rtimes \sigma$, also the uniqueness of the common irreducible subquotient of $\delta\left(\left[\nu^{-n} \rho, \nu^{n} \rho\right]\right) \rtimes \sigma$ and $\delta\left(\left[\nu \rho, \nu^{n} \rho\right]\right)^{2} \rtimes \tau_{i}$ (therefore, 
$\delta\left(\left[\nu^{-n} \rho, \nu^{n} \rho\right]_{\tau_{i}}, \sigma\right)$ is well defined in the theorem $)$. Passing to the contragredients, we get that $\rho \rtimes \tilde{\sigma} \cong \tilde{\rho} \rtimes \tilde{\sigma}=\tilde{\tau}_{1} \oplus \tilde{\tau}_{2}$, and that $\delta\left(\left[\nu^{-n} \rho, \nu^{n} \rho\right]_{\tau_{i}}, \sigma\right)^{\sim}$ is a common irreducible subquotient of $\left(\delta\left(\left[\nu^{-n} \rho, \nu^{n} \rho\right]\right) \rtimes \sigma\right)^{\sim} \cong \delta\left(\left[\nu^{-n} \rho, \nu^{n} \rho\right]\right) \rtimes \tilde{\sigma}$ and $\left(\delta\left(\left[\nu \rho, \nu^{n} \rho\right]\right)^{2} \rtimes\right.$ $\left.\tau_{i}\right)^{\sim} \cong \delta\left(\left[\nu^{-n} \rho, \nu^{-1} \rho\right]\right)^{2} \rtimes \tilde{\tau}_{i}$. From (1-3) we get s.s. $\left(\delta\left(\left[\nu^{-n} \rho, \nu^{-1} \rho\right]\right)^{2} \rtimes \tilde{\tau}_{i}\right)=$ s.s. $\left(\delta\left(\left[\nu \rho, \nu^{n} \rho\right]\right)^{2} \rtimes \tilde{\tau}_{i}\right)$. Therefore, $\delta\left(\left[\nu^{-n} \rho, \nu^{n} \rho\right]_{\tau_{i}}, \sigma\right)^{\sim}$ is a common irreducible subquotient of $\delta\left(\left[\nu^{-n} \rho, \nu^{n} \rho\right]\right) \rtimes \tilde{\sigma}$ and $\delta\left(\left[\nu \rho, \nu^{n} \rho\right]\right)^{2} \rtimes \tilde{\tau}_{i}$. The definition of $\delta\left(\left[\nu^{-n} \rho\right.\right.$, $\left.\left.\nu^{n} \rho\right]_{\tilde{\tau}_{i}}, \sigma\right)$ now implies $\delta\left(\left[\nu^{-n} \rho, \nu^{n} \rho\right]_{\tau_{i}}, \sigma\right)^{\sim} \cong \delta\left(\left[\nu^{-n} \rho, \nu^{n} \rho\right]_{\tilde{\tau}_{i}}, \sigma\right)$. This proves (212). Inequalities (2-1) and (2-2) imply (2-11).

Lemma 2.2 and exactness of the Jacquet functors imply that if $\pi$ is a subquotient of $\left(\prod_{j=-\alpha-n}^{\alpha+n} \nu^{j} \rho\right) \rtimes \sigma$ having $\delta\left(\left[\rho, \nu^{n} \rho\right]\right) \times \delta\left(\left[\nu \rho, \nu^{n} \rho\right]\right) \otimes \sigma$ for a subquotient of $s_{G L}(\pi)$, then $\pi \leq \delta\left(\left[\nu^{-n} \rho, \nu^{n} \rho\right]\right) \rtimes \sigma$. The first part of the proof now implies $\pi \cong \delta\left(\left[\nu^{-n} \rho, \nu^{n} \rho\right]_{\tau_{i}}, \sigma\right)$ for some $i \in\{1,2\}$.

In the rest of this section we shall assume $\alpha>0$.

2.4. Lemma. Let $\alpha>0$.

(i) Suppose $-\alpha+1 \leq i \leq \alpha$ (which is equivalent to $-\alpha+1 \leq i$ and $-\alpha+1 \leq$ $-i+1)$. Then the multiplicity of

$$
\delta\left(\left[\nu^{i} \rho, \nu^{\alpha+n} \rho\right]\right) \times \delta\left(\left[\nu^{-i+1} \rho, \nu^{\alpha+n} \rho\right]\right) \otimes \sigma
$$

in all the following representations

$$
\begin{gathered}
s_{G L}\left(\delta\left(\left[\nu^{-\alpha-n} \rho, \nu^{\alpha+n} \rho\right]\right) \rtimes \sigma\right), \\
s_{G L}\left(\delta\left(\left[\nu^{-\alpha+1} \rho, \nu^{\alpha+n} \rho\right]\right) \rtimes \delta\left(\left[\nu^{\alpha} \rho, \nu^{\alpha+n} \rho\right], \sigma\right)\right), \\
\left.s_{G L}\left(\delta\left(\left[\nu^{-\alpha+1} \rho, \nu^{\alpha+n} \rho\right]\right) \rtimes \delta\left(\left[\nu^{\alpha} \rho, \nu^{\alpha+n} \rho\right]\right) \rtimes \sigma\right)\right)
\end{gathered}
$$

is two, except if $\alpha \in 1 / 2+\mathbb{Z}$ and $i=1 / 2$, when the multiplicity is one.

(ii) The multiplicity of $\delta\left(\left[\nu^{-\alpha-n} \rho, \nu^{\alpha+n} \rho\right]\right) \otimes \sigma$ in $(2-14)$ is 2.

(iii) The multiplicity of $\delta\left(\left[\nu^{-\alpha-n} \rho, \nu^{\alpha+n} \rho\right]\right) \otimes \sigma$ in $(2-15)$ is 1 .

Proof. From (2-3) we see that the multiplicities claimed in (i) for

$$
s_{G L}\left(\delta\left(\left[\nu^{-\alpha-n} \rho, \nu^{\alpha+n} \rho\right]\right) \rtimes \sigma\right)
$$

holds (one gets multiplicity two in the case $i \neq 1 / 2$ from the fact that the representations in the sum (2-3) corresponding to $i$ and $-i+1$ are isomorphic).

Further, write

$$
\begin{aligned}
& \text { s.s. }\left(s_{G L}\left(\delta\left(\left[\nu^{-\alpha+1} \rho, \nu^{\alpha+n} \rho\right]\right) \rtimes \delta\left(\left[\nu^{\alpha} \rho, \nu^{\alpha+n} \rho\right], \sigma\right)\right)\right) \\
& =\left(\sum_{j=-\alpha+1}^{\alpha+n+1} \delta\left(\left[\nu^{-j+1} \rho, \nu^{\alpha-1} \rho\right]\right) \times \delta\left(\left[\nu^{j} \rho, \nu^{\alpha+n} \rho\right]\right)\right) \times \delta\left(\left[\nu^{\alpha} \rho, \nu^{\alpha+n} \rho\right]\right) \otimes \sigma .
\end{aligned}
$$

We are looking at the multiplicities of $\delta\left(\left[\nu^{i} \rho, \nu^{\alpha+n} \rho\right]\right) \times \delta\left(\left[\nu^{-i+1} \rho, \nu^{\alpha+n} \rho\right]\right) \otimes \sigma$ for $-\alpha+1 \leq i \leq \alpha$ (then $-\alpha+1 \leq i$ and $-\alpha+1 \leq-i+1)$. Considering supports, we see that we need to consider only the terms in the above sum corresponding to $-\alpha+1 \leq j \leq \alpha$, and we see that we can get $\delta\left(\left[\nu^{i} \rho, \nu^{\alpha+n} \rho\right]\right) \times \delta\left(\left[\nu^{-i+1} \rho, \nu^{\alpha+n} \rho\right]\right) \otimes \sigma$ as a subquotient of the $j$-th term in the sum (2-17) if and only $j=i$ or $j=-i+1$ (note that $-\alpha+1 \leq-i+1 \leq \alpha$ ), and in these cases we have the multiplicity one of $\delta\left(\left[\nu^{i} \rho, \nu^{\alpha+n} \rho\right]\right) \times \delta\left(\left[\nu^{-i+1} \rho, \nu^{\alpha+n} \rho\right]\right) \otimes \sigma$ in that term. This ends the proof that the multiplicity claimed in (i) holds for (2-15). 
Write now

$$
\begin{aligned}
\text { s.s. } \left.\left(s_{G L}\left(\delta\left(\left[\nu^{-\alpha+1} \rho, \nu^{\alpha+n} \rho\right]\right) \times \delta\left(\left[\nu^{\alpha} \rho, \nu^{\alpha+n} \rho\right]\right) \rtimes \sigma\right)\right)\right) \\
=\left(\sum_{j=-\alpha+1}^{\alpha+n+1} \delta\left(\left[\nu^{-j+1} \rho, \nu^{\alpha-1} \rho\right]\right) \times \delta\left(\left[\nu^{j} \rho, \nu^{\alpha+n} \rho\right]\right)\right) \\
\quad \times\left(\sum_{k=\alpha}^{\alpha+n+1} \delta\left(\left[\nu^{-k+1} \rho, \nu^{-\alpha} \rho\right]\right) \times \delta\left(\left[\nu^{k} \rho, \nu^{\alpha+n} \rho\right]\right)\right) \otimes \sigma .
\end{aligned}
$$

Since $\nu^{-\alpha} \rho$ is not in the $G L$-support of $\delta\left(\left[\nu^{i} \rho, \nu^{\alpha+n} \rho\right]\right) \times \delta\left(\left[\nu^{-i+1} \rho, \nu^{\alpha+n} \rho\right]\right) \otimes \sigma$, to have $\delta\left(\left[\nu^{i} \rho, \nu^{\alpha+n} \rho\right]\right) \times \delta\left(\left[\nu^{-i+1} \rho, \nu^{\alpha+n} \rho\right]\right) \otimes \sigma$ for a subquotient of a term in the sum (2-18), we need to take $k=\alpha$. Therefore, the multiplicity is the same as in $(2-17)$, and this case we have already checked. Now the proof of (i) is complete.

Further, (ii) is evident from (2-3). Also, (iii) follows directly from (2-17). In both cases we need to use that $\nu^{-\alpha-n} \rho$ is in the $G L$-support of $\delta\left(\left[\nu^{-\alpha-n} \rho, \nu^{\alpha+n} \rho\right]\right) \otimes$ $\sigma$.

2.5. Theorem. Let $\rho$ be an irreducible unitarizable cuspidal representation of $G L(p, F)$, let $\sigma$ be an irreducible cuspidal representation of $S_{q}$, let $\alpha \in(1 / 2) \mathbb{Z}, \alpha>$ 0 and $n \in \mathbb{Z}, n \geq 0$. Assume that $\nu^{\alpha} \rho \rtimes \sigma$ reduces and that $\nu^{\beta} \rho \rtimes \sigma$ is irreducible for $\beta \in(\alpha+\mathbb{Z}) \backslash\{ \pm \alpha\}$.

(i) The representation $\delta\left(\left[\nu^{-\alpha-n} \rho, \nu^{\alpha+n} \rho\right]\right) \rtimes \sigma$ splits into a direct sum of two inequivalent irreducible representations.

(ii) The representations

$$
\delta\left(\left[\nu^{-\alpha-n} \rho, \nu^{\alpha+n} \rho\right]\right) \rtimes \sigma \quad \text { and } \quad \delta\left(\left[\nu^{-\alpha+1} \rho, \nu^{\alpha+n} \rho\right]\right) \rtimes \delta\left(\left[\nu^{\alpha} \rho, \nu^{\alpha+n} \rho\right], \sigma\right)
$$

have a unique irreducible subquotient in common, which we denote by

$$
\delta\left(\left[\nu^{-\alpha-n} \rho, \nu^{\alpha+n} \rho\right], \sigma\right) \quad \text { or } \quad \delta\left(\left[\nu^{-\alpha-n} \rho, \nu^{\alpha+n} \rho\right]_{+}, \sigma\right) .
$$

The other irreducible summand in $\delta\left(\left[\nu^{-\alpha-n} \rho, \nu^{\alpha+n} \rho\right]\right) \rtimes \sigma$ will be denoted by

$$
\delta\left(\left[\nu^{-\alpha-n} \rho, \nu^{\alpha+n} \rho\right]_{-}, \sigma\right) .
$$

(iii) We have

$$
\begin{aligned}
s_{G L}( & \left.\delta\left(\left[\nu^{-\alpha-n} \rho, \nu^{\alpha+n} \rho\right], \sigma\right)\right) \\
& =\sum_{i=-\alpha-n}^{\alpha} \delta\left(\left[\nu^{i} \rho, \nu^{\alpha+n} \rho\right]\right) \times \delta\left(\left[\nu^{-i+1} \rho, \nu^{\alpha+n} \rho\right]\right) \otimes \sigma, \\
s_{G L}( & \left.\delta\left(\left[\nu^{-\alpha-n} \rho, \nu^{\alpha+n} \rho\right]_{-}, \sigma\right)\right) \\
& =\sum_{i=-\alpha-n}^{-\alpha} \delta\left(\left[\nu^{i} \rho, \nu^{\alpha+n} \rho\right]\right) \times \delta\left(\left[\nu^{-i+1} \rho, \nu^{\alpha+n} \rho\right]\right) \otimes \sigma .
\end{aligned}
$$

(iv) Suppose that $\pi$ is a subquotient of $\left(\prod_{i=-\alpha-n}^{\alpha+n} \nu^{i} \rho\right) \rtimes \sigma$ such that

$$
\delta\left(\left[\nu^{\epsilon(\alpha)} \rho, \nu^{\alpha+n} \rho\right]\right) \times \delta\left(\left[\nu^{1-\epsilon(\alpha)} \rho, \nu^{\alpha+n} \rho\right]\right) \otimes \sigma
$$

is a subquotient of $s_{G L}(\pi)$. Then $\pi$ has multiplicity one in $\left(\prod_{i=-\alpha-n}^{\alpha+n} \nu^{i} \rho\right) \rtimes \sigma$ and $\pi \cong \delta\left(\left[\nu^{-\alpha-n} \rho, \nu^{\alpha+n} \rho\right], \sigma\right)$.

(v) $\delta\left(\left[\nu^{-\alpha-n} \rho, \nu^{\alpha+n} \rho\right]_{ \pm}, \sigma\right)^{\sim} \cong \delta\left(\left[\nu^{-\alpha-n} \rho, \nu^{\alpha+n} \rho\right]_{ \pm}, \tilde{\sigma}\right)$. 
Proof. From (2-3) we see that $\delta\left(\left[\nu^{-\alpha-n} \rho, \nu^{\alpha+n} \rho\right]\right) \rtimes \sigma$ is a multiplicity one representation of the length $\leq 2$ (see the proof of Lemma 2.1). Note that

$$
\begin{aligned}
& \delta\left(\left[\nu^{-\alpha-n} \rho, \nu^{\alpha+n} \rho\right]\right) \rtimes \sigma \leq \delta\left(\left[\nu^{-\alpha+1} \rho, \nu^{\alpha+n} \rho\right]\right) \times \delta\left(\left[\nu^{\alpha} \rho, \nu^{\alpha+n} \rho\right]\right) \rtimes \sigma, \\
& \delta\left(\left[\nu^{-\alpha+1} \rho, \nu^{\alpha+n} \rho\right]\right) \rtimes \delta\left(\left[\nu^{\alpha} \rho, \nu^{\alpha+n} \rho\right], \sigma\right) \\
& \quad \leq \delta\left(\left[\nu^{-\alpha+1} \rho, \nu^{\alpha+n} \rho\right]\right) \times \delta\left(\left[\nu^{\alpha} \rho, \nu^{\alpha+n} \rho\right]\right) \rtimes \sigma .
\end{aligned}
$$

Since $-\alpha+1 \leq \alpha \leq \alpha$, (i) of Lemma 2.4 and inequalities (2-21) (together with the exactness of Jacquet functors $)$, imply that representations $\delta\left(\left[\nu^{-\alpha-n} \rho, \nu^{\alpha+n} \rho\right]\right)$ $\rtimes \sigma$ and $\delta\left(\left[\nu^{-\alpha+1} \rho, \nu^{\alpha+n} \rho\right]\right) \rtimes \delta\left(\left[\nu^{\alpha} \rho, \nu^{\alpha+n} \rho\right], \sigma\right)$ have at least one irreducible subquotient in common. Further, (ii) and (iii) of Lemma 2.4 imply that $\delta\left(\left[\nu^{-\alpha-n} \rho, \nu^{\alpha+n} \rho\right]\right)$ $\rtimes \sigma$ is reducible and that it has a unique irreducible subquotient in common with $\delta\left(\left[\nu^{-\alpha+1} \rho, \nu^{\alpha+n} \rho\right]\right) \rtimes \delta\left(\left[\nu^{\alpha} \rho, \nu^{\alpha+n} \rho\right], \sigma\right)$. Denote this common irreducible subquotient by $\pi$. Let $\pi_{-}$be the other irreducible summand.

Suppose $\alpha \in \mathbb{Z}$. Lemma 2.4 (i) implies that the multiplicity of $\delta\left(\left[\nu^{i} \rho, \nu^{\alpha+n} \rho\right]\right) \times$ $\delta\left(\left[\nu^{-i+1} \rho, \nu^{\alpha+n} \rho\right]\right) \otimes \sigma$, with $-\alpha+1 \leq i \leq \alpha$, in

$$
\begin{gathered}
s_{G L}\left(\delta\left(\left[\nu^{-\alpha-n} \rho, \nu^{\alpha+n} \rho\right]\right) \rtimes \sigma\right), s_{G L}\left(\delta\left(\left[\nu^{-\alpha+1} \rho, \nu^{\alpha+n} \rho\right]\right) \rtimes \delta\left(\left[\nu^{\alpha} \rho, \nu^{\alpha+n} \rho\right], \sigma\right)\right) \\
\text { and } s_{G L}\left(\delta\left(\left[\nu^{-\alpha+1} \rho, \nu^{\alpha+n} \rho\right]\right) \times \delta\left(\left[\nu^{\alpha} \rho, \nu^{\alpha+n} \rho\right]\right) \rtimes \sigma\right)
\end{gathered}
$$

is two. Thus, the exactness of Jacquet functors, (2-21) and the characterization of $\pi$, imply

$$
s_{G L}(\pi) \geq \sum_{i=-\alpha+1}^{\alpha} \delta\left(\left[\nu^{i} \rho, \nu^{\alpha+n} \rho\right]\right) \times \delta\left(\left[\nu^{-i+1} \rho, \nu^{\alpha+n} \rho\right]\right) \otimes \sigma,
$$

since terms in the above sums corresponding to $i$ and $-i+1$ are equivalent, and these are the only equivalences among them.

Now let $\alpha \in(1 / 2)+\mathbb{Z}$. Lemma 2.4 (i) claims that the multiplicity of $\delta\left(\left[\nu^{i} \rho\right.\right.$, $\left.\left.\nu^{\alpha+n} \rho\right]\right) \times \delta\left(\left[\nu^{-i+1} \rho, \nu^{\alpha+n} \rho\right]\right) \otimes \sigma$, with $-\alpha+1 \leq i \leq \alpha$, in the Jacquet modules $(2-$ $22)$ is two, except if $i=1 / 2$, when the multiplicity is one. Again from this, (2-21), the exactness of Jacquet functors and the characterization of $\pi$ we get that (2-23) holds in this case (since the multiplicity of $\delta\left(\left[\nu^{i} \rho, \nu^{\alpha+n} \rho\right]\right) \times \delta\left(\left[\nu^{-i+1} \rho, \nu^{\alpha+n} \rho\right]\right) \otimes \sigma$, for $-\alpha+1 \leq i \leq \alpha$, in the right-hand side of inequality (2-23) is two, except for $i=1 / 2$, when we have the multiplicity one, and there are no other irreducible subquotients of the right-hand side of (2-23)).

We know that $\pi$ and $\pi_{-}$satisfy inequality (2-1). Since there is no common irreducible subquotient of right-hand sides of (2-1) and (2-23), (2-1) and (2-23) imply

$$
s_{G L}(\pi) \geq \sum_{i=-\alpha+1}^{\alpha+n+1} \delta\left(\left[\nu^{i} \rho, \nu^{\alpha+n} \rho\right]\right) \times \delta\left(\left[\nu^{-i+1} \rho, \nu^{\alpha+n} \rho\right]\right) \otimes \sigma,
$$

Now (2-2) implies that in (2-24) we have equality. Introducing a new index $j=$ $-i+1$ in the sum on the right-hand side of (2-24), we get (2-19). Since $\pi \oplus \pi_{-}=$ $\delta\left(\left[\nu^{-\alpha-n} \rho, \nu^{\alpha+n} \rho\right]\right) \rtimes \sigma,(2-3)$ and (2-19) imply (2-20).

Let $\alpha \in \mathbb{Z}$. Then (2-19) implies that the multiplicity of

$$
\delta\left(\left[\rho, \nu^{\alpha+n} \rho\right]\right) \times \delta\left(\left[\nu \rho, \nu^{\alpha+n} \rho\right]\right) \otimes \sigma
$$

in $s_{G L}(\pi)$ is $\geq 2$ (since $\left.-\alpha-n \leq 0,1 \leq \alpha\right)$. Now Lemma 2.2 and the exactness of Jacquet functors imply (iv) in this case. 
Suppose $\alpha \in(1 / 2)+\mathbb{Z}$. Now (2-19) implies that the multiplicity of

$$
\delta\left(\left[\nu^{1 / 2} \rho, \nu^{\alpha+n} \rho\right]\right)^{2} \otimes \sigma \text { in } s_{G L}(\pi)
$$

is $\geq 1$ (since $-\alpha-n \leq 1 / 2 \leq \alpha$ ). In this case, Lemma 2.2 and the exactness of Jacquet functors imply (iv) when $\alpha \in(1 / 2+\mathbb{Z})$.

Note that $\tilde{\pi}$ is a subquotient of $\delta\left(\left[\nu^{-\alpha-n} \rho, \nu^{\alpha+n} \rho\right]\right) \rtimes \tilde{\sigma}$ and $\delta\left(\left[\nu^{-\alpha+1} \rho, \nu^{\alpha+n} \rho\right]\right)^{\sim} \rtimes$ $\delta\left(\left[\nu^{\alpha} \rho, \nu^{\alpha+n} \rho\right], \sigma\right)^{\sim} \cong \delta\left(\left[\nu^{-\alpha-n} \rho, \nu^{\alpha-1} \rho\right]\right) \rtimes \delta\left(\left[\nu^{\alpha} \rho, \nu^{\alpha+n} \rho\right], \tilde{\sigma}\right)$. Since

$$
\delta\left(\left[\nu^{-\alpha-n} \rho, \nu^{\alpha-1} \rho\right]\right) \rtimes \delta\left(\left[\nu^{\alpha} \rho, \nu^{\alpha+n} \rho\right], \tilde{\sigma}\right)
$$

and

$$
\delta\left(\left[\nu^{-\alpha+1} \rho, \nu^{\alpha+n} \rho\right]\right) \rtimes \delta\left(\left[\nu^{\alpha} \rho, \nu^{\alpha+n} \rho\right], \tilde{\sigma}\right)
$$

have the same Jordan-Hölder series, the characterization of $\delta\left(\left[\nu^{-\alpha-n} \rho, \nu^{\alpha+n} \rho\right], \tilde{\sigma}\right)$ implies $\tilde{\pi} \cong \delta\left(\left[\nu^{-\alpha-n} \rho, \nu^{\alpha+n} \rho\right], \tilde{\sigma}\right)$. From $\tilde{\pi} \oplus \tilde{\pi}_{-} \cong \delta\left(\left[\nu^{-\alpha-n} \rho, \nu^{\alpha+n} \rho\right]\right) \rtimes \tilde{\sigma}=$ $\delta\left(\left[\nu^{-\alpha-n} \rho, \nu^{\alpha+n} \rho\right], \tilde{\sigma}\right) \oplus \delta\left(\left[\nu^{-\alpha-n} \rho, \nu^{\alpha+n} \rho\right]_{-}, \tilde{\sigma}\right)$ and $\tilde{\pi} \cong \delta\left(\left[\nu^{-\alpha-n} \rho, \nu^{\alpha+n} \rho\right], \tilde{\sigma}\right)$, we get $\tilde{\pi}_{-} \cong \delta\left(\left[\nu^{-\alpha-n} \rho, \nu^{\alpha+n} \rho\right]_{-}, \tilde{\sigma}\right)$. Now the proof of the theorem is complete.

\section{DeFinition of REPRESENTATIONS CORRESPONDING TO SEGMENTS}

In this section $\rho, \sigma$ and $\alpha$ will be as in the previous section: $\rho$ is an irreducible unitarizable cuspidal representation of $G L(p, F), \sigma$ is an irreducible cuspidal representation of $S_{q}$ and $\alpha \in(1 / 2) \mathbb{Z}, \alpha \geq 0$ is such that $\nu^{\alpha} \rho \rtimes \sigma$ reduces and $\nu^{\beta} \rho \rtimes \sigma$ is irreducible for $\beta \in(\alpha+\mathbb{Z}) \backslash\{ \pm \alpha\}$. Further, we shall fix $n, m \in \mathbb{Z}$ which satisfy $0 \leq n<m$.

First we shall write four formulas which we will need in the proof of the lemma that follows and later:

$$
\begin{aligned}
& \quad \mu^{*}\left(\delta\left(\left[\nu^{-\alpha-n} \rho, \nu^{\alpha+m} \rho\right]\right) \rtimes \sigma\right) \\
& =\sum_{a=-\alpha-n-1}^{\alpha+m} \sum_{b=a}^{\alpha+m} \delta\left(\left[\nu^{-a} \rho, \nu^{\alpha+n} \rho\right]\right) \times \delta\left(\left[\nu^{b+1} \rho, \nu^{\alpha+m} \rho\right]\right) \otimes \delta\left(\left[\nu^{a+1} \rho, \nu^{b} \rho\right]\right) \rtimes \sigma, \\
& \text { s.s. }\left(s_{G L}\left(\delta\left(\left[\nu^{-\alpha-n} \rho, \nu^{\alpha+m} \rho\right]\right) \rtimes \sigma\right)\right) \\
& =\sum_{i=-\alpha-n}^{\alpha+m+1} \delta\left(\left[\nu^{i} \rho, \nu^{\alpha+m} \rho\right]\right) \times \delta\left(\left[\nu^{-i+1} \rho, \nu^{\alpha+n} \rho\right]\right) \otimes \sigma,
\end{aligned}
$$

$$
\begin{aligned}
& \mu^{*}\left(\delta\left(\left[\nu^{\alpha+n+1} \rho, \nu^{\alpha+m} \rho\right]\right) \times \delta\left(\left[\nu^{-\alpha-n} \rho, \nu^{\alpha+n} \rho\right]\right) \rtimes \sigma\right) \\
& \quad=\sum_{a=\alpha+n}^{\alpha+m} \sum_{b=a}^{\alpha+m} \delta\left(\left[\nu^{-a} \rho, \nu^{-\alpha-n-1} \rho\right]\right) \times \delta\left(\left[\nu^{b+1} \rho, \nu^{\alpha+m} \rho\right]\right) \\
& \times \sum_{a^{\prime}=-\alpha-n-1}^{\alpha+n} \sum_{b^{\prime}=a^{\prime}}^{\alpha+n} \delta\left(\left[\nu^{-a^{\prime}} \rho, \nu^{\alpha+n} \rho\right]\right) \times \delta\left(\left[\nu^{b^{\prime}+1} \rho, \nu^{\alpha+n} \rho\right]\right) \\
& \otimes \delta\left(\left[\nu^{a+1} \rho, \nu^{b} \rho\right]\right) \times \delta\left(\left[\nu^{a^{\prime}+1} \rho, \nu^{b^{\prime}} \rho\right]\right) \rtimes \sigma,
\end{aligned}
$$




$$
\begin{gathered}
\text { s.s. }\left(s_{G L}\left(\delta\left(\left[\nu^{\alpha+n+1} \rho, \nu^{\alpha+m} \rho\right]\right) \times \delta\left(\left[\nu^{-\alpha-n} \rho, \nu^{\alpha+n} \rho\right]\right) \rtimes \sigma\right)\right) \\
=\left(\sum_{j=\alpha+n+1}^{\alpha+m+1} \delta\left(\left[\nu^{j} \rho, \nu^{\alpha+m} \rho\right]\right) \times \delta\left(\left[\nu^{-j+1} \rho, \nu^{-\alpha-n-1} \rho\right]\right)\right) \\
\times\left(\sum_{i=-\alpha-n}^{\alpha+n+1} \delta\left(\left[\nu^{i} \rho, \nu^{\alpha+n} \rho\right]\right) \times \delta\left(\left[\nu^{-i+1} \rho, \nu^{\alpha+n} \rho\right]\right)\right) \otimes \sigma .
\end{gathered}
$$

Note that (3-2) follows from (3-1), and (3-4) from (3-3). Theorems 2.3 and 2.5 imply

$$
\delta\left(\left[\nu^{-\alpha-n} \rho, \nu^{\alpha+n} \rho\right]\right) \rtimes \sigma=T_{1} \oplus T_{2},
$$

where $T_{1}$ and $T_{2}$ are inequivalent irreducible tempered representations.

3.1. Lemma. (i) The multiplicity of $\delta\left(\left[\nu^{-\alpha-n} \rho, \nu^{\alpha+m} \rho\right]\right) \otimes \sigma$ in $s_{G L}\left(\delta\left(\left[\nu^{-\alpha-n} \rho\right.\right.\right.$, $\left.\left.\left.\nu^{\alpha+m} \rho\right]\right) \rtimes \sigma\right)$ and in $s_{G L}\left(\delta\left(\left[\nu^{\alpha+n+1} \rho, \nu^{\alpha+m} \rho\right]\right) \times \delta\left(\left[\nu^{-\alpha-n} \rho, \nu^{\alpha+n} \rho\right]\right) \rtimes \sigma\right)$ is two.

(ii) The multiplicity of $\delta\left(\left[\nu^{\alpha+n+1} \rho, \nu^{\alpha+m} \rho\right]\right) \otimes T_{i}$ in $\mu^{*}\left(\delta\left(\left[\nu^{\alpha+n+1} \rho, \nu^{\alpha+m} \rho\right]\right) \rtimes T_{i}\right)$, in $\mu^{*}\left(\delta\left(\left[\nu^{\alpha+n+1} \rho, \nu^{\alpha+m} \rho\right]\right) \times \delta\left(\left[\nu^{-\alpha-n} \rho, \nu^{\alpha+n} \rho\right]\right) \rtimes \sigma\right)$ and in $\mu^{*}\left(\delta\left(\left[\nu^{-\alpha-n} \rho, \nu^{\alpha+m} \rho\right]\right)\right.$ $\rtimes \sigma)$ is one. Further, $\delta\left(\left[\nu^{\alpha+n+1} \rho, \nu^{\alpha+m} \rho\right]\right) \otimes T_{i}$ is not a subquotient of

$$
\mu^{*}\left(\delta\left(\left[\nu^{\alpha+n+1} \rho, \nu^{\alpha+m} \rho\right]\right) \rtimes T_{3-i}\right)
$$

(i.e., the multiplicity is 0 ).

Proof. Denote by $\pi=\delta\left(\left[\nu^{-\alpha-n} \rho, \nu^{\alpha+m} \rho\right]\right) \otimes \sigma$. First note that if $\pi$ is a subquotient of the $i$-th term of the sum in (3-2), then $i \geq-\alpha-n$ and $-i+1 \geq-\alpha-n$, since $\nu^{-\alpha-n-1} \rho$ is not in the $G L$-support of $\pi$. Further, since $\nu^{-\alpha-n} \rho$ is in the $G L$-support of $\pi$, we must have $i=-\alpha-n$ or $-i+1=-\alpha-n$, i.e. $i=-\alpha-n$ or $i=\alpha+n+1$. Now the $(-\alpha-n)$-th term in the sum (3-2) is just $\pi$. Further, the $(\alpha+n+1)$-th term is $\delta\left(\left[\nu^{\alpha+n+1} \rho, \nu^{\alpha+m} \rho\right]\right) \times \delta\left(\left[\nu^{-\alpha-n} \rho, \nu^{\alpha+n} \rho\right]\right) \otimes \sigma$. The multiplicity of $\pi$ in this term is one. Since $-\alpha-n \neq \alpha+n+1$, we have proved that the multiplicity of $\pi$ in (3-2) is two.

Now, we shall consider the multiplicity of $\pi$ in (3-4). Suppose that $\pi$ is a subquotient of the term of the double sum on the right-hand side of (3-4), which corresponds to indexes $j$ and $i$. Since $\nu^{-\alpha-n-1} \rho$ is not in the $G L$-support of $\pi$, we see that we must have $j=\alpha+n+1$. Note that for $-\alpha-n \leq i \leq \alpha+n+1$, $-\alpha-n \leq i$ and $-\alpha-n \leq-i+1$. Since $\nu^{-\alpha-n} \rho$ is in the $G L$-support of $\pi$, we must have $i=-\alpha-n$ or $-i+1=-\alpha-n$, i.e., $i=-\alpha-n$ or $i=\alpha+n+1$. For $j=\alpha+n+1$ and $i=-\alpha-n$ or $\alpha+n+1$, the corresponding term in both cases is $\delta\left(\left[\nu^{\alpha+n+1} \rho, \nu^{\alpha+m} \rho\right]\right) \times \delta\left(\left[\nu^{-\alpha-n} \rho, \nu^{\alpha+n} \rho\right]\right) \otimes \sigma$. The multiplicity of $\pi$ in this representation is one. Since $-\alpha-n \neq \alpha+n+1$, the multiplicity of $\pi$ in (3-4) is two. This ends the proof of (i).

Now we will study when we can have $\delta\left(\left[\nu^{\alpha+n+1} \rho, \nu^{\alpha+m} \rho\right]\right) \otimes T_{i}$ as a subquotient of the term of the right-hand side of (3-3) corresponding to indexes $a, a^{\prime}, b$ and $b^{\prime}$. Since $\nu^{-\alpha-n-1} \rho$ is not in the support of $\delta\left(\left[\nu^{\alpha+n+1} \rho, \nu^{\alpha+m} \rho\right]\right)$, from the term $\delta\left(\left[\nu^{-a} \rho, \nu^{-\alpha-n-1} \rho\right]\right)$ we see that we must have $-a>-\alpha-n-1$, i.e., $a<\alpha+n+1$ and further $a \leq \alpha+n$. Since $\alpha+n \leq a$, we get that it must be $a=\alpha+n$. Since $\nu^{\alpha+n} \rho$ is not in the support of $\delta\left(\left[\nu^{\alpha+n+1} \rho, \nu^{\alpha+m} \rho\right]\right)$, in the same way we conclude that we must have $-a^{\prime}>\alpha+n$, i.e., $a^{\prime}<-\alpha-n$. Since $-\alpha-n-1 \leq a^{\prime}$, $a^{\prime}=-\alpha-n-1$. For the same reason we must have $b^{\prime}+1>\alpha+n$. Now 
$b^{\prime} \leq \alpha+n$ implies $b^{\prime}=\alpha+n$. Therefore, up to now we have concluded that the term which has $\delta\left(\left[\nu^{\alpha+n+1} \rho, \nu^{\alpha+m} \rho\right]\right) \otimes T_{i}$ as a subquotient is $\delta\left(\left[\nu^{b+1} \rho, \nu^{\alpha+m} \rho\right]\right) \otimes$ $\delta\left(\left[\nu^{\alpha+n+1} \rho, \nu^{b} \rho\right]\right) \times \delta\left(\left[\nu^{-\alpha-n} \rho, \nu^{\alpha+n} \rho\right]\right) \rtimes \sigma$, with $\alpha+n \leq b \leq \alpha+m$. Since $\nu^{\alpha+n+1} \rho$ is in the support of $\delta\left(\left[\nu^{\alpha+n+1} \rho, \nu^{\alpha+m} \rho\right]\right)$, we need to have $b+1 \leq \alpha+n+1$. Now $\alpha+n \leq b$ implies $b=\alpha+n$. Therefore, there is only one set of indexes $a, a^{\prime}, b, b^{\prime}$ for which $\delta\left(\left[\nu^{\alpha+n+1} \rho, \nu^{\alpha+m} \rho\right]\right) \otimes T_{i}$ could be a subquotient. Then the corresponding term is $\delta\left(\left[\nu^{\alpha+n+1} \rho, \nu^{\alpha+m} \rho\right]\right) \otimes \delta\left(\left[\nu^{-\alpha-n} \rho, \nu^{\alpha+n} \rho\right]\right) \rtimes \sigma$. From (3-5) we know that the multiplicity of $\delta\left(\left[\nu^{\alpha+n+1} \rho, \nu^{\alpha+m} \rho\right]\right) \otimes T_{i}$ in this term is one. This completes the proof that the multiplicity of $\delta\left(\left[\nu^{\alpha+n+1} \rho, \nu^{\alpha+m} \rho\right]\right) \otimes T_{i}$ in $(3-3)$ is one.

Frobenius reciprocity implies

$$
\delta\left(\left[\nu^{\alpha+n+1} \rho, \nu^{\alpha+m} \rho\right]\right) \otimes T_{i} \leq \mu^{*}\left(\delta\left(\left[\nu^{\alpha+n+1} \rho, \nu^{\alpha+m} \rho\right]\right) \rtimes T_{i}\right) .
$$

Now $\delta\left(\left[\nu^{\alpha+n+1} \rho, \nu^{\alpha+m} \rho\right]\right) \rtimes\left(\delta\left(\left[\nu^{-\alpha-n} \rho, \nu^{\alpha+n} \rho\right]\right) \rtimes \sigma\right)=\delta\left(\left[\nu^{\alpha+n+1} \rho, \nu^{\alpha+m} \rho\right]\right) \rtimes T_{1} \oplus$ $\delta\left(\left[\nu^{\alpha+n+1} \rho, \nu^{\alpha+m} \rho\right]\right) \rtimes T_{2}$ and the first part of the proof imply that the multiplicity of $\delta\left(\left[\nu^{\alpha+n+1} \rho, \nu^{\alpha+m} \rho\right]\right) \otimes T_{i}$ in $\mu^{*}\left(\delta\left(\left[\nu^{\alpha+n+1} \rho, \nu^{\alpha+m} \rho\right]\right) \rtimes T_{i}\right)$ and $\mu^{*}\left(\delta\left(\left[\nu^{\alpha+n+1} \rho\right.\right.\right.$, $\left.\left.\left.\nu^{\alpha+m} \rho\right]\right) \rtimes T_{3-i}\right)$ is one and zero, respectively.

Note that the term in (3-1) corresponding to $a=-\alpha-n-1, b=\alpha+n$ is the representation $\delta\left(\left[\nu^{\alpha+n+1} \rho, \nu^{\alpha+m} \rho\right]\right) \otimes \delta\left(\left[\nu^{-\alpha-n} \rho, \nu^{\alpha+n} \rho\right]\right) \rtimes \sigma$. This implies $\delta\left(\left[\nu^{\alpha+n+1} \rho, \nu^{\alpha+m} \rho\right]\right) \otimes T_{i} \leq \mu^{*}\left(\delta\left(\left[\nu^{-\alpha-n} \rho, \nu^{\alpha+m} \rho\right]\right) \rtimes \sigma\right)$. Since $\delta\left(\left[\nu^{-\alpha-n} \rho, \nu^{\alpha+m} \rho\right]\right) \rtimes$ $\sigma \leq \delta\left(\left[\nu^{\alpha+n+1} \rho, \nu^{\alpha+m} \rho\right]\right) \times \delta\left(\left[\nu^{-\alpha-n} \rho, \nu^{\alpha+n} \rho\right]\right) \rtimes \sigma$, the first part of the proof implies that $\delta\left(\left[\nu^{\alpha+n+1} \rho, \nu^{\alpha+m} \rho\right]\right) \otimes T_{i}$ has multiplicity one in $\mu^{*}\left(\delta\left(\left[\nu^{-\alpha-n} \rho, \nu^{\alpha+m} \rho\right]\right) \rtimes \sigma\right)$. Now the proof of the lemma is complete.

3.2. Proposition. Let $\rho$ and $\sigma$ be irreducible unitarizable cuspidal representations of $G L(p, F)$ and $S_{q}$ respectively, and let $\alpha \in(1 / 2) \mathbb{Z}, \alpha \geq 0$. Suppose that $\nu^{\alpha} \rho \rtimes \sigma$ reduces and $\nu^{\beta} \rho \rtimes \sigma$ is irreducible for $\beta \in(\alpha+\mathbb{Z}) \backslash\{ \pm \alpha\}$. Let $n, m \in \mathbb{Z}, m>n \geq 0$. Suppose that $T_{1}$ and $T_{2}$ satisfy (3-5).

(i) The representation $\delta\left(\left[\nu^{\alpha+n+1} \rho, \nu^{\alpha+m} \rho\right]\right) \rtimes T_{i}$ has a unique irreducible subrepresentation, which will be denoted by

$$
\delta\left(\left[\nu^{-\alpha-n} \rho, \nu^{\alpha+m} \rho\right], \sigma\right)_{T_{i}}
$$

Representations $\delta\left(\left[\nu^{-\alpha-n} \rho, \nu^{\alpha+m} \rho\right], \sigma\right)_{T_{1}}$ and $\delta\left(\left[\nu^{-\alpha-n} \rho, \nu^{\alpha+m} \rho\right], \sigma\right)_{T_{2}}$ are inequivalent. They are subrepresentations of $\delta\left(\left[\nu^{-\alpha-n} \rho, \nu^{\alpha+m} \rho\right]\right) \rtimes \sigma$ and they are the only irreducible subrepresentations of it.

(ii) $\delta\left(\left[\nu^{-\alpha-n} \rho, \nu^{\alpha+m} \rho\right], \sigma\right)_{T_{i}}$ is a unique irreducible subquotient of $\delta\left(\left[\nu^{\alpha+n+1} \rho\right.\right.$, $\left.\left.\nu^{\alpha+m} \rho\right]\right) \rtimes T_{i}$ which has $\delta\left(\left[\nu^{-\alpha-n} \rho, \nu^{\alpha+m} \rho\right]\right) \otimes \sigma$ for a subquotient of a corresponding Jacquet module.

(iii) $\delta\left(\left[\nu^{-\alpha-n} \rho, \nu^{\alpha+m} \rho\right], \sigma\right)_{T_{i}}^{\sim} \cong \delta\left(\left[\nu^{-\alpha-n} \rho, \nu^{\alpha+m} \rho\right], \tilde{\sigma}\right)_{\tilde{T}_{i}}$.

Proof. By Frobenius reciprocity, $\delta\left(\left[\nu^{\alpha+n+1} \rho, \nu^{\alpha+m} \rho\right]\right) \otimes T_{i} \leq \mu^{*}(\tau)$ for each irreducible subrepresentation $\tau$ of $\delta\left(\left[\nu^{\alpha+n+1} \rho, \nu^{\alpha+m} \rho\right]\right) \rtimes T_{i}$. The above lemma shows that the multiplicity of $\delta\left(\left[\nu^{\alpha+n+1} \rho, \nu^{\alpha+m} \rho\right]\right) \otimes T_{i}$ in $\mu^{*}\left(\delta\left(\left[\nu^{\alpha+n+1} \rho, \nu^{\alpha+m} \rho\right]\right) \rtimes T_{i}\right)$ is one. This implies the uniqueness of irreducible subrepresentation in $\delta\left(\left[\nu^{\alpha+n+1} \rho\right.\right.$, $\left.\left.\nu^{\alpha+m} \rho\right]\right) \rtimes T_{i}$.

Denote $\pi_{i}=\delta\left(\left[\nu^{\alpha+n+1} \rho, \nu^{\alpha+m} \rho\right], \sigma\right)_{T_{i}}$. As we noted, $\delta\left(\left[\nu^{\alpha+n+1} \rho, \nu^{\alpha+m} \rho\right]\right) \otimes T_{i} \leq$ $\mu^{*}\left(\pi_{i}\right)$. Lemma 3.1 (ii) implies $\delta\left(\left[\nu^{\alpha+n+1} \rho, \nu^{\alpha+m} \rho\right]\right) \otimes T_{3-i} \not \leq \mu^{*}\left(\pi_{i}\right)$. This implies inequivalence of $\pi_{1}$ and $\pi_{2}$. 
Consider now

$$
\begin{aligned}
& \delta\left(\left[\nu^{-\alpha-n} \rho, \nu^{\alpha+m} \rho\right]\right) \rtimes \sigma \hookrightarrow \delta\left(\left[\nu^{\alpha+n+1} \rho, \nu^{\alpha+m} \rho\right]\right) \times \delta\left(\left[\nu^{-\alpha-n} \rho, \nu^{\alpha+n} \rho\right]\right) \rtimes \sigma \\
& \cong \delta\left(\left[\nu^{\alpha+n+1} \rho, \nu^{\alpha+m} \rho\right]\right) \rtimes T_{1} \oplus \delta\left(\left[\nu^{\alpha+n+1} \rho, \nu^{\alpha+m} \rho\right]\right) \rtimes T_{2} .
\end{aligned}
$$

We shall identify $\delta\left(\left[\nu^{-\alpha-n} \rho, \nu^{\alpha+m} \rho\right]\right) \rtimes \sigma$ with a subrepresentation of (3-7) under the embedding in (3-6) and the isomorphism in (3-7). Note that (ii) of Lemma 3.1 and the above paragraph imply that the multiplicity of $\pi_{i}$ in (3-7) is one. Suppose $\pi_{i} \cap$ $\delta\left(\left[\nu^{-\alpha-n} \rho, \nu^{\alpha+m} \rho\right]\right) \rtimes \sigma=\{0\}$. Since the multiplicity of $\delta\left(\left[\nu^{\alpha+n+1} \rho, \nu^{\alpha+m} \rho\right]\right) \otimes T_{i}$ in $\mu^{*}\left(\pi_{i}\right)$ and $\mu^{*}\left(\delta\left(\left[\nu^{-\alpha-n} \rho, \nu^{\alpha+m} \rho\right]\right) \rtimes \sigma\right)$ is one (the later multiplicity follows from (ii) of Lemma 3.1), the exactness of Jacquet functors implies that the multiplicity of the representation $\delta\left(\left[\nu^{\alpha+n+1} \rho, \nu^{\alpha+m} \rho\right]\right) \otimes T_{i}$ in the corresponding Jacquet module of (3-7) is $\geq 2$. This contradicts (ii) of Lemma 3.1. Thus, $\pi_{i}$ are irreducible subrepresentations of $\delta\left(\left[\nu^{-\alpha-n} \rho, \nu^{\alpha+m} \rho\right]\right) \rtimes \sigma$. Since $\delta\left(\left[\nu^{-\alpha-n} \rho, \nu^{\alpha+m} \rho\right]\right) \otimes \sigma \leq s_{G L}(\tau)$ for each irreducible subrepresentation $\tau$ of $\delta\left(\left[\nu^{-\alpha-n} \rho, \nu^{\alpha+m} \rho\right]\right) \rtimes \sigma$, and the multiplicity of $\delta\left(\left[\nu^{-\alpha-n} \rho, \nu^{\alpha+m} \rho\right]\right) \otimes \sigma$ in $s_{G L}\left(\delta\left(\left[\nu^{-\alpha-n} \rho, \nu^{\alpha+m} \rho\right]\right) \rtimes \sigma\right)$ is two by (i) of Lemma 3.1, $\delta\left(\left[\nu^{-\alpha-n} \rho, \nu^{\alpha+m} \rho\right]\right) \rtimes \sigma$ has at most two irreducible subrepresentations. Therefore, $\pi_{i}$ are the only irreducible subrepresentations of $\delta\left(\left[\nu^{-\alpha-n} \rho, \nu^{\alpha+m} \rho\right]\right) \rtimes \sigma$. Note that $\delta\left(\left[\nu^{-\alpha-n} \rho, \nu^{\alpha+m} \rho\right]\right) \otimes \sigma \leq s_{G L}\left(\pi_{i}\right)$ implies $\delta\left(\left[\nu^{-\alpha-n} \rho, \nu^{\alpha+m} \rho\right]\right) \otimes \sigma \leq$ $s_{G L}\left(\delta\left(\left[\nu^{\alpha+n+1} \rho, \nu^{\alpha+m} \rho\right]\right) \rtimes T_{i}\right)$. Now (i) of Lemma 3.1 implies that the multiplicity of $\delta\left(\left[\nu^{-\alpha-n} \rho, \nu^{\alpha+m} \rho\right]\right) \otimes \sigma$ in $s_{G L}\left(\delta\left(\left[\nu^{\alpha+n+1} \rho, \nu^{\alpha+m} \rho\right]\right) \rtimes T_{i}\right)$ is one. This and $\delta\left(\left[\nu^{-\alpha-n} \rho, \nu^{\alpha+m} \rho\right]\right) \otimes \sigma \leq s_{G L}\left(\pi_{i}\right)$ imply the characterization of $\pi_{i}$ in (ii).

Note that $\delta\left(\left[\nu^{-\alpha-n} \rho, \nu^{\alpha+n} \rho\right]\right) \rtimes \tilde{\sigma} \cong \tilde{T}_{1} \oplus \tilde{T}_{2}$. Observe that $\tilde{\pi}_{i}$ is a quotient of the representation $\delta\left(\left[\nu^{-\alpha-m} \rho, \nu^{-\alpha-n-1} \rho\right]\right) \rtimes \tilde{T}_{i}$. The reciprocity (2) in Theorem 2.4 .3 of $[\mathrm{Si}]$ implies that $\delta\left(\left[\nu^{-\alpha-m} \rho, \nu^{-\alpha-n-1} \rho\right]\right) \otimes \tilde{T}_{i}$ is a quotient of a Jacquet module of $\tilde{\pi}_{i}$ with respect to a corresponding parabolic subgroup which is opposite to a standard one. Conjugating the Jacquet modules with an element of the Weyl group which carry the opposite parabolic subgroup to the standard one, we get $\delta\left(\left[\nu^{\alpha+n+1} \rho, \nu^{\alpha+m} \rho\right]\right) \otimes \tilde{T}_{i} \leq \mu^{*}\left(\tilde{\pi}_{i}\right)$. This implies (iii).

We shall now introduce a convention which will simplify notation in the rest of the paper.

Suppose $\alpha=0$. Write $\rho \rtimes \sigma=\tau_{1} \oplus \tau_{2}$ as a sum of irreducible representations. In Theorem 2.3 we have introduced representations $\delta\left(\left[\nu^{-\alpha-n} \rho, \nu^{\alpha+n} \rho\right]_{\tau_{i}}, \sigma\right)$. For $T_{i}=\delta\left(\left[\nu^{-\alpha-n} \rho, \nu^{\alpha+n} \rho\right]_{\tau_{i}}, \sigma\right)$ we shall denote $\delta\left(\left[\nu^{-\alpha-n} \rho, \nu^{\alpha+m} \rho\right], \sigma\right)_{T_{i}}$ also by

$$
\delta\left(\left[\nu^{-\alpha-n} \rho, \nu^{\alpha+m} \rho\right]_{\tau_{i}}, \sigma\right) .
$$

Now we get $\delta\left(\left[\nu^{-\alpha-n} \rho, \nu^{\alpha+m} \rho\right]_{\tau_{i}}, \sigma\right)^{\sim} \cong \delta\left(\left[\nu^{-\alpha-n} \rho, \nu^{\alpha+m} \rho\right]_{\tilde{\tau}_{i}}, \tilde{\sigma}\right)$ from Theorem 2.3 and Proposition 3.2.

Let $\alpha>0$. In Theorem 2.5 we have introduced representations $\delta\left(\left[\nu^{-\alpha-n} \rho\right.\right.$, $\left.\left.\nu^{\alpha+n} \rho\right]_{ \pm}, \sigma\right)$. We shall denote $\delta\left(\left[\nu^{-\alpha-n} \rho, \nu^{\alpha+m} \rho\right], \sigma\right)_{\delta\left(\left[\nu^{-\alpha-n} \rho, \nu^{\alpha+n} \rho\right]_{ \pm}, \sigma\right)}$ also by

$$
\delta\left(\left[\nu^{-\alpha-n} \rho, \nu^{\alpha+m} \rho\right]_{ \pm}, \sigma\right) .
$$

We shall also denote $\delta\left(\left[\nu^{-\alpha-n} \rho, \nu^{\alpha+m} \rho\right]_{+}, \sigma\right)$ simply by $\delta\left(\left[\nu^{-\alpha-n} \rho, \nu^{\alpha+m} \rho\right], \sigma\right)$. Theorem 2.5 and Proposition 3.2 imply $\delta\left(\left[\nu^{-\alpha-n} \rho, \nu^{\alpha+m} \rho\right]_{ \pm}, \sigma\right)^{\sim} \cong \delta\left(\left[\nu^{-\alpha-n} \rho\right.\right.$, $\left.\left.\nu^{\alpha+m} \rho\right]_{ \pm}, \tilde{\sigma}\right)$.

We shall end this section with a simple lemma. 
3.3. Lemma. The multiplicity of $\delta\left(\left[\nu^{\epsilon(\alpha)} \rho, \nu^{\alpha+m} \rho\right]\right) \times \delta\left(\left[\nu^{1-\epsilon(\alpha)} \rho, \nu^{\alpha+n} \rho\right]\right) \otimes \sigma$ in the representations $s_{G L}\left(\delta\left(\left[\nu^{-\alpha-n} \rho, \nu^{\alpha+m} \rho\right]\right) \rtimes \sigma\right)$ and $s_{G L}\left(\left(\prod_{i=-\alpha-n}^{\alpha+m} \nu^{i} \rho\right) \rtimes \sigma\right)$ is $2(1-\epsilon(\alpha))$.

Proof. First note s.s. $\left(s_{G L}\left(\left(\prod_{i=-\alpha-n}^{\alpha+m} \nu^{i} \rho\right) \rtimes \sigma\right)\right)=\sum\left(\prod_{i=-\alpha-n}^{\alpha+m} \nu^{\epsilon_{i} i} \rho\right) \otimes \sigma$, where the sum runs over all $\epsilon_{-\alpha-n}, \epsilon_{-\alpha-n+1}, \ldots, \epsilon_{\alpha+m} \in\{ \pm 1\}$ (one gets this formula in the same way as (2-10); the proof of the lemma is also similar to the proof of Lemma 2.2).

Let $\alpha \in \mathbb{Z}$. To get $\delta\left(\left[\rho, \nu^{\alpha+m} \rho\right]\right) \times \delta\left(\left[\nu \rho, \nu^{\alpha+n} \rho\right]\right) \otimes \sigma$ as a subquotient of the above representation, we must have all $\epsilon_{i} i \geq$. Obviously, only two systems of $\epsilon_{i}$ 's satisfy this condition. The multiplicity in the corresponding terms is one. This directly implies the multiplicity two of $\delta\left(\left[\rho, \nu^{\alpha+m} \rho\right]\right) \times \delta\left(\left[\nu \rho, \nu^{\alpha+n} \rho\right]\right) \otimes \sigma$ in the above representation.

Considering $G L$-supports, we see that we can get $\delta\left(\left[\rho, \nu^{\alpha+m} \rho\right]\right) \times \delta\left(\left[\nu \rho, \nu^{\alpha+n} \rho\right]\right) \otimes$ $\sigma$ as a subquotient of a term in the sum (3-2) only if $i=0$ or 1. For corresponding terms the multiplicity is one. Therefore, the multiplicity of $\delta\left(\left[\rho, \nu^{\alpha+m} \rho\right]\right) \times$ $\delta\left(\left[\nu \rho, \nu^{\alpha+n} \rho\right]\right) \otimes \sigma$ in (3-2) is two. This completes the proof of the lemma for $\alpha \in \mathbb{Z}$.

Suppose $\alpha \in(1 / 2+\mathbb{Z})$. In this case, only one system of $\epsilon_{i}$ 's satisfy $\epsilon_{i} i>$ 0 (since $i \in 1 / 2+\mathbb{Z})$. This implies the multiplicity one of $\delta\left(\left[\nu^{1 / 2} \rho, \nu^{\alpha+m} \rho\right]\right) \times$ $\delta\left(\left[\nu^{1 / 2} \rho, \nu^{\alpha+n} \rho\right]\right) \otimes \sigma$ in the Jacquet module $s_{G L}\left(\left(\prod_{i=-\alpha-n}^{\alpha+m} \nu^{i} \rho\right) \rtimes \sigma\right)$. Further, note that the representation $\delta\left(\left[\nu^{1 / 2} \rho, \nu^{\alpha+m} \rho\right]\right) \times \delta\left(\left[\nu^{1 / 2} \rho, \nu^{\alpha+n} \rho\right]\right) \otimes \sigma$ is equal to the term of the sum (3-2) corresponding to $i=1 / 2$, and all other terms in the sum (3-2) have different $G L$-supports from the $G L$-support of $\delta\left(\left[\nu^{1 / 2} \rho, \nu^{\alpha+m} \rho\right]\right) \times$ $\delta\left(\left[\nu^{1 / 2} \rho, \nu^{\alpha+n} \rho\right]\right) \otimes \sigma$. This implies the multiplicity one of the last representation in (3-2). The proof is now complete.

\section{Square integrability, The CASE OF POSItive $\alpha$}

In this section $\rho, \sigma, \alpha, n$ and $m$ are as in the last section, except that we additionally assume, in this section that, $\alpha>0$.

4.1. Lemma. Let $\pi$ be an irreducible subquotient of $\left(\prod_{i=-\alpha-n}^{\alpha+m} \nu^{i} \rho\right) \rtimes \sigma$ such that $\delta\left(\left[\nu^{\epsilon(\alpha)} \rho, \nu^{\alpha+m} \rho\right]\right) \times \delta\left(\left[\nu^{1-\epsilon(\alpha)} \rho, \nu^{\alpha+n} \rho\right]\right) \otimes \sigma \leq s_{G L}(\pi)$. Then

(i) $\pi \leq \delta\left(\left[\nu^{-\alpha-n} \rho, \nu^{\alpha+m} \rho\right]\right) \rtimes \sigma$;

(ii) $\delta\left(\left[\nu^{-\alpha-n} \rho, \nu^{\alpha+m} \rho\right]\right) \otimes \sigma \leq s_{G L}(\pi)$;

(iii) $\pi \cong \delta\left(\left[\nu^{-\alpha-n} \rho, \nu^{\alpha+m} \rho\right], \sigma\right)$;

(iv) $2 \delta\left(\left[\rho, \nu^{\alpha+m} \rho\right]\right) \times \delta\left(\left[\nu \rho, \nu^{\alpha+n} \rho\right]\right) \otimes \sigma \leq s_{G L}(\pi)$ if $\alpha \in \mathbb{Z}$.

Proof. Lemma 3.3 and the exactness of the Jacquet functor imply (i).

Suppose $\alpha \in \mathbb{Z}$. One directly checks that

$$
\begin{aligned}
& 2 \delta\left(\left[\rho, \nu^{\alpha+m} \rho\right]\right) \times \delta\left(\left[\nu \rho, \nu^{\alpha+n} \rho\right]\right) \otimes \sigma \\
& \quad \leq \text { s.s. }\left(s_{G L}\left(\delta\left(\left[\nu^{-\alpha-n} \rho, \nu^{\alpha-1} \rho\right]\right) \rtimes \delta\left(\left[\nu^{\alpha} \rho, \nu^{\alpha+m} \rho\right], \sigma\right)\right)\right) \\
& \quad=\delta\left(\left[\nu^{\alpha} \rho, \nu^{\alpha+m} \rho\right]\right) \times \sum_{i=-\alpha-n}^{\alpha} \delta\left(\left[\nu^{i} \rho, \nu^{\alpha-1} \rho\right]\right) \times \delta\left(\left[\nu^{-i+1} \rho, \nu^{\alpha+n} \rho\right], \sigma\right) \otimes \sigma
\end{aligned}
$$

(consider the terms in the sum for $i=0$ and 1). Observe that (4-1) implies

$$
\delta\left(\left[\nu^{-\alpha-n} \rho, \nu^{\alpha+m} \rho\right]\right) \otimes \sigma \leq s_{G L}\left(\delta\left(\left[\nu^{-\alpha-n} \rho, \nu^{\alpha-1} \rho\right]\right) \rtimes \delta\left(\left[\nu^{\alpha} \rho, \nu^{\alpha+m} \rho\right], \sigma\right)\right) .
$$


Further, (4-1) and Lemma 3.3 imply

$$
\pi \leq \delta\left(\left[\nu^{-\alpha-n} \rho, \nu^{\alpha-1} \rho\right]\right) \rtimes \delta\left(\left[\nu^{\alpha} \rho, \nu^{\alpha+m} \rho\right], \sigma\right) .
$$

The assumption on $\pi$ and (1-4) imply

$$
\delta\left(\left[\rho, \nu^{\alpha+m} \rho\right]\right) \times \delta\left(\left[\nu \rho, \nu^{\alpha+m} \rho\right]\right) \otimes \sigma \leq s_{G L}\left(\delta\left(\left[\nu^{\alpha+n+1} \rho, \nu^{\alpha+m} \rho\right]\right) \rtimes \pi\right) .
$$

Now (iv) of Theorem 2.5 implies

$$
\delta\left(\left[\nu^{-\alpha-m} \rho, \nu^{\alpha+m} \rho\right], \sigma\right) \leq \delta\left(\left[\nu^{\alpha+n+1} \rho, \nu^{\alpha+m} \rho\right]\right) \rtimes \pi .
$$

Further, (iii) of the same theorem implies

$$
\delta\left(\left[\nu^{-\alpha-m} \rho, \nu^{\alpha+m} \rho\right]\right) \otimes \sigma \leq s_{G L}\left(\delta\left(\left[\nu^{\alpha+n+1} \rho, \nu^{\alpha+m} \rho\right]\right) \rtimes \pi\right) .
$$

Suppose $\pi \neq \delta\left(\left[\nu^{-\alpha-n} \rho, \nu^{\alpha+m} \rho\right]_{ \pm}, \sigma\right)$. Then (i) of Lemma 4.1, (i) of Proposition 3.2 and (i) of Lemma 3.1 imply that $\delta\left(\left[\nu^{-\alpha-n} \rho, \nu^{\alpha+m} \rho\right]\right) \otimes \sigma$ is not a subquotient of $s_{G L}(\pi)$. Further, (4-3) and (4-2) imply

$$
s_{G L}(\pi) \leq s_{G L}\left(\delta\left(\left[\nu^{-\alpha-n} \rho, \nu^{\alpha-1} \rho\right]\right) \rtimes \delta\left(\left[\nu^{\alpha} \rho, \nu^{\alpha+m} \rho\right], \sigma\right)\right)-\delta\left(\left[\nu^{-\alpha-n} \rho, \nu^{\alpha+m} \rho\right]\right) \otimes \sigma .
$$

This and (4-6) imply

$$
\begin{aligned}
& \delta\left(\left[\nu^{-\alpha-m} \rho, \nu^{\alpha+m} \rho\right]\right) \otimes \sigma \leq \sum_{i=\alpha+n+1}^{\alpha+m+1} \delta\left(\left[\nu^{i} \rho, \nu^{\alpha+m} \rho\right]\right) \\
& \times \delta\left(\left[\nu^{-i+1} \rho, \nu^{-\alpha-n-1} \rho\right]\right) \\
& \times\left(\delta\left(\left[\nu^{\alpha} \rho, \nu^{\alpha+m} \rho\right]\right) \times \sum_{j=-\alpha+1}^{\alpha+n+1} \delta\left(\left[\nu^{j} \rho, \nu^{\alpha+n} \rho\right]\right)\right. \\
& \left.\quad \times \delta\left(\left[\nu^{-j+1} \rho, \nu^{\alpha-1} \rho\right]\right)-\delta\left(\left[\nu^{-\alpha-n} \rho, \nu^{\alpha+m} \rho\right]\right)\right) \otimes \sigma .
\end{aligned}
$$

Note that $\nu^{-\alpha-m} \rho$ and $\nu^{-\alpha-n} \rho$ are in the $G L$-support of $\delta\left(\left[\nu^{-\alpha-n} \rho, \nu^{\alpha+m} \rho\right]\right) \otimes \sigma$. It is easy to see that the only term in the above sum, which has both representations in the $G L$-support, is the term corresponding to $i=\alpha+m+1$ and $j=\alpha+n+1$ (use that $-\alpha-m<\alpha+n+1$ and $-\alpha-n<-\alpha+1$ ). Thus

$$
\begin{aligned}
& \delta\left(\left[\nu^{-\alpha-m} \rho, \nu^{\alpha+m} \rho\right]\right) \leq \delta\left(\left[\nu^{-\alpha-m} \rho, \nu^{-\alpha-n-1} \rho\right]\right) \\
& \quad \times\left(\delta\left(\left[\nu^{\alpha} \rho, \nu^{\alpha+m} \rho\right]\right) \times \delta\left(\left[\nu^{-\alpha-n} \rho, \nu^{\alpha-1} \rho\right]\right)-\delta\left(\left[\nu^{-\alpha-n} \rho, \nu^{\alpha+m} \rho\right]\right)\right) \\
& =\delta\left(\left[\nu^{-\alpha-m} \rho, \nu^{-\alpha-n-1} \rho\right]\right) \times \delta\left(\left[\nu^{\alpha} \rho, \nu^{\alpha+m} \rho\right]\right) \times \delta\left(\left[\nu^{-\alpha-n} \rho, \nu^{\alpha-1} \rho\right]\right) \\
& \left.\quad-\delta\left(\left[\nu^{-\alpha-m} \rho, \nu^{-\alpha-n-1} \rho\right]\right) \times \delta\left(\left[\nu^{-\alpha-n} \rho, \nu^{\alpha+m} \rho\right]\right)\right) .
\end{aligned}
$$

Since the multiplicity of $\delta\left(\left[\nu^{-\alpha-m} \rho, \nu^{\alpha+m} \rho\right]\right)$ in each of the representations in the last two lines of (4-8) is one, we get that (4-8) cannot hold. This contradiction implies $\pi \cong \delta\left(\left[\nu^{-\alpha-n} \rho, \nu^{\alpha+m} \rho\right]_{ \pm}, \sigma\right)$. This and (i) of Proposition 3.2 imply (ii) when $\alpha \in \mathbb{Z}$.

From (2-20) and (1-4) we see that $\delta\left(\left[\rho, \nu^{\alpha+m} \rho\right]\right) \times \delta\left(\left[\nu \rho, \nu^{\alpha+n} \rho\right]\right) \otimes \sigma$ is not a subquotient of

$$
\delta\left(\left[\nu^{\alpha+n+1} \rho, \nu^{\alpha+m} \rho\right]\right) \times \delta\left(\left[\nu^{-\alpha-n} \rho, \nu^{\alpha+n} \rho\right]_{-}, \sigma\right) .
$$

This implies $\pi \nsucceq \delta\left(\left[\nu^{-\alpha-n} \rho, \nu^{\alpha+m} \rho\right]_{-}, \sigma\right)$, which implies $\pi \cong \delta\left(\left[\nu^{-\alpha-n} \rho, \nu^{\alpha+m} \rho\right], \sigma\right)$. Thus, we have proved (iii) also, when $\alpha \in \mathbb{Z}$.

The case of $\alpha \in(1 / 2)+\mathbb{Z}$ proceeds analogously. One needs to check only that the inequality obtained from (4-1) putting $\delta\left(\left[\nu^{1 / 2} \rho, \nu^{\alpha+m} \rho\right]\right) \times \delta\left(\left[\nu^{1 / 2} \rho, \nu^{\alpha+n} \rho\right]\right) \otimes \sigma$ 
instead of $2 \delta\left(\left[\rho, \nu^{\alpha+m} \rho\right]\right) \times \delta\left(\left[\nu \rho, \nu^{\alpha+n} \rho\right]\right) \otimes \sigma$ holds (consider the term corresponding to $i=1 / 2)$, and that $\delta\left(\left[\nu^{1 / 2} \rho, \nu^{\alpha+m} \rho\right]\right) \times \delta\left(\left[\nu^{1 / 2} \rho, \nu^{\alpha+n} \rho\right]\right) \otimes \sigma$ is not a subquotient of (4-9), which follows from (2-20).

Let $\alpha \in \mathbb{Z}$. Suppose that the multiplicity of $\delta\left(\left[\rho, \nu^{\alpha+m} \rho\right]\right) \times \delta\left(\left[\nu \rho, \nu^{\alpha+n} \rho\right]\right) \otimes$ $\sigma$ in $s_{G L}(\pi)$ is one. Then (i) of Lemma 3.1 implies that there is an irreducible subquotient $\pi^{\prime}$ of $\delta\left(\left[\nu^{-\alpha-n} \rho, \nu^{\alpha+m} \rho\right]\right) \rtimes \sigma$ such that

$$
\begin{gathered}
\pi+\pi^{\prime} \leq \delta\left(\left[\nu^{-\alpha-n} \rho, \nu^{\alpha+m} \rho\right]\right) \rtimes \sigma, \\
\delta\left(\left[\rho, \nu^{\alpha+m} \rho\right]\right) \times \delta\left(\left[\nu \rho, \nu^{\alpha+n} \rho\right]\right) \otimes \sigma \leq s_{G L}\left(\pi^{\prime}\right) .
\end{gathered}
$$

Since $\pi^{\prime}$ satisfies the same condition (4-9) which is satisfied by $\pi$, then (4-5) also holds for $\pi^{\prime}$.

Multiplying relation (4-10) with $\delta\left(\left[\nu^{-\alpha-m} \rho, \nu^{-\alpha-n-1} \rho\right]\right)$ from the left-hand side, we get $\delta\left(\left[\nu^{-\alpha-m} \rho, \nu^{-\alpha-n-1} \rho\right]\right) \rtimes \pi+\delta\left(\left[\nu^{-\alpha-m} \rho, \nu^{-\alpha-n-1} \rho\right]\right) \rtimes \pi^{\prime} \leq \delta\left(\left[\nu^{-\alpha-m} \rho\right.\right.$, $\left.\left.\nu^{-\alpha-n-1} \rho\right]\right) \times \delta\left(\left[\nu^{-\alpha-n} \rho, \nu^{\alpha+m} \rho\right]\right) \rtimes \sigma$. This relation and inequalities (4-5) in the cases of $\pi$ and $\pi^{\prime}$, imply $2 \delta\left(\left[\nu^{-\alpha-m} \rho, \nu^{\alpha+m} \rho\right], \sigma\right) \leq \delta\left(\left[\nu^{-\alpha-m} \rho, \nu^{-\alpha-n-1} \rho\right]\right) \times$ $\delta\left(\left[\nu^{-\alpha-n} \rho, \nu^{\alpha+m} \rho\right]\right) \rtimes \sigma$. This contradicts to (iv) of Theorem 2.5, and ends the proof of (iv).

Summing up, it is easy to see that the following proposition holds.

4.2. Proposition. Let $\pi$ be an irreducible subquotient of $\left(\prod_{i=-\alpha-n}^{\alpha+m} \nu^{i} \rho\right) \rtimes \sigma$ which satisfies $\delta\left(\left[\nu^{\epsilon(\alpha)} \rho, \nu^{\alpha+m} \rho\right]\right) \times \delta\left(\left[\nu^{1-\epsilon(\alpha)} \rho, \nu^{\alpha+n} \rho\right]\right) \otimes \sigma \leq s_{G L}(\pi)$. Then the multiplicity of $\pi$ in $\left(\prod_{i=-\alpha-n}^{\alpha+m} \nu^{i} \rho\right) \rtimes \sigma$ is one, $\pi$ is a subquotient of $\delta\left(\left[\nu^{-\alpha-n} \rho, \nu^{\alpha+m} \rho\right]\right) \rtimes \sigma$ and $\pi \cong \delta\left(\left[\nu^{-\alpha-n} \rho, \nu^{\alpha+m} \rho\right], \sigma\right)$. Further, the multiplicity of $\delta\left(\left[\nu^{-\alpha-n} \rho, \nu^{\alpha+m} \rho\right]\right) \otimes \sigma$ in $s_{G L}(\pi)$ is one, and of $\delta\left(\left[\nu^{\epsilon(\alpha)} \rho, \nu^{\alpha+m} \rho\right]\right) \times \delta\left(\left[\nu^{1-\epsilon(\alpha)} \rho, \nu^{\alpha+n} \rho\right]\right) \otimes \sigma$ is $2(1-\epsilon(\alpha))$. Let $\pi^{\prime}$ be an irreducible subquotient of $\delta\left(\left[\nu^{-\alpha-n} \rho, \nu^{\alpha+m} \rho\right]\right) \rtimes \sigma$ which satisfies $\delta\left(\left[\nu^{-\alpha-n} \rho, \nu^{\alpha+m} \rho\right]\right) \otimes \sigma \leq s_{G L}\left(\pi^{\prime}\right)$ and $\delta\left(\left[\nu^{\epsilon(\alpha)} \rho, \nu^{\alpha+m} \rho\right]\right) \times \delta\left(\left[\nu^{1-\epsilon(\alpha)} \rho\right.\right.$, $\left.\left.\nu^{\alpha+n} \rho\right]\right) \otimes \sigma \not \leq s_{G L}\left(\pi^{\prime}\right)$. Then the multiplicity of $\pi^{\prime}$ in $\delta\left(\left[\nu^{-\alpha-n} \rho, \nu^{\alpha+m} \rho\right]\right) \rtimes \sigma$ is one and $\pi^{\prime} \cong \delta\left(\left[\nu^{-\alpha-n} \rho, \nu^{\alpha+m} \rho\right]_{-}, \sigma\right)$.

4.3. Lemma. For $-\alpha-n \leq j<0, j \in \alpha+\mathbb{Z}, \delta\left(\left[\nu^{j} \rho, \nu^{\alpha+m} \rho\right]\right) \times \delta\left(\left[\nu^{-j+1} \rho, \nu^{\alpha+n} \rho\right]\right)$ $\otimes \sigma$ is irreducible and its multiplicity in $\left.s_{G L}\left(\delta\left(\left[\nu^{-\alpha-n} \rho, \nu^{\alpha+m} \rho\right]\right) \rtimes \sigma\right)\right)$ is two.

Proof. Since $j<-j+1$ when $j<0$, we have irreducibility. Suppose that $\delta\left(\left[\nu^{j} \rho, \nu^{\alpha+m} \rho\right]\right) \times \delta\left(\left[\nu^{-j+1} \rho, \nu^{\alpha+n} \rho\right]\right) \otimes \sigma$ is a subquotient of the $i$-th term in the sum (3-2) for fixed $j$ as in the lemma. Considering $\nu^{j-1} \rho$, which is not in the $G L$ support of $\delta\left(\left[\nu^{j} \rho, \nu^{\alpha+m} \rho\right]\right) \times \delta\left(\left[\nu^{-j+1} \rho, \nu^{\alpha+n} \rho\right]\right) \otimes \sigma$, we get that $i \geq j$ and $-i+1 \geq$ $j$. Since $\nu^{j} \rho$ is in the $G L$-support of $\delta\left(\left[\nu^{j} \rho, \nu^{\alpha+m} \rho\right]\right) \times \delta\left(\left[\nu^{-j+1} \rho, \nu^{\alpha+n} \rho\right]\right) \otimes \sigma$, we get $i=j$ or $-i+1=j$ (i.e., $i=-j+1$ ). Now the $j$-th term is just $\delta\left(\left[\nu^{j} \rho, \nu^{\alpha+m} \rho\right]\right) \times \delta\left(\left[\nu^{-j+1} \rho, \nu^{\alpha+n} \rho\right]\right) \otimes \sigma$. Further, $(-j+1)$-th term is $\delta\left(\left[\nu^{-j+1} \rho\right.\right.$, $\left.\left.\nu^{\alpha+m} \rho\right]\right) \times \delta\left(\left[\nu^{j} \rho, \nu^{\alpha+n} \rho\right]\right) \otimes \sigma$. Since $j<-j+1, \alpha+n<\alpha+m$ and $-j \leq$ $\alpha+n$, the segments in the last representation are linked, and linking them we get $\delta\left(\left[\nu^{j} \rho, \nu^{\alpha+m} \rho\right]\right) \times \delta\left(\left[\nu^{-j+1} \rho, \nu^{\alpha+n} \rho\right]\right) \otimes \sigma$. Therefore, the multiplicity in the $(-j+1)$-th term is also one. Since $j \neq-j+1$ for $j$ as in the lemma, we get that the multiplicity is two.

The proof of the following lemma proceeds similarly to the proof of Lemma 2.1.

4.4. Lemma. Let $\pi$ be an irreducible subrepresentation of $\delta\left(\left[\nu^{-\alpha-n} \rho, \nu^{\alpha+m} \rho\right]\right) \rtimes \sigma$ satisfying $s_{G L}(\pi) \leq k \sum_{j=-\alpha-n}^{\alpha} \delta\left(\left[\nu^{j} \rho, \nu^{\alpha+m} \rho\right]\right) \times \delta\left(\left[\nu^{-j+1} \rho, \nu^{\alpha+n} \rho\right]\right) \otimes \sigma$, for some $k \in \mathbb{Z}, k>0$. Then $s_{G L}(\pi) \geq \sum_{j=-\alpha-n}^{-\alpha} \delta\left(\left[\nu^{j} \rho, \nu^{\alpha+m} \rho\right]\right) \times \delta\left(\left[\nu^{-j+1} \rho, \nu^{\alpha+n} \rho\right]\right) \otimes \sigma$. 
Proof. First, we shall prove by induction

$$
\begin{array}{r}
\pi \hookrightarrow\left(\nu^{\alpha+m} \rho \times \nu^{\alpha+m-1} \rho \times \cdots \times \nu^{\epsilon(\alpha)} \rho\right) \times\left(\nu^{\alpha+n} \rho \times \nu^{\alpha+n-1} \rho \times \cdots \times \nu^{-i+1} \rho\right) \\
\times\left(\nu^{\epsilon(\alpha)-1} \rho \times \nu^{\epsilon(\alpha)-2} \rho \times \cdots \times \nu^{i} \rho\right) \rtimes \sigma
\end{array}
$$

for $i=-\alpha-n,-\alpha-n+1, \ldots,-\alpha$. Since we know that $\pi \hookrightarrow \delta\left(\left[\nu^{-\alpha-n} \rho, \nu^{\alpha+m} \rho\right]\right)$ $\rtimes \sigma \hookrightarrow \nu^{\alpha+m} \rho \times \nu^{\alpha+m-1} \rho \times \cdots \times \nu^{-\alpha-n+1} \rho \times \nu^{-\alpha-n} \rho \rtimes \sigma$, the claim holds for $i=-\alpha-n$. Let $-\alpha-n \leq i<-\alpha$, and suppose that the claim holds for this $i$. It is worth noting that the isomorphism $\nu^{i} \rho \rtimes \sigma \cong \nu^{-i} \rho \rtimes \sigma$ follows from the irreducibility of $\nu^{i} \rho \rtimes \sigma$ (recall $-i>\alpha$ ). Further, for $j=i+1, i+2, \ldots, \epsilon(\alpha)-1$, we have $-i>\alpha>\epsilon(\alpha)-1 \geq j$, which implies $-i \geq j+2$. Therefore, $\nu^{-i} \rho \times \nu^{j} \rho \cong$ $\nu^{j} \rho \times \nu^{-i} \rho$. These two facts imply the claim for $i+1$ (see (2-6) for a proof of a similar implication). This ends the proof of the above embedding. Now Frobenius reciprocity implies that

$$
\begin{aligned}
\tau_{i}= & \left(\nu^{\alpha+m} \rho \otimes \nu^{\alpha+m-1} \rho \otimes \cdots \otimes \nu^{\epsilon(\alpha)} \rho\right) \otimes\left(\nu^{\alpha+n} \rho \otimes \nu^{\alpha+n-1} \rho \otimes \cdots \otimes \nu^{-i+1} \rho\right) \\
& \otimes\left(\nu^{\epsilon(\alpha)-1} \rho \otimes \nu^{\epsilon(\alpha)-2} \rho \otimes \cdots \otimes \nu^{i} \rho\right) \otimes \sigma, \quad i=\alpha+1, \alpha+2, \ldots, \alpha+n+1,
\end{aligned}
$$

is a subquotient of the corresponding Jacquet module of $\pi$. The transitivity of Jacquet functors implies that $\tau_{i}$ is in a corresponding Jacquet module of at least one irreducible subquotient of $s_{G L}(\pi)$. Thus, it must be in the corresponding Jacquet module of at least one irreducible subquotient of the upper bound of $s_{G L}(\pi)$ given in the lemma. Since for $-\alpha+1 \leq j \leq \alpha, \nu^{i} \rho$ is not in the $G L$-support of the corresponding term in the upper bound ( $i$ is as above), we see that $\tau_{i}$ can be a subquotient only of a term corresponding to $-\alpha-n \leq j \leq-\alpha$. Note that the terms corresponding to these indexes are irreducible representations. Since for $j=i, \nu^{i} \rho$ is and $\nu^{i-1} \rho$ is not in the $G L$-support of the corresponding term, and this is the only term satisfying this condition, we see that $\tau_{i}$ must be a subquotient of the $i$-th term. Since this is the only irreducible subquotient in the upper bound which has $\tau_{i}$ in the corresponding Jacquet module, this implies that the $i$-th term in the upper bound must be a subquotient of $s_{G L}(\pi)$. Since the terms in the upper bound corresponding to different $-\alpha+1 \leq i \leq \alpha$ are inequivalent, the claim of the lemma follows directly.

4.5. Theorem. Suppose that $\rho$ and $\sigma$ are irreducible unitarizable cuspidal representations of $G L(p, F)$ and $S_{q}$ respectively, and $\alpha \in(1 / 2) \mathbb{Z}, \alpha>0$, is such that $\nu^{\alpha} \rho \rtimes \sigma$ reduces and $\nu^{\beta} \rho \rtimes \sigma$ is irreducible for $\beta \in(\alpha+\mathbb{Z}) \backslash\{ \pm \alpha\}$. Fix $n, m \in \mathbb{Z}$ satisfying $0 \leq n<m$. Then

$$
\begin{aligned}
s_{G L}( & \left.\delta\left(\left[\nu^{-\alpha-n} \rho, \nu^{\alpha+m} \rho\right]_{-}, \sigma\right)\right) \\
& =\sum_{i=-\alpha-n}^{-\alpha} \delta\left(\left[\nu^{i} \rho, \nu^{\alpha+m} \rho\right]\right) \times \delta\left(\left[\nu^{-i+1} \rho, \nu^{\alpha+n} \rho\right]\right) \otimes \sigma,
\end{aligned}
$$

and $\delta\left(\left[\nu^{-\alpha-n} \rho, \nu^{\alpha+m} \rho\right]_{-}, \sigma\right)$ is square integrable. (Note that (4-12) holds also if $n=m$.)

Proof. Observe $\delta\left(\left[\nu^{-\alpha-n} \rho, \nu^{\alpha+m} \rho\right]\right) \rtimes \sigma \leq \delta\left(\left[\nu^{-\alpha+1} \rho, \nu^{\alpha+m} \rho\right]\right) \times \delta\left(\left[\nu^{\alpha} \rho, \nu^{\alpha+n} \rho\right]\right) \rtimes \sigma$. Proposition 3.2 implies

$$
\delta\left(\left[\nu^{-\alpha-n} \rho, \nu^{\alpha+m} \rho\right]_{ \pm}, \sigma\right) \leq \delta\left(\left[\nu^{-\alpha+1} \rho, \nu^{\alpha+m} \rho\right]\right) \times \delta\left(\left[\nu^{\alpha} \rho, \nu^{\alpha+n} \rho\right]\right) \rtimes \sigma,
$$


Write

$$
\begin{aligned}
& \text { s.s. }\left(s_{G L}\left(\delta\left(\left[\nu^{-\alpha+1} \rho, \nu^{\alpha+m} \rho\right]\right) \times \delta\left(\left[\nu^{\alpha} \rho, \nu^{\alpha+n} \rho\right], \sigma\right)\right)\right) \\
& \quad=\delta\left(\left[\nu^{\alpha} \rho, \nu^{\alpha+n} \rho\right]\right) \times \sum_{i=-\alpha+1}^{\alpha+m+1} \delta\left(\left[\nu^{i} \rho, \nu^{\alpha+m} \rho\right]\right) \times \delta\left(\left[\nu^{-i+1} \rho, \nu^{\alpha-1} \rho\right]\right) \otimes \sigma .
\end{aligned}
$$

Note that $\delta\left(\left[\rho, \nu^{\alpha+m} \rho\right]\right) \times \delta\left(\left[\nu \rho, \nu^{\alpha+n} \rho\right]\right) \otimes \sigma\left(\operatorname{resp} . \quad \delta\left(\left[\nu^{1 / 2} \rho, \nu^{\alpha+m} \rho\right]\right) \times \delta\left(\left[\nu^{1 / 2} \rho\right.\right.\right.$, $\left.\left.\left.\nu^{\alpha+n} \rho\right]\right) \otimes \sigma\right)$ is a subquotient of (4-14) if $\alpha \in \mathbb{Z}$ (resp. $\alpha \in(1 / 2+\mathbb{Z})$ ). This follows considering the term corresponding to $i=0$ (resp. $i=1 / 2$ ). Now Lemma 4.1 implies

$$
\delta\left(\left[\nu^{-\alpha-n} \rho, \nu^{\alpha+m} \rho\right], \sigma\right) \leq \delta\left(\left[\nu^{-\alpha+1} \rho, \nu^{\alpha+m} \rho\right]\right) \rtimes \delta\left(\left[\nu^{\alpha} \rho, \nu^{\alpha+n} \rho\right], \sigma\right) .
$$

One directly checks that the multiplicity of $\delta\left(\left[\nu^{-\alpha-n} \rho, \nu^{\alpha+m} \rho\right]\right) \otimes \sigma$ in (4-14) is one (it must be a subquotient of the $(\alpha+n+1)$-th term in the sum). This fact, (4-13) and Proposition 4.2 imply

$$
\begin{aligned}
& \delta\left(\left[\nu^{-\alpha-n} \rho, \nu^{\alpha+m} \rho\right]_{-}, \sigma\right) \\
& \quad \leq \delta\left(\left[\nu^{-\alpha+1} \rho, \nu^{\alpha+m} \rho\right]\right) \rtimes\left(\delta\left(\left[\nu^{\alpha} \rho, \nu^{\alpha+n} \rho\right]\right) \rtimes \sigma-\delta\left(\left[\nu^{\alpha} \rho, \nu^{\alpha+n} \rho\right], \sigma\right)\right) .
\end{aligned}
$$

This inequality and Proposition 3.2 imply

$$
\begin{aligned}
& s_{G L}(\left.\delta\left(\left[\nu^{-\alpha-n} \rho, \nu^{\alpha+m} \rho\right]_{-}, \sigma\right)\right) \\
& \leq \sum_{i=-\alpha+1}^{\alpha+m+1} \delta\left(\left[\nu^{i} \rho, \nu^{\alpha+m} \rho\right]\right) \times \delta\left(\left[\nu^{-i+1} \rho, \nu^{\alpha-1} \rho\right]\right) \\
& \quad \times \sum_{j=\alpha+1}^{\alpha+n+1} \delta\left(\left[\nu^{j} \rho, \nu^{\alpha+n} \rho\right]\right) \times \delta\left(\left[\nu^{-j+1} \rho, \nu^{-\alpha} \rho\right]\right) \otimes \sigma, \\
& s_{G L}\left(\delta\left(\left[\nu^{-\alpha-n} \rho, \nu^{\alpha+m} \rho\right]_{-}, \sigma\right)\right) \\
& \leq \sum_{i=-\alpha-n}^{\alpha+m+1} \delta\left(\left[\nu^{i} \rho, \nu^{\alpha+m} \rho\right]\right) \times \delta\left(\left[\nu^{-i+1} \rho, \nu^{\alpha+n} \rho\right]\right) \otimes \sigma .
\end{aligned}
$$

Suppose that $\pi$ is an irreducible subquotient of a term on the right-hand side of (4-17) which corresponds to indexes $i$ and $j$, and which is also a subquotient of a term on the right-hand side of (4-18) corresponding to $i^{\prime}$. Note that $\nu^{-\alpha} \rho$ is in the $G L$-support of all the (irreducible subquotients of) terms in the second sum on the right-hand side of (4-17) (consider $\delta\left(\left[\nu^{-j+1} \rho, \nu^{-\alpha} \rho\right]\right)$ ). Since $\nu^{-\alpha} \rho$ can be at most once in the $G L$-support of a subquotient of the right-hand side of (4-18), we get $-\alpha<-i+1$, and further $i<\alpha+1$. Since $\nu^{-\alpha} \rho$ is in the $G L$-support of $\pi$ (as we already observed), (4-18) implies that $\nu^{-\alpha+1} \rho$ is also in the support of $\pi$. This implies $i \leq-\alpha+1$ or $-i+1 \leq-\alpha+1$ (since $-\alpha+1<\alpha+1$ ), i.e., $i=-\alpha+1$ or $\alpha \leq i$. Thus, $i=-\alpha+1$ or $i=\alpha$ (since we know $i<-\alpha+1$ already). The above discussion and (4-17) imply

$$
\begin{aligned}
& s_{G L}\left(\delta\left(\left[\nu^{-\alpha-n} \rho, \nu^{\alpha+m} \rho\right]_{-}, \sigma\right)\right) \\
& \leq\left(\delta\left(\left[\nu^{-\alpha+1} \rho, \nu^{\alpha+m} \rho\right]\right)+\delta\left(\left[\nu^{\alpha} \rho, \nu^{\alpha+m} \rho\right]\right) \times \delta\left(\left[\nu^{-\alpha+1} \rho, \nu^{\alpha-1} \rho\right]\right)\right) \\
& \times \sum_{j=\alpha+1}^{\alpha+n+1} \delta\left(\left[\nu^{j} \rho, \nu^{\alpha+n} \rho\right]\right) \times \delta\left(\left[\nu^{-j+1} \rho, \nu^{-\alpha} \rho\right]\right) \otimes \sigma .
\end{aligned}
$$


Further, (4-18) and the fact that $\nu^{-\alpha} \rho$ is always in the $G L$-support of $\pi$ imply

$$
\begin{aligned}
s_{G L}\left(\delta\left(\left[\nu^{-\alpha-n} \rho, \nu^{\alpha+m} \rho\right]_{-}, \sigma\right)\right) \\
\leq \sum_{i=-\alpha-n}^{-\alpha} \delta\left(\left[\nu^{i} \rho, \nu^{\alpha+m} \rho\right]\right) \times \delta\left(\left[\nu^{-i+1} \rho, \nu^{\alpha+n} \rho\right]\right) \otimes \sigma \\
\quad+\sum_{i=\alpha+1}^{\alpha+n+1} \delta\left(\left[\nu^{i} \rho, \nu^{\alpha+m} \rho\right]\right) \times \delta\left(\left[\nu^{-i+1} \rho, \nu^{\alpha+n} \rho\right]\right) \otimes \sigma .
\end{aligned}
$$

Note that terms in the third row of (4-20) are multiplicity one representations of length two. From (4-19) we see that if $\pi=L\left(\delta\left(\Delta_{1}\right), \ldots, \delta\left(\Delta_{k}\right)\right) \otimes \sigma$ and if some $\Delta_{j}$ ends with $\nu^{\alpha+m} \rho$, then $\Delta_{j}$ must also contain $\nu^{\alpha} \rho$. This fact and (4-20) imply

$$
\begin{aligned}
s_{G L}( & \left.\delta\left(\left[\nu^{-\alpha-n} \rho, \nu^{\alpha+m} \rho\right]_{-}, \sigma\right)\right) \\
\leq & \sum_{i=-\alpha-n}^{-\alpha} \delta\left(\left[\nu^{i} \rho, \nu^{\alpha+m} \rho\right]\right) \times \delta\left(\left[\nu^{-i+1} \rho, \nu^{\alpha+n} \rho\right]\right) \otimes \sigma \\
& \quad+\sum_{i=\alpha+1}^{\alpha+n+1} \delta\left(\left[\nu^{-i+1} \rho, \nu^{\alpha+m} \rho\right]\right) \times \delta\left(\left[\nu^{i} \rho, \nu^{\alpha+n} \rho\right]\right) \otimes \sigma \\
\quad= & 2 \sum_{i=-\alpha-n}^{-\alpha} \delta\left(\left[\nu^{i} \rho, \nu^{\alpha+m} \rho\right]\right) \times \delta\left(\left[\nu^{-i+1} \rho, \nu^{\alpha+n} \rho\right]\right) \otimes \sigma .
\end{aligned}
$$

Now the square integrability of $\delta\left(\left[\nu^{-\alpha-n} \rho, \nu^{\alpha+m} \rho\right]_{-}, \sigma\right)$ follows from the above estimate, using the Casselman's square integrability criterion (Theorem 4.4.6 of [C1]; see also the sixth section of [T2]).

Further, (4-21) and Lemma 4.4 imply that the inequality $\geq$ holds in (4-12). It is enough to show that the inequality $\leq$ holds in (4-12). There are two ways to prove that. The shorter way is to conclude it from (4-23), which we shall prove without using (4-12), and from Lemma 4.3. The other way is to get it from (4-21) and the following lemma.

4.6. Lemma. (i) $\delta\left(\left[\nu^{-\alpha-n} \rho, \nu^{\alpha+m} \rho\right]_{-}, \sigma\right) \leq \delta\left(\left[\nu^{\alpha+n+1} \rho, \nu^{\alpha+m} \rho\right]\right) \times \delta\left(\left[\nu^{-\alpha-n} \rho\right.\right.$, $\left.\left.\nu^{\alpha+n} \rho\right]_{-}, \sigma\right)$.

(ii) $s_{G L}\left(\delta\left(\left[\nu^{\alpha+n+1} \rho, \nu^{\alpha+m} \rho\right]\right) \times \delta\left(\left[\nu^{-\alpha-n} \rho, \nu^{\alpha+n} \rho\right]_{-}, \sigma\right)\right)$ is a multiplicity one representation.

Proof. First, we obtain directly $\delta\left(\left[\nu^{-\alpha-n} \rho, \nu^{\alpha+m} \rho\right]\right) \otimes \sigma \leq s_{G L}\left(\delta\left(\left[\nu^{\alpha+n+1} \rho, \nu^{\alpha+m} \rho\right]\right)\right.$ $\left.\times \delta\left(\left[\nu^{-\alpha-n} \rho, \nu^{\alpha+n} \rho\right]_{-}, \sigma\right)\right)$. Further, we note that $\delta\left(\left[\rho, \nu^{\alpha+m} \rho\right]\right) \times \delta\left(\left[\nu \rho, \nu^{\alpha+n} \rho\right]\right)$ $\otimes \sigma\left(\right.$ resp. $\left.\quad \delta\left(\left[\nu^{1 / 2} \rho, \nu^{\alpha+m} \rho\right]\right) \times \delta\left(\left[\nu^{1 / 2} \rho, \nu^{\alpha+n} \rho\right]\right) \otimes \sigma\right)$ is not a subquotient of $s_{G L}\left(\delta\left(\left[\nu^{\alpha+n+1} \rho, \nu^{\alpha+m} \rho\right]\right) \times \delta\left(\left[\nu^{-\alpha-n} \rho, \nu^{\alpha+n} \rho\right]_{-}, \sigma\right)\right)$ if $\alpha \in \mathbb{Z}($ resp. $\alpha \in(1 / 2+\mathbb{Z}))$. These facts follow from (2-20) and (1-4), and together with the fact that the multiplicity of $\delta\left(\left[\nu^{-\alpha-n} \rho, \nu^{\alpha+m} \rho\right]\right) \otimes \sigma$ in $s_{G L}\left(\delta\left(\left[\nu^{\alpha+n+1} \rho, \nu^{\alpha+m} \rho\right]\right) \times \delta\left(\left[\nu^{-\alpha-n} \rho, \nu^{\alpha+n} \rho\right]\right)\right.$ $\rtimes \sigma$ ) is two (by (i) of Lemma 4.1), imply (i). Now write

$$
\begin{aligned}
& \text { s.s. }\left(s_{G L}\left(\delta\left(\left[\nu^{\alpha+n+1} \rho, \nu^{\alpha+m} \rho\right]\right) \times \delta\left(\left[\nu^{-\alpha-n} \rho, \nu^{\alpha+n} \rho\right]_{-}, \sigma\right)\right)\right) \\
&=\sum_{i=\alpha+n+1}^{\alpha+m+1} \delta\left(\left[\nu^{i} \rho, \nu^{\alpha+m} \rho\right]\right) \times \delta\left(\left[\nu^{-i+1} \rho, \nu^{-\alpha-n-1} \rho\right]\right) \\
& \quad \times \sum_{j=-\alpha-n}^{-\alpha} \delta\left(\left[\nu^{j} \rho, \nu^{\alpha+n} \rho\right]\right) \times \delta\left(\left[\nu^{-j+1} \rho, \nu^{\alpha+n} \rho\right]\right) \otimes \sigma .
\end{aligned}
$$


Note that all the terms in the above double sum have different $G L$-supports. Because of this, to prove (ii), it is enough to prove that each of the terms is a multiplicity one representation. If $i=\alpha+n+1$ or $\alpha+m+1$, then Lemma 7.1 implies the multiplicity one of the corresponding term (note that Lemma 7.1 is in the appendix, which is independent of the rest of the text). Suppose that $\alpha+n+2 \leq i \leq \alpha+m$ and $-\alpha-n \leq j \leq-\alpha$. Then the segment $\left[\nu^{i} \rho, \nu^{\alpha+m} \rho\right]$ is not linked with any other segment which shows up in the term corresponding to $i$ and $j$. This and Lemma 7.1 imply the multiplicity one.

4.7. Theorem. Let $\rho, \sigma, \alpha, n$ and $m$ be as in Theorem 4.5. Then

$$
\begin{aligned}
& s_{G L}(\left.\delta\left(\left[\nu^{-\alpha-n} \rho, \nu^{\alpha+m} \rho\right], \sigma\right)\right) \\
& \leq \sum_{i=-\alpha-n}^{\alpha} \delta\left(\left[\nu^{i} \rho, \nu^{\alpha+m} \rho\right]\right) \times \delta\left(\left[\nu^{-i+1} \rho, \nu^{\alpha+n} \rho\right]\right) \otimes \sigma, \\
& 2(1-\epsilon(\alpha)) \delta\left(\left[\nu^{\epsilon(\alpha)} \rho, \nu^{\alpha+m} \rho\right]\right) \times \delta\left(\left[\nu^{1-\epsilon(\alpha)} \rho, \nu^{\alpha+n} \rho\right]\right) \otimes \sigma \\
& \quad+\sum_{i=-\alpha-n}^{-\alpha} \delta\left(\left[\nu^{i} \rho, \nu^{\alpha+m} \rho\right]\right) \times \delta\left(\left[\nu^{-i+1} \rho, \nu^{\alpha+n} \rho\right]\right) \otimes \sigma \\
& \leq s_{G L}\left(\delta\left(\left[\nu^{-\alpha-n} \rho, \nu^{\alpha+m} \rho\right], \sigma\right)\right),
\end{aligned}
$$

and $\delta\left(\left[\nu^{-\alpha-n} \rho, \nu^{\alpha+m} \rho\right], \sigma\right)$ is square integrable. (The above estimates also hold if $n=m$.)

Proof. Denote $\pi=\delta\left(\left[\nu^{-\alpha-n} \rho, \nu^{\alpha+m} \rho\right], \sigma\right)$.

Observe that the representation $\delta\left(\left[\nu^{\epsilon(\alpha)} \rho, \nu^{\alpha+m} \rho\right]\right) \times \delta\left(\left[\nu^{1-\epsilon(\alpha)} \rho, \nu^{\alpha+n} \rho\right]\right) \otimes \sigma$ is a subquotient $s_{G L}\left(\delta\left(\left[\nu^{-\alpha+1} \rho, \nu^{\alpha+n} \rho\right]\right) \rtimes \delta\left(\left[\nu^{\alpha} \rho, \nu^{\alpha+m} \rho\right], \sigma\right)\right)$ (in the sum of (4-14) consider the term in the sum corresponding to $i=\epsilon(\alpha)$; note $-\alpha+1 \leq \epsilon(\alpha) \leq \alpha+$ $m+1)$. Now Proposition 4.2 implies $\pi \leq \delta\left(\left[\nu^{-\alpha+1} \rho, \nu^{\alpha+n} \rho\right]\right) \rtimes \delta\left(\left[\nu^{\alpha} \rho, \nu^{\alpha+m} \rho\right], \sigma\right)$. This fact and $\pi \leq \delta\left(\left[\nu^{-\alpha-n} \rho, \nu^{\alpha+m} \rho\right]\right) \rtimes \sigma$ imply

$$
\begin{aligned}
s_{G L}(\pi) \leq & \delta\left(\left[\nu^{\alpha} \rho, \nu^{\alpha+m} \rho\right]\right) \\
& \times \sum_{i=-\alpha+1}^{\alpha+n+1} \delta\left(\left[\nu^{i} \rho, \nu^{\alpha+n} \rho\right]\right) \times \delta\left(\left[\nu^{-i+1} \rho, \nu^{\alpha-1} \rho\right]\right) \otimes \sigma, \\
s_{G L}(\pi) \leq & \sum_{i=-\alpha-n}^{\alpha+m+1} \delta\left(\left[\nu^{i} \rho, \nu^{\alpha+m} \rho\right]\right) \times \delta\left(\left[\nu^{-i+1} \rho, \nu^{\alpha+n} \rho\right]\right) \otimes \sigma .
\end{aligned}
$$

Now (4-24) implies that $\nu^{-\alpha-n-1} \rho$ cannot be in the $G L$-support of irreducible subquotients of $s_{G L}(\pi)$. Therefore, we get from (4-25) the following estimate

$$
\begin{aligned}
& s_{G L}(\left.\delta\left(\left[\nu^{-\alpha-n} \rho, \nu^{\alpha+m} \rho\right], \sigma\right)\right) \\
& \quad \leq \sum_{i=-\alpha-n}^{\alpha+n+1} \delta\left(\left[\nu^{i} \rho, \nu^{\alpha+m} \rho\right]\right) \times \delta\left(\left[\nu^{-i+1} \rho, \nu^{\alpha+n} \rho\right]\right) \otimes \sigma .
\end{aligned}
$$

A term in the above sum is either irreducible, or a multiplicity one representation of length two. Let $\tau=L\left(\delta\left(\Delta_{1}\right), \ldots, \delta\left(\Delta_{k}\right)\right) \otimes \sigma$ be an irreducible subquotient of 
the Jacquet module $s_{G L}(\pi)$. Now (4-24) implies that if some $\Delta_{j}$ ends with $\nu^{\alpha+m} \rho$, then $\nu^{\alpha} \rho$ must also be in this $\Delta_{j}$. Therefore, (4-26) now implies

$$
\begin{aligned}
s_{G L}(\pi) \leq & \sum_{i=-\alpha-n}^{\alpha} \delta\left(\left[\nu^{i} \rho, \nu^{\alpha+m} \rho\right]\right) \times \delta\left(\left[\nu^{-i+1} \rho, \nu^{\alpha+n} \rho\right]\right) \otimes \sigma \\
& +\sum_{i=\alpha+1}^{\alpha+n+1} \delta\left(\left[\nu^{-i+1} \rho, \nu^{\alpha+m} \rho\right]\right) \times \delta\left(\left[\nu^{i} \rho, \nu^{\alpha+n} \rho\right]\right) \otimes \sigma \\
= & \sum_{i=-\alpha-n}^{\alpha} \delta\left(\left[\nu^{i} \rho, \nu^{\alpha+m} \rho\right]\right) \times \delta\left(\left[\nu^{-i+1} \rho, \nu^{\alpha+n} \rho\right]\right) \otimes \sigma \\
+ & \sum_{i=-\alpha-n}^{-\alpha} \delta\left(\left[\nu^{i} \rho, \nu^{\alpha+m} \rho\right]\right) \times \delta\left(\left[\nu^{-i+1} \rho, \nu^{\alpha+n} \rho\right]\right) \otimes \sigma . \\
& =\sum_{i=-\alpha+1}^{\alpha} \delta\left(\left[\nu^{i} \rho, \nu^{\alpha+m} \rho\right]\right) \times \delta\left(\left[\nu^{-i+1} \rho, \nu^{\alpha+n} \rho\right]\right) \otimes \sigma \\
& +2 \sum_{i=-\alpha-n}^{-\alpha} \delta\left(\left[\nu^{i} \rho, \nu^{\alpha+m} \rho\right]\right) \times \delta\left(\left[\nu^{-i+1} \rho, \nu^{\alpha+n} \rho\right]\right) \otimes \sigma .
\end{aligned}
$$

This estimate and Lemma 4.4 imply (4-23) (note that we have not used (4-12) in the proof of the estimate, and also not in the proof of (4-23)). Further, the square integrability of $\pi$ follows from the estimate, using the Casselman's square integrability criterion (Theorem 4.4.6 of [C1]). At the end, Lemma 4.3, (4-12) and the exactness of the Jacquet functor imply (4-22).

4.8. Remarks. (i) Note that for $\alpha=1 / 2$, the estimates in the above theorem imply

$$
\begin{aligned}
\text { s.s. }( & \left.\delta\left(\left[\nu^{-1 / 2-n} \rho, \nu^{1 / 2+m} \rho\right], \sigma\right)\right) \\
& =\sum_{i=-1 / 2-n}^{1 / 2} \delta\left(\left[\nu^{i} \rho, \nu^{1 / 2+m} \rho\right]\right) \times \delta\left(\left[\nu^{-i+1} \rho, \nu^{1 / 2+n} \rho\right]\right) \otimes \sigma .
\end{aligned}
$$

(ii) Let us consider the groups $S O(2 q+1, F)$ and let $\chi$ be a character of $G L(1, F)=$ $F^{\times}$such that $\chi^{2}$ is the trivial character. The one-dimensional representation of $S O(1, F)$ will be denoted by 1 . Let integers $n$ and $m$ satisfy $0 \leq n<m$. Theorem 4.5 and (i) imply that the lengths of the Jacquet modules of representations $\delta\left(\left[\nu^{-1 / 2-n} \chi, \nu^{1 / 2+m} \chi\right], 1\right)$ and $\delta\left(\left[\nu^{-1 / 2-n} \chi, \nu^{1 / 2+m} \chi\right]_{-}, 1\right)$ with respect to a minimal parabolic subgroup are given by $\sum_{i=0}^{n+1}\left(\begin{array}{c}n+m+2 \\ i\end{array}\right)$ and $\sum_{i=0}^{n}\left(\begin{array}{c}n+m+2 \\ i\end{array}\right)$, respectively. Suppose that $\chi$ is unramified. Then Lemma 3 of [Ro] (or Propositions 2.4 and 2.5 of [C2]) implies that the dimensions of the spaces of the Iwahori fixed vectors in the representations $\delta\left(\left[\nu^{-1 / 2-n} \chi, \nu^{1 / 2+m} \chi\right], 1\right)$ and $\delta\left(\left[\nu^{-1 / 2-n} \chi, \nu^{1 / 2+m} \chi\right]_{-}, 1\right)$ are given respectively again by

$$
\sum_{i=0}^{n+1}\left(\begin{array}{c}
n+m+2 \\
i
\end{array}\right) \text { and } \sum_{i=0}^{n}\left(\begin{array}{c}
n+m+2 \\
i
\end{array}\right) .
$$

\section{Square integrability, the Case $\alpha=0$}

In this section $\rho$ and $\sigma$ are as in the previous sections (irreducible unitarizable cuspidal representations of $G L(p, F)$ and $S_{q}$, respectively). Further, $n$ and $m$ are 
integers satisfying $0 \leq n<m$. We shall assume in this section that $\rho \rtimes \sigma$ reduces (then $\rho \cong \tilde{\rho}$ ) and that $\nu^{\alpha} \rho \rtimes \sigma$ is irreducible for $\alpha \in \mathbb{Z} \backslash\{0\}$. Write $\rho \rtimes \sigma=\tau_{1} \oplus \tau_{2}$ as a sum of irreducible representations (then $\tau_{1} ¥ \tau_{2}$ ).

5.1. Lemma. The representation $\delta\left([\rho, \nu \rho]_{\tau_{i}}, \sigma\right)$ is square integrable. It is the only square integrable subquotient of $\nu \rho \rtimes \tau_{i}$ and we have

$$
\mu^{*}\left(\delta\left([\rho, \nu \rho]_{\tau_{i}}, \sigma\right)\right)=1 \otimes \delta\left([\rho, \nu \rho]_{\tau_{i}}, \sigma\right)+\nu \rho \otimes \tau_{i}+\delta([\rho, \nu \rho]) \otimes \sigma .
$$

Proof. We have an epimorphism $\nu \rho \rtimes \tau_{i} \rightarrow L\left(\nu \rho, \tau_{i}\right)$. Replacing $\tau_{i}$ by $\tilde{\tau}_{i}$ in the endomorphisms and passing to contragredients, one gets monomorphisms $L\left(\nu \rho, \tilde{\tau}_{i}\right)^{\sim} \hookrightarrow$ $\nu^{-1} \rho \rtimes \tau_{i}$. Since $L\left(\nu \rho, \tilde{\tau}_{i}\right)^{\sim} \cong L\left(\nu \rho, \tau_{i}\right)$, Frobenius reciprocity implies that there exist epimorphisms

$$
s_{(p)}\left(L\left(\nu \rho, \tau_{i}\right)\right) \rightarrow \nu^{-1} \rho \otimes \tau_{i} .
$$

Further, we have an epimorphism $\delta([\rho, \nu \rho]) \rtimes \sigma \rightarrow L(\delta([\rho, \nu \rho]), \sigma)$. A similar argument then gives us an epimorphism

$$
s_{G L}(L(\delta([\rho, \nu \rho]), \sigma)) \rightarrow \delta\left(\left[\nu^{-1} \rho, \rho\right]\right) \otimes \sigma .
$$

Now write

$$
\begin{aligned}
\mu^{*}\left(\nu \rho \rtimes \tau_{i}\right)= & 1 \otimes \nu \rho \rtimes \tau_{i}+\left[\nu \rho \otimes \tau_{i}+\nu^{-1} \rho \otimes \tau_{i}+\rho \otimes \nu \rho \rtimes \sigma\right] \\
& +\left[\nu \rho \times \rho \otimes \sigma+\nu^{-1} \rho \times \rho \otimes \sigma\right] .
\end{aligned}
$$

From the above formula, we see that $\nu \rho \times \rho \rtimes \sigma$ is a representation of length $\leq 6$ because

$$
\nu \rho \times \rho \rtimes \sigma=\nu \rho \rtimes \tau_{1} \oplus \nu \rho \rtimes \tau_{2} .
$$

Also, from (5-1) and (5-3), one gets that each $L\left(\nu \rho, \tau_{i}\right)$ has multiplicity one in $\nu \rho \times \rho \rtimes \sigma$. Further, there is an exact sequence of representations

$$
0 \rightarrow \delta([\rho, \nu \rho]) \rtimes \sigma \stackrel{\alpha}{\longrightarrow} \nu \rho \times \rho \rtimes \sigma \stackrel{\beta}{\longrightarrow} L(\nu \rho, \rho) \rtimes \sigma \longrightarrow 0 .
$$

We have

$$
\begin{aligned}
& \mu^{*}(\delta([\rho, \nu \rho]) \rtimes \sigma)=1 \otimes \delta([\rho, \nu \rho]) \rtimes \sigma+\left[\nu \rho \otimes \tau_{1}+\nu \rho \otimes \tau_{2}+\rho \otimes \nu \rho \rtimes \sigma\right] \\
&+ {\left[\delta([\rho, \nu \rho]) \otimes \sigma+\rho \times \nu \rho \otimes \sigma+\delta\left(\left[\nu^{-1} \rho, \rho\right]\right) \otimes \sigma\right] . }
\end{aligned}
$$

From (5-2) and the above formula for $\mu^{*}(\delta([\rho, \nu \rho]) \rtimes \sigma)$, we can conclude that $\rho \otimes \nu \rho \rtimes \sigma$ is a subquotient of $s_{(p)}(L(\delta([\rho, \nu \rho]), \sigma))$. Further, write

$$
\begin{aligned}
& \mu^{*}(L(\nu \rho, \rho) \rtimes \sigma)=1 \otimes L(\nu \rho, \rho) \rtimes \sigma \\
& \quad+\left[\nu^{-1} \rho \otimes \tau_{1}+\nu^{-1} \rho \otimes \tau_{2}+\rho \otimes \nu \rho \rtimes \sigma\right] \\
& \quad+\left[L(\nu \rho, \rho) \otimes \sigma+\nu^{-1} \rho \times \rho \otimes \sigma+L\left(\rho, \nu^{-1} \rho\right) \otimes \sigma\right] .
\end{aligned}
$$

Now, we claim that $\nu \rho \rtimes \tau_{i}$ has $L(\delta([\rho, \nu \rho]), \sigma)$ as a subquotient. To prove that, it is enough to prove that there exists a nonzero intertwining $\delta([\rho, \nu \rho]) \rtimes \sigma \longrightarrow \nu \rho \rtimes \tau_{i}$. We shall show that now. Consider the composition $\delta([\rho, \nu \rho]) \rtimes \sigma \hookrightarrow \nu \rho \times \rho \rtimes \sigma \stackrel{\mathrm{pr}_{i}}{\longrightarrow}$ $\nu \rho \rtimes \tau_{i}$, where $\operatorname{pr}_{i}$ denotes the projection of $\nu \rho \times \rho \rtimes \sigma$ onto $\nu \rho \rtimes \tau_{i}$ with respect to the decomposition (5-4). Denote it by $\varphi_{i}$. If $\varphi_{i} \neq 0$, then our claim holds. Therefore, suppose that $\varphi_{i}=0$. This implies that there exists an epimorphism of $L(\rho, \nu \rho) \rtimes \sigma \cong(\nu \rho \times \rho \rtimes \sigma) /(\delta([\rho, \nu \rho]) \rtimes \sigma)$ onto $\nu \rho \rtimes \tau_{i}$. This implies there is also an epimorphism on the level of each Jacquet module. Formulas (5-5) and (5-3) imply that this is not possible. This finishes the proof of our claim. 
We can now conclude that $L(\delta([\rho, \nu \rho]), \sigma)$ has multiplicity two in $\nu \rho \times \rho \rtimes \sigma$. Further, it is easy to get that the following equality holds in the Grothendieck group:

$$
L(\nu \rho, \rho) \rtimes \sigma=L\left(\nu \rho, \tau_{1}\right)+L\left(\nu \rho, \tau_{2}\right)+L(\delta([\rho, \nu \rho]), \sigma)
$$

(use (5-1), (5-3) and (5-5) to see that we have the first two summands; the last summand follows from (5-2) and (5-3); the multiplicity one follows from (5-5)).

Note that none of the three irreducible subquotients that we have considered up to now has $\nu \rho \otimes \tau_{i}$ for a subquotient in a suitable Jacquet module (see (5-5)).

Consider $\nu \rho \rtimes \tau_{i}$ and $\delta([\rho, \nu \rho]) \rtimes \sigma$ as subrepresentations in $\nu \rho \times \rho \rtimes \sigma$. Then, from the Jacquet modules, one can conclude that their intersection is nonzero. Moreover, there exists an irreducible subquotient of the intersection which has $\nu \rho \otimes \tau_{i}$ in the suitable Jacquet module. This subquotient must be $\delta\left([\rho, \nu \rho]_{\tau_{i}}, \sigma\right)$ by (ii) of Lemma 3.1 and (i) of Proposition 3.2. Then, $\mu^{*}\left(\delta\left([\rho, \nu \rho]_{\tau_{i}}, \sigma\right)\right)=1 \otimes \delta\left([\rho, \nu \rho]_{\tau_{i}}, \sigma\right)+\nu \rho \otimes$ $\tau_{i}+\delta([\rho, \nu \rho]) \otimes \sigma$. This representation is square integrable by the Casselman's square integrability criterion. The proof of the lemma is now complete.

In the sequel, we shall also use the following notation:

$$
\delta\left([\rho, \rho]_{\tau_{i}}, \sigma\right)=\tau_{i}, \quad \delta\left(\emptyset_{\tau_{i}}, \sigma\right)=\sigma .
$$

5.2. Proposition. Let $m \geq 1$. Then:

(i) $\delta\left(\left[\rho, \nu^{m} \rho\right]_{\tau_{i}}, \sigma\right)$ is square integrable.

(ii) $\mu^{*}\left(\delta\left(\left[\rho, \nu^{m} \rho\right]_{\tau_{i}}, \sigma\right)\right)=\sum_{k=0}^{m+1} \delta\left(\left[\nu^{k} \rho, \nu^{m} \rho\right]\right) \otimes \delta\left(\left[\rho, \nu^{k-1} \rho\right]_{\tau_{i}}, \sigma\right)$.

(iii) We may characterize $\delta\left(\left[\rho, \nu^{m} \rho\right]_{\tau_{i}}, \sigma\right)$ as a unique irreducible subquotient $\pi$ of $\nu^{m} \rho \times \nu^{m-1} \rho \times \cdots \times \nu \rho \rtimes \tau_{i}$ for which $\delta\left(\left[\rho, \nu^{m} \rho\right]\right) \otimes \sigma$ is a subquotient of $s_{(p(m+1))}(\pi)$.

Proof. Since $\mu^{*}\left(\tau_{i}\right)=1 \otimes \tau_{i}+\rho \otimes \sigma$, inductively we get

$$
\begin{aligned}
s_{G L} & \left(\nu^{m} \rho \times \nu^{m-1} \rho \times \cdots \times \nu^{2} \rho \times \nu \rho \rtimes \tau_{i}\right) \\
& =\sum_{\left(\varepsilon_{i}\right) \in\{ \pm 1\}^{m}} \nu^{\varepsilon_{m} m} \rho \times \cdots \times \nu^{\varepsilon_{2} 2} \rho \times \nu^{\varepsilon_{1}} \rho \times \rho \otimes \sigma .
\end{aligned}
$$

From this, one sees that $s_{(p)^{m+1}}\left(\nu^{m} \rho \times \nu^{m-1} \rho \times \cdots \times \nu^{2} \rho \times \nu \rho \rtimes \tau_{i}\right)$ is a multiplicity one representation $\left((p)^{m+1}\right.$ is defined in (1-5)). This implies also that $s_{(p)^{m}}\left(\nu^{m} \rho \times \nu^{m-1} \rho \times \cdots \times \nu^{2} \rho \times \nu \rho \rtimes \tau_{i}\right)$ is a multiplicity one representation. From this one gets that $\nu^{m} \rho \times \nu^{m-1} \rho \times \cdots \times \nu^{2} \rho \times \nu \rho \rtimes \tau_{i}$ has a unique irreducible subrepresentation. Since $\delta\left(\left[\nu \rho, \nu^{m} \rho\right]\right) \rtimes \tau_{i}$ is a subrepresentation of this representation, we see that the irreducible subrepresentation is $\delta\left(\left[\rho, \nu^{m} \rho\right]_{\tau_{i}}, \sigma\right)$. In a similar way one concludes that $\delta\left(\left[\rho, \nu^{m+1} \rho\right]_{\tau_{i}}, \sigma\right)$ is a (unique irreducible) subrepresentation of $\nu^{m+1} \rho \rtimes \delta\left(\left[\rho, \nu^{m} \rho\right]_{\tau_{i}}, \sigma\right), \delta\left(\left[\nu^{m} \rho, \nu^{m+1} \rho\right]\right) \rtimes \delta\left(\left[\rho, \nu^{m-1} \rho\right]_{\tau_{i}}, \sigma\right)$, and generally

$$
\delta\left(\left[\rho, \nu^{m+1} \rho\right]_{\tau_{i}}, \sigma\right) \hookrightarrow \delta\left(\left[\nu^{k+1} \rho, \nu^{m+1} \rho\right]\right) \rtimes \delta\left(\left[\rho, \nu^{k} \rho\right]_{\tau_{i}}, \sigma\right), \quad k=0,1, \ldots, m .
$$

Lemma 5.1 and (ii) of Proposition 3.2 imply that the theorem holds for $m=1$. We proceed by induction now. Suppose that the proposition holds up to $m \geq 1$. Consider $\nu^{m+1} \rho \rtimes \delta\left(\left[\rho, \nu^{m} \rho\right]_{\tau_{i}}, \sigma\right)$. The inductive assumption implies

$$
\begin{aligned}
\text { s.s. }\left(s _ { G L } \left(\nu^{m+1} \rho \rtimes \delta\right.\right. & \left.\left.\left(\left[\rho, \nu^{m} \rho\right]_{\tau_{i}}, \sigma\right)\right)\right) \\
& =\nu^{m+1} \rho \times \delta\left(\left[\rho, \nu^{m} \rho\right]\right) \otimes \sigma+\nu^{-(m+1)} \rho \times \delta\left(\left[\rho, \nu^{m} \rho\right]\right) \otimes \sigma .
\end{aligned}
$$


Further,

$$
\begin{gathered}
\text { s.s. }\left(s_{G L}\left(\delta\left(\left[\nu^{m} \rho, \nu^{m+1} \rho\right]\right) \rtimes \delta\left(\left[\rho, \nu^{m-1} \rho\right]_{\tau_{i}}, \sigma\right)\right)\right) \\
=\delta\left(\left[\nu^{-(m+1)} \rho, \nu^{-m} \rho\right]\right) \times \delta\left(\left[\rho, \nu^{m-1} \rho\right]\right) \otimes \sigma \\
+\nu^{-m} \rho \times \nu^{m+1} \rho \times \delta\left(\left[\rho, \nu^{m-1} \rho\right]\right) \otimes \sigma \\
+\delta\left(\left[\nu^{m} \rho, \nu^{m+1} \rho\right]\right) \times \delta\left(\left[\rho, \nu^{m-1} \rho\right]\right) \otimes \sigma .
\end{gathered}
$$

From this, we see that the two representations under consideration have exactly one irreducible subquotient in common and it has $\delta\left(\left[\rho, \nu^{m+1} \rho\right]\right) \otimes \sigma$ for the corresponding Jacquet module. This common irreducible subquotient is $\delta\left(\left[\rho, \nu^{m+1} \rho\right]_{\tau_{i}}, \sigma\right)$. This implies (i). Claim (ii) follows from $s_{G L}\left(\delta\left(\left[\rho, \nu^{m+1} \rho\right]_{\tau_{i}}, \sigma\right)\right)=\delta\left(\left[\rho, \nu^{m+1} \rho\right]\right) \otimes \sigma,(5-6)$ and the characterization of essentially square integrable representations of general linear groups by Jacquet modules. Since the multiplicity of $\delta\left(\left[\rho, \nu^{m+1} \rho\right]\right) \otimes \sigma$ in $s_{G L}\left(\nu^{m+1} \rho \times \nu^{m} \rho \times \cdots \times \nu \rho \times \tau_{i}\right)$ is one, we get (iii) from $s_{G L}\left(\delta\left(\left[\rho, \nu^{m+1} \rho\right]_{\tau_{i}}, \sigma\right)\right)=$ $\delta\left(\left[\rho, \nu^{m+1} \rho\right]\right) \otimes \sigma$.

5.3. Lemma. Let $\pi$ be an irreducible subquotient of the representation $\left(\prod_{i=-n}^{m} \nu^{i} \rho\right)$ $\rtimes \sigma$ such that $\delta\left(\left[\rho, \nu^{m} \rho\right]\right) \times \delta\left(\left[\nu \rho, \nu^{n} \rho\right]\right) \otimes \sigma \leq s_{G L}(\pi)$. Then

(i) $\pi \leq \delta\left(\left[\nu^{-n} \rho, \nu^{m} \rho\right]\right) \rtimes \sigma$.

(ii) $\delta\left(\left[\nu^{-n} \rho, \nu^{m} \rho\right]\right) \otimes \sigma \leq s_{G L}(\pi)$.

(iii) $\pi \cong \delta\left(\left[\nu^{-n} \rho, \nu^{m} \rho\right]_{\tau_{i}}, \sigma\right)$ for some $i \in\{1,2\}$.

(iv) The multiplicity of $\delta\left(\left[\rho, \nu^{m} \rho\right]\right) \times \delta\left(\left[\nu \rho, \nu^{n} \rho\right]\right) \otimes \sigma$ in $s_{G L}(\pi)$ is one.

Proof. Lemma 3.3 implies (i). Note that $\sum_{i=1}^{2} \delta\left(\left[\nu \rho, \nu^{n} \rho\right]\right) \rtimes \delta\left(\left[\rho, \nu^{m} \rho\right]_{\tau_{i}}, \sigma\right) \leq$ $\left(\prod_{k=-n}^{m} \nu^{k} \rho\right) \rtimes \sigma$ and $\delta\left(\left[\rho, \nu^{m} \rho\right]\right) \times \delta\left(\left[\nu \rho, \nu^{n} \rho\right]\right) \otimes \sigma \leq s_{G L}\left(\delta\left(\left[\nu \rho, \nu^{n} \rho\right]\right) \rtimes \delta\left(\left[\rho, \nu^{m} \rho\right]_{\tau_{i}}, \sigma\right)\right)$ $(i=1,2)$. This and Lemma 3.3 imply

$$
\pi \leq \delta\left(\left[\nu \rho, \nu^{n} \rho\right]\right) \rtimes \delta\left(\left[\rho, \nu^{m} \rho\right]_{\tau_{i_{0}}}, \sigma\right),
$$

for some $i_{0} \in\{1,2\}$. The assumption $\delta\left(\left[\rho, \nu^{m} \rho\right]\right) \times \delta\left(\left[\nu \rho, \nu^{n} \rho\right]\right) \otimes \sigma \leq s_{G L}(\pi)$ implies $\delta\left(\left[\nu \rho, \nu^{m} \rho\right]\right) \times \delta\left(\left[\rho, \nu^{m} \rho\right]\right) \otimes \sigma \leq s_{G L}\left(\delta\left(\left[\nu^{n+1} \rho, \nu^{m} \rho\right]\right) \rtimes \pi\right)$. This and Theorem 2.3 implies $\delta\left(\left[\nu^{-m} \rho, \nu^{m} \rho\right], \sigma\right) \leq \delta\left(\left[\nu^{n+1} \rho, \nu^{m} \rho\right]\right) \rtimes \pi$, which implies, together with (2-11)

$$
\delta\left(\left[\nu^{-m} \rho, \nu^{m} \rho\right]\right) \otimes \sigma \leq s_{G L}\left(\delta\left(\left[\nu^{n+1} \rho, \nu^{m} \rho\right]\right) \rtimes \pi\right) .
$$

Suppose (ii) does not hold. Note that $\delta\left(\left[\nu^{-n} \rho, \nu^{m} \rho\right]\right) \otimes \sigma \leq s_{G L}\left(\delta\left(\left[\nu \rho, \nu^{n} \rho\right]\right) \rtimes\right.$ $\left.\delta\left(\left[\rho, \nu^{m} \rho\right]_{\tau_{i_{0}}}, \sigma\right)\right)$. Similarly, as in the proof of Lemma 4.1, the previous observation, $(5-7),(5-8)$ and the assumption that (ii) does not hold, imply

$$
\begin{aligned}
& \delta\left(\left[\nu^{-m} \rho, \nu^{m} \rho\right]\right) \otimes \sigma \leq \sum_{k=n+1}^{m+1} \delta\left(\left[\nu^{k} \rho, \nu^{m} \rho\right]\right) \times \delta\left(\left[\nu^{-k+1} \rho, \nu^{-n-1} \rho\right]\right) \\
& \times\left(\delta\left(\left[\rho, \nu^{m} \rho\right]\right) \times \sum_{j=1}^{n+1} \delta\left(\left[\nu^{j} \rho, \nu^{n} \rho\right]\right) \times \delta\left(\left[\nu^{-j+1} \rho, \nu^{-1} \rho\right]\right)-\delta\left(\left[\nu^{-n} \rho, \nu^{m} \rho\right]\right)\right) \otimes \sigma,
\end{aligned}
$$

which implies (considering $\nu^{-m} \rho$ and $\nu^{-n} \rho$ )

$$
\begin{aligned}
\delta\left(\left[\nu^{-m} \rho, \nu^{m} \rho\right]\right) \otimes \sigma \leq & \delta\left(\left[\nu^{-m} \rho, \nu^{-n-1} \rho\right]\right) \\
& \times\left(\delta\left(\left[\rho, \nu^{m} \rho\right]\right) \times \delta\left(\left[\nu^{-n} \rho, \nu^{-1} \rho\right]\right)-\delta\left(\left[\nu^{-n} \rho, \nu^{m} \rho\right]\right)\right) \otimes \sigma .
\end{aligned}
$$

Again, in the same way as in the proof of Lemma 4.1 we see that this is not possible. This ends the proof of (ii). 
Further, (i), (ii), (i) of Lemma 3.1 and (i) of Proposition 3.2 imply (iii), while (5-7) implies (iv).

We have proved the following:

5.4. Proposition. Suppose that $\pi$ is an irreducible subquotient of $\left(\prod_{k=-\alpha-n}^{\alpha+m} \nu^{k} \rho\right)$ $\rtimes \sigma$ which satisfies $\delta\left(\left[\rho, \nu^{m} \rho\right]\right) \times \delta\left(\left[\nu \rho, \nu^{n} \rho\right]\right) \otimes \sigma \leq s_{G L}(\pi)$. Then the multiplicity of $\pi$ in $\left(\prod_{k=-\alpha-n}^{\alpha+m} \nu^{k} \rho\right) \rtimes \sigma$ is one, $\pi$ is a subquotient of the representation $\delta\left(\left[\nu^{-n} \rho, \nu^{m} \rho\right]\right) \rtimes \sigma$ and $\pi \cong \delta\left(\left[\nu^{-n} \rho, \nu^{m} \rho\right]_{\tau_{i}}, \sigma\right)$ for some $i \in\{1,2\}$. The multiplicity of $\delta\left(\left[\nu^{-n} \rho, \nu^{m} \rho\right]\right) \otimes \sigma$ and $\delta\left(\left[\rho, \nu^{m} \rho\right]\right) \times \delta\left(\left[\nu \rho, \nu^{n} \rho\right]\right) \otimes \sigma$ in $s_{G L}(\pi)$ is one.

5.5. Theorem. For $0 \leq n<m$ we have

$$
\begin{aligned}
& s_{G L}\left(\delta\left(\left[\nu^{-n} \rho, \nu^{m} \rho\right]_{\tau_{i}}, \sigma\right)\right)=\sum_{k=-n}^{0} \delta\left(\left[\nu^{k} \rho, \nu^{m} \rho\right]\right) \\
& \times \delta\left(\left[\nu^{-k+1} \rho, \nu^{n} \rho\right]\right) \otimes \sigma, \quad i=1,2,
\end{aligned}
$$

and $\delta\left(\left[\nu^{-n} \rho, \nu^{m} \rho\right]_{\tau_{i}}, \sigma\right)$ are square integrable. (The formula holds also if $n=m$.)

Proof. Formula (5-9) and the Casselman's square integrability criterion imply the square integrability of $\delta\left(\left[\nu^{-n} \rho, \nu^{m} \rho\right]_{\tau_{i}}, \sigma\right)$. It remains to prove the formula. If $n=0$, then Proposition 5.2 implies the formula. We shall consider the case $0<n<m$.

First observe $\delta\left(\left[\nu \rho, \nu^{n} \rho\right]\right) \times \delta\left(\left[\rho, \nu^{m} \rho\right]\right) \otimes \sigma \leq s_{G L}\left(\delta\left(\left[\nu \rho, \nu^{n} \rho\right]\right) \rtimes \delta\left(\left[\rho, \nu^{m} \rho\right]_{\tau_{j}}, \sigma\right)\right)$, for $j=1,2$. Now $\sum_{j=1}^{2} \delta\left(\left[\nu \rho, \nu^{n} \rho\right]\right) \rtimes \delta\left(\left[\rho, \nu^{m} \rho\right]_{\tau_{j}}, \sigma\right) \leq\left(\prod_{k=-\alpha-n}^{\alpha+m} \nu^{k} \rho\right) \rtimes \sigma$, Lemma 5.3 and Lemma 3.3 imply $\delta\left(\left[\nu^{-n} \rho, \nu^{m} \rho\right]_{\tau_{i}}, \sigma\right) \leq \delta\left(\left[\nu \rho, \nu^{n} \rho\right]\right) \rtimes \delta\left(\left[\rho, \nu^{m} \rho\right]_{\tau_{j}}, \sigma\right)$ for some $j \in\{1,2\}$. This implies

$$
\begin{aligned}
& s_{G L}(\left.\delta\left(\left[\nu^{-n} \rho, \nu^{m} \rho\right]_{\tau_{i}}, \sigma\right)\right) \leq \delta\left(\left[\rho, \nu^{m} \rho\right]\right) \\
& \quad \times \sum_{k=1}^{n+1} \delta\left(\left[\nu^{k} \rho, \nu^{n} \rho\right]\right) \times \delta\left(\left[\nu^{-k+1} \rho, \nu^{-1} \rho\right]\right) \otimes \sigma .
\end{aligned}
$$

Proposition 3.2 (i) implies

$$
s_{G L}\left(\delta\left(\left[\nu^{-n} \rho, \nu^{m} \rho\right]_{\tau_{i}}, \sigma\right)\right) \leq \sum_{k=-n}^{m+1} \delta\left(\left[\nu^{k} \rho, \nu^{m} \rho\right]\right) \times \delta\left(\left[\nu^{-k+1} \rho, \nu^{n} \rho\right]\right) \otimes \sigma .
$$

Now (5-10) implies that $\nu^{-n-1} \rho$ cannot be in the $G L$-support of irreducible subquotients of $s_{G L}\left(\delta\left(\left[\nu^{-n} \rho, \nu^{m} \rho\right]_{\tau_{i}}, \sigma\right)\right.$. This and (5-11) now imply

$$
s_{G L}\left(\delta\left(\left[\nu^{-n} \rho, \nu^{m} \rho\right]_{\tau_{i}}, \sigma\right)\right) \leq \sum_{k=-n}^{n+1} \delta\left(\left[\nu^{k} \rho, \nu^{m} \rho\right]\right) \times \delta\left(\left[\nu^{-k+1} \rho, \nu^{n} \rho\right]\right) \otimes \sigma .
$$

A term in the above sum is either irreducible, or a multiplicity one representation of length two. Let $\pi=L\left(\delta\left(\Delta_{1}\right), \ldots, \delta\left(\Delta_{l}\right)\right) \otimes \sigma$ be an irreducible subquotient of the representation $s_{G L}\left(\delta\left(\left[\nu^{-n} \rho, \nu^{m} \rho\right]_{\tau_{i}}, \sigma\right)\right)$. Now (5-10) implies that if some $\Delta_{j}$ ends 
with $\nu^{m} \rho$, then $\rho$ must also be in this $\Delta_{j}$. Therefore, we can transform (5-12) into

$$
\begin{aligned}
s_{G L}( & \left.\left(\left[\nu^{-n} \rho, \nu^{m} \rho\right]_{\tau_{i}}, \sigma\right)\right) \\
\leq & \sum_{k=-n}^{0} \delta\left(\left[\nu^{k} \rho, \nu^{m} \rho\right]\right) \times \delta\left(\left[\nu^{-k+1} \rho, \nu^{n} \rho\right]\right) \otimes \sigma \\
& +\sum_{k=1}^{n+1} \delta\left(\left[\nu^{-k+1} \rho, \nu^{m} \rho\right]\right) \times \delta\left(\left[\nu^{k} \rho, \nu^{n} \rho\right]\right) \otimes \sigma \\
= & \sum_{k=-n}^{0} \delta\left(\left[\nu^{k} \rho, \nu^{m} \rho\right]\right) \times \delta\left(\left[\nu^{-k+1} \rho, \nu^{n} \rho\right]\right) \otimes \sigma \\
& +\sum_{k=-n}^{0} \delta\left(\left[\nu^{k} \rho, \nu^{m} \rho\right]\right) \times \delta\left(\left[\nu^{-k+1} \rho, \nu^{n} \rho\right]\right) \otimes \sigma \\
= & 2 \sum_{k=-n}^{0} \delta\left(\left[\nu^{k} \rho, \nu^{m} \rho\right]\right) \times \delta\left(\left[\nu^{-k+1} \rho, \nu^{n} \rho\right]\right) \otimes \sigma .
\end{aligned}
$$

Note that all the terms in the sum on the right-hand side of (5-10) have different $G L$-supports. Now Lemma 7.1 implies that the right-hand side of (5-10) is a multiplicity one representation. Therefore, (5-13) implies that in (5-9) the inequality $\leq$ holds (we could prove this inequality in a different way, similarly as in the proof of Theorem 4.5).

One proves inductively,

$$
\begin{aligned}
& \delta\left(\left[\nu^{-n} \rho, \nu^{m} \rho\right]_{\tau_{i}}, \sigma\right) \hookrightarrow\left(\nu^{m} \rho \times \nu^{m-1} \rho \times \cdots \times \nu \rho \times \rho\right) \\
& \quad \times\left(\nu^{n} \rho \times \nu^{n-1} \rho \times \cdots \times \nu^{-k+1} \rho\right) \times\left(\nu^{-1} \rho \times \nu^{-2} \rho \times \cdots \times \nu^{k} \rho\right) \\
& \rtimes \sigma, k=-n, \ldots,-1,0
\end{aligned}
$$

(see the proof of Lemma 4.4 or (2-6) for a similar proofs; the initial case of induction $i=-n$ follows from (i) of Proposition 3.2).

Observe that all terms in the sum (5-13) are irreducible and have different $G L$ supports. Now, in the same way as in the proof of Lemma 4.4, first applying Frobenius reciprocity to (5-14), and using this in consideration of $G L$-supports of the terms in the sum on the right-hand side of (5-13), we get that the inequality $\geq$ in (5-9) holds. This completes the proof of (5-9).

\section{EXAmple}

It is obvious that the condition that $\delta(\Delta) \rtimes \sigma$ reduces is necessary for the existence of a square integrable subquotient of $\delta(\Delta) \rtimes \sigma$. It is less clear if the condition that $\delta(\Delta \cap \tilde{\Delta}) \rtimes \sigma$ reduces is important for construction of the square integrable representations. The following lemma suggests that this is an important condition.

6.1. Lemma. Let $\rho$ and $\sigma$ be irreducible unitarizable cuspidal representations of $G L(p, F)$ and $S_{q}$, respectively. Suppose that $\nu^{3 / 2} \rho \rtimes \sigma$ reduces and $\nu^{1 / 2} \rho \rtimes \sigma$ is irreducible. Then $\delta\left(\left[\nu^{-1 / 2} \rho, \nu^{3 / 2} \rho\right]\right) \rtimes \sigma$ reduces, $\delta\left(\left[\nu^{-1 / 2} \rho, \nu^{1 / 2} \rho\right]\right) \rtimes \sigma$ is irreducible and $\nu^{-1 / 2} \rho \times \nu^{1 / 2} \rho \times \nu^{3 / 2} \rho \rtimes \sigma$ does not contain any square integrable subquotient.

Proof. The reducibility of $\delta\left(\left[\nu^{-1 / 2} \rho, \nu^{3 / 2} \rho\right]\right) \rtimes \sigma$ follows from the proof of Theorem 13.2 of [T5]. We can conclude this easily from the following facts. First 
$\nu^{1 / 2} \rho \times \delta\left(\left[\nu^{1 / 2} \rho, \nu^{3 / 2} \rho\right]\right) \otimes \sigma$ has multiplicity one in $s_{G L}\left(\nu^{-1 / 2} \rho \times \nu^{1 / 2} \rho \times \nu^{3 / 2} \rho \rtimes \sigma\right)$, $s_{G L}\left(\delta\left(\left[\nu^{-1 / 2} \rho, \nu^{3 / 2} \rho\right]\right) \rtimes \sigma\right)$ and $s_{G L}\left(\delta\left(\left[\nu^{-1 / 2} \rho, \nu^{1 / 2} \rho\right]\right) \rtimes \delta\left(\left[\nu^{3 / 2} \rho, \sigma\right)\right)\right.$. Further, considering $\delta\left(\left[\nu^{-3 / 2} \rho, \nu^{1 / 2} \rho\right]\right) \otimes \sigma$ we get

$$
s_{G L}\left(\delta\left(\left[\nu^{-1 / 2} \rho, \nu^{3 / 2} \rho\right]\right) \rtimes \sigma\right) \not \leq s_{G L}\left(\delta\left(\left[\nu^{-1 / 2} \rho, \nu^{1 / 2} \rho\right]\right) \rtimes \delta\left(\left[\nu^{3 / 2} \rho, \sigma\right)\right) .\right.
$$

The irreducibility of $\delta\left(\left[\nu^{-1 / 2} \rho, \nu^{1 / 2} \rho\right]\right) \rtimes \sigma$ follows from the proof Proposition 4.1 of [T5] (in that proof the irreducibility of $\nu^{1 / 2+k} \rho \rtimes \sigma$ for integral $k>1$ is not used). One can also prove this irreducibility easily directly from the facts s.s. $\left(s_{(p)}\left(\delta\left(\left[\nu^{-1 / 2} \rho, \nu^{1 / 2} \rho\right]\right) \rtimes \sigma\right)\right)=2 \nu^{1 / 2} \rho \otimes \nu^{1 / 2} \rho \rtimes \sigma$ and $\nu^{1 / 2} \rho \times \nu^{1 / 2} \rho \otimes \sigma \leq$ $s_{G L}\left(\delta\left(\left[\nu^{-1 / 2} \rho, \nu^{1 / 2} \rho\right]\right) \rtimes \sigma\right)$.

Let $\pi$ be an irreducible square integrable subquotient of $\nu^{-1 / 2} \rho \times \nu^{1 / 2} \rho \times \nu^{3 / 2} \rho \rtimes \sigma$. Let $\nu^{\epsilon_{1}} \rho \otimes \nu^{\epsilon_{2}} \rho \otimes \nu^{\epsilon_{3}} \rho \otimes \sigma$ be any irreducible subquotient of $s_{(p, p, p)}(\pi)$. Then one can find $s_{1}, s_{2}, s_{3} \in\{ \pm 1\}$ such that $\epsilon_{1}, \epsilon_{2}, \epsilon_{3}$ equals $s_{1} 1 / 2, s_{2} 1 / 2, s_{3} 3 / 2$ up to the word ordering. Now:

(1) The Casselman's square integrability criterion implies $s_{3}=1$ and $\epsilon_{1}>0$.

(2) We have s.s. $\left(s_{G L}\left(\delta\left(\left[\nu^{-1 / 2} \rho, \nu^{1 / 2} \rho\right]\right) \rtimes \delta\left(\nu^{3 / 2} \rho, \sigma\right)\right)\right)=2 \delta\left(\left[\nu^{-1 / 2} \rho, \nu^{1 / 2} \rho\right]\right) \times$ $\nu^{3 / 2} \rho \otimes \sigma+\nu^{1 / 2} \rho \times \nu^{1 / 2} \rho \times \nu^{3 / 2} \rho \otimes \sigma$. Further, each irreducible subquotient of $\delta\left(\left[\nu^{-1 / 2} \rho, \nu^{1 / 2} \rho\right]\right) \rtimes \delta\left(\nu^{3 / 2} \rho, \sigma\right)$ is tempered, but it is not square integrable.

(3) Note s.s. $\left(s_{G L}\left(\nu^{-1 / 2} \rho \times \nu^{1 / 2} \rho \times \nu^{3 / 2} \rho \rtimes \sigma\right)\right)-$ s.s. $\left(s_{G L}\left(\delta\left(\left[\nu^{-1 / 2} \rho, \nu^{1 / 2} \rho\right]\right) \rtimes\right.\right.$ $\left.\left.\delta\left(\nu^{3 / 2} \rho, \sigma\right)\right)\right) \leq \sum_{\left(k_{1}, k_{2}, k_{3}\right) \in\{ \pm 1\}^{3} \backslash(1,1,1)} \nu^{k_{1} 1 / 2} \rho \times \nu^{k_{2} 1 / 2} \rho \times \nu^{k_{3} 3 / 2} \rho \otimes \sigma$ (use the formula in the proof of Lemma 3.3 for s.s. $\left(s_{G L}\left(\nu^{-1 / 2} \rho \times \nu^{1 / 2} \rho \times \nu^{3 / 2} \rho \rtimes \sigma\right)\right)$, and $\left.\nu^{1 / 2} \rho \times \nu^{1 / 2} \rho \times \nu^{3 / 2} \rho \otimes \sigma \leq s_{G L}\left(\delta\left(\left[\nu^{-1 / 2} \rho, \nu^{1 / 2} \rho\right]\right) \rtimes \delta\left(\nu^{3 / 2} \rho, \sigma\right)\right)\right)$.

(4) Suppose $\epsilon_{1}, \epsilon_{2}, \epsilon_{3}$ are all positive. Then (3) implies that $\pi$ is a subquotient of the representation $\delta\left(\left[\nu^{-1 / 2} \rho, \nu^{1 / 2} \rho\right]\right) \rtimes \delta\left(\nu^{3 / 2} \rho, \sigma\right)$. This contradicts the square integrability of $\pi$ (see (2)). Thus, at least one $\epsilon_{i}$ is negative.

(5) Let us choose an irreducible subquotient $\nu^{\epsilon_{1}} \rho \otimes \nu^{\epsilon_{2}} \rho \otimes \nu^{\epsilon_{3}} \rho \otimes \sigma$ of $s_{(p, p, p)}(\pi)$ such that $\pi \hookrightarrow \nu^{\epsilon_{1}} \rho \times \nu^{\epsilon_{2}} \rho \times \nu^{\epsilon_{3}} \rho \rtimes \sigma$ (we can do it by Corollary 7.2.2 of [C1]). Conversely, if $\pi \hookrightarrow \nu^{\epsilon_{1}^{\prime}} \rho \times \nu^{\epsilon_{2}^{\prime}} \rho \times \nu^{\epsilon_{3}^{\prime}} \rho \rtimes \sigma$, then Frobenius reciprocity implies that $\nu^{\epsilon_{1}^{\prime}} \rho \otimes \nu^{\epsilon_{2}^{\prime}} \rho \otimes \nu^{\epsilon_{3}^{\prime}} \rho \otimes \sigma$ is a quotient of $s_{(p, p, p)}(\pi)$. Now (1) implies that we have the following three possibilities:

(6) $\epsilon_{3}=3 / 2$ and $\epsilon_{1}=1 / 2$. Now (4) implies $\epsilon_{2}=-1 / 2$. Applying Frobenius reciprocity, we get that this contradicts the Casselman's square integrability criterion, since $\pi$ was supposed to be square integrable.

(7) $\epsilon_{2}=3 / 2$ and $\epsilon_{1}=1 / 2$. Again (4) implies $\epsilon_{3}=-1 / 2$. Thus $\pi \hookrightarrow \nu^{1 / 2} \rho \times$ $\nu^{3 / 2} \rho \times \nu^{-1 / 2} \rho \rtimes \sigma \cong \nu^{1 / 2} \rho \times \nu^{3 / 2} \rho \times \nu^{1 / 2} \rho \rtimes \sigma$, which contradicts (4).

(8) $\epsilon_{1}=3 / 2$. Now $\nu^{\epsilon_{1}} \rho \times \nu^{\epsilon_{2}} \rho \times \nu^{\epsilon_{3}} \rho \rtimes \sigma \cong \nu^{\epsilon_{1}} \rho \times \nu^{\epsilon_{2}} \rho \times \nu^{-\epsilon_{3}} \rho \rtimes \sigma$. This and (4) imply $\epsilon_{2}=-1 / 2$. Thus $\pi \hookrightarrow \nu^{3 / 2} \rho \times \nu^{-1 / 2} \rho \times \nu^{\epsilon_{3}} \rho \rtimes \sigma \cong \nu^{-1 / 2} \rho \times \nu^{3 / 2} \rho \times$ $\nu^{\epsilon_{3}} \rho \rtimes \sigma$, which contradicts to (1).

The proof is now complete.

\section{Appendix}

In this section we shall prove one very simple (and probably very well known) fact about representations of general linear groups, for which we do not know a suitable reference.

7.1. Lemma. Let $\Delta_{1}, \ldots, \Delta_{n}$ be segments in irreducible cuspidal representations of general linear groups. Suppose $n \leq 3$. Then $\delta\left(\Delta_{1}\right) \times \cdots \times \delta\left(\Delta_{n}\right)$ is a multiplicity one representation. 
Proof. Using either Theorem 2.3 and Corollary 3.9 of $[\mathrm{A}]$, or the fifth section of [ScSt], one directly gets that for the lemma it is enough to prove that

$$
\left\langle\Delta_{1}\right\rangle \times \cdots \times\left\langle\Delta_{n}\right\rangle \text { is a multiplicity one representation, }
$$

where $\langle\Delta\rangle$ denotes the representations introduced in [Z]. In the rest of this proof we shall use freely the notation introduced in [Z].

Let $X$ be the set of all multisegments $a$ such that $a \leq\left(\Delta_{1}, \Delta_{2}, \ldots, \Delta_{n}\right)$.

(1) It is well known that if $a$ is minimal or maximal in $X$, then the multiplicity of $\langle a\rangle$ is one (see Section 1$)$. Therefore, $(7-1)$ holds if card $(X) \leq 2$. This implies that (7-1) holds if $n \leq 2$.

In the rest of the proof we assume $n=3$.

(2) If two of the segments coincide, then Card $(X) \leq 2$, and (7-1) holds by (1).

(3) Suppose that each $\Delta_{i}$ consists of a single (irreducible cuspidal) representation. Then (7-1) holds (if these cuspidal representations are different, then $\left\langle\Delta_{1}\right\rangle \times$ $\cdots \times\left\langle\Delta_{3}\right\rangle$ is a regular representation; otherwise (2) implies (7-1)).

Now we shall proceed by induction on the total number $k$ of the representations in the support of $\left\langle\Delta_{1}\right\rangle \times \cdots \times\left\langle\Delta_{3}\right\rangle$ (counted with multiplicities). Clearly, $k \geq 3$. For $k=3$ the lemma holds by (3).

Let $k>3$ and suppose that $(7-1)$ holds for $k^{\prime}<k$. Recall that the derivative is

$$
\mathcal{D}\left(\prod_{i=1}^{3}\left\langle\Delta_{i}\right\rangle\right)=\prod_{i=1}^{3}\left(\left\langle\Delta_{i}\right\rangle+\left\langle\Delta_{i}^{-}\right\rangle\right) .
$$

(4) If all three segments have different ends, then looking at the multiplicity of the highest derivatives of any irreducible subquotient, we get from the inductive assumption that the highest derivative has multiplicity one in the derivative (since the assumption on the ends and the inductive assumption imply that the multiplicity of any irreducible representation of a general linear group in $\prod_{i=1}^{3}\left(\left\langle\Delta_{i}\right\rangle+\left\langle\Delta_{i}^{-}\right\rangle\right)-\prod_{i=1}^{3}\left\langle\Delta_{i}\right\rangle$ is either 0 or 1$)$. This implies (7-1).

(5) If all three segments have different beginnings, then passing to contragredients, we get different ends. From (4) we now easily get that (7-1) holds in this situation.

(6) If $\Delta_{1} \cap \Delta_{2} \cap \Delta_{3} \neq \emptyset$, then considering the highest derivatives of the irreducible subquotients, the inductive assumption implies the multiplicity one of all irreducible subquotients (since then all the highest derivatives of the irreducible subquotients are subquotients of $\prod_{i=1}^{3}\left\langle\Delta_{i}^{-}\right\rangle$, and this is a multiplicity one representation by the inductive assumption). Thus, (7-1) holds.

(7) If all three segments have the same beginnings, or the same ends, then $\operatorname{card}(X)=1$, and (7-1) holds by $(1)$.

It remains to consider the case of different segments, when exactly two of the segments have the same beginnings, exactly two of them have the same ends, their intersection is empty and $\operatorname{card}(X)>1$. One easily sees that for such segments, there exists an irreducible cuspidal representation $\rho$ of some $G L(p, F), a, b \in \mathbb{Z}$ satisfying $0 \leq a<b$, such that the segments $\Delta_{i}$ are segments $\left[\rho, \nu^{b} \rho\right],\left[\rho, \nu^{a} \rho\right]$ and $\left[\nu^{a+1} \rho, \nu^{b} \rho\right]$ up to an order. Then Card $(X)=2$, and therefore (7-1) holds by (1). This ends the proof of (7-1), and the proof of the lemma is now complete. 


\section{REFERENCES}

[A] A.-M. Aubert, Dualité dans le groupe de Grothendieck de la catégorie des représentations lisses de longueur finie d'un groupe réductif p-adique, Trans. Amer. Math. Soc. 347 (1995), 2179-2189 (and Erratum, Trans. Amer. Math. Soc. 348 (1996), 4687-4690). MR 95i:22025; MR 97c:22019

[C1] W. Casselman, Introduction to the theory of admissible representations of $p$-adic reductive groups, preprint.

[C2] - The unramified principal series of p-adic groups I. The spherical function, Compositio Math. 40 (3) (1980), 387-406. MR 83a:22018

[GP] B.H. Gross and D. Prasad, On the decomposition of a representation of $\mathrm{SO}_{n}$ when restricted to $\mathrm{SO}_{n-1}$, Canad. J. Math. 44 (5) (1992), 974-1002. MR 93j:22031

[KL] D. Kazhdan and G. Lusztig, Proof of the Deligne-Langlands conjecture for Hecke algebras, Invent. Math. 87 (1987), 153-215. MR 88d:11121

[Moe] C. Møglin, Letter, February 1997.

[Mi1] G. Muić, Some results on square integrable representations; Irreducibility of standard representations, International Mathematics Research Notes 14 (1998), 705-726. MR 99f:22031

[Mi2] - On generic irreducible representations of $S p(n, F)$ and $S O(2 n+1, F)$, Glasnik Mat. 33(53) (1998), 19-31. CMP 99:03

[MrRp] F. Murnaghan, and J. Repka, Reducibility of some induced representations of split classical p-adic groups, Compositio Math. 114 (1998), 263-313. CMP 99:06

[Ree] M. Reeder, Hecke algebras and harmonic analysis on p-adic groups, Amer. J. Math. 119 (1997), 225-248. MR 99c:22025

[Ro] F. Rodier, Sur les représentations non ramifiées des groupes réductifs p-adiques; l'example de GSp(4), Bull. Soc. Math. France 116 (1988), 15-42. MR 89i:22033

[ScSt] P. Schneider and U. Stuhler, Representation theory and sheaves on the Bruhat-Tits building, Inst. Hautes Itudes Sci. Publ. Math. (1997), 97-191. MR 98m:22023

[Sh1] F. Shahidi, A proof of Langlands conjecture on Plancherel measures; complementary series for p-adic groups, Ann. of Math. 132 (1990), 273-330. MR 91m:11095

[Sh2] - Twisted endoscopy and reducibility of induced representations for $p$-adic groups, Duke Math. J. 66 (1992), 1-41. MR 93b:22034

[Si] A. Silberger, Discrete series and classifications for p-adic groups I, Amer. J. Math. 103 (1981), 1231-1321. MR 83j:22011

[T1] M. Tadić, Induced representations of $G L(n, A)$ for $p$-adic division algebras $A$, J. Reine Angew. Math. 405 (1990), 48-77. MR 91i:22025

[T2] L Representations of p-adic symplectic groups, Compositio Math. 90 (1994), 123-181. MR 95a:22025

[T3] _ Structure arising from induction and Jacquet modules of representations of classical p-adic groups, Journal of Algebra 177 (1) (1995), 1-33. MR 97b:22023

[T4] $\quad$ On regular square integrable representations of p-adic groups, Amer. J. Math. 120 (1) (1998), 159-210. CMP 98:07

[T5] — On reducibility of parabolic induction, Israel J. Math. 107 (1998), 29-91. CMP 99:05

[T6] _ A family of square integrable representations of classical p-adic groups, preprint (1998).

[V] D.A. Vogan, The local Langlands conjecture, Contemp. Math. 145 (1993), 305-379. MR 94e:22031

[Z] A.V. Zelevinsky, Induced representations of reductive p-adic groups. II, On irreducible representations of $G L(n)$, Ann. Sci École Norm Sup. 13 (1980), 165-210. MR 83g:22012

Department of Mathematics, University of Zagreb, Bijenička 30, 10000 Zagreb, Croatia

E-mail address: tadic@math.hr 